\title{
ROYAL ACADEMY OF \\ MEDICINE IN IRELAND
}

\section{IRISH JOURNAL OF MEDICAL SCIENCE}

\author{
Sylvester O'Halloran Meeting
}

5th and 6th March 2010

Jean Monnet Theatre

DG016, Main Building

University of Limerick

Irish Journal of Medical Science

Volume 179 Supplement 1

DOI 10.1007/s11845-010-0470-x

算 Springer 
These abstracts are published exactly as received from submitting authors. The opinion and views expressed are those of the authors and have not been verified by the publishers or the editors, who accept no scientific responsibility for the statement made or for the accuracy of the data presented. Any typing or other errors are the author's own.

(c) Royal Academy of Medicine in Ireland 2010.

Published by Springer-Verlag London Ltd, 236 Gray’s Inn Road, 6th Floor, London WC1X 8HL, UK.

Tel: +44(0)203 1922762 email: ruth.cranks@ springer.com 


\section{Sponsors of the Sylvester O'Halloran Meeting 2010}

\section{LEO Pharma}

sanofi aventis

Because health matters

Bayer Healthcare

Bayer Schering Pharma

astellas

Leading Light for Life
(Sponsors of O'Halloran Prize for best Oral Paper)

sanofi-aventis

(Sponsors of Prize for best Poster)

Bayer Healthcare

(Sponsors of Orthopaedic Prizes)

Astellas Pharma Co., Ltd.

(Sponsors of Anaesthesia Prize)

The Sylvester O'Halloran Meeting will sponsor the following:

The Prize for best Head \& Neck Paper

HSE

Feidhmeannacht na Seirbhise Slainte

Health Service Executive

HC21 healthcare

Pfizer

AstraZeneca

MED surgical

Johnson \& Johnson

IPSEN

Innovation for patient care

Tekno Surgical

Sisk Group
Mid-Western Regional Hospital, Limerick

HC Healthcare 21

Medical \& Surgical Suppliers

Pfizer

AstraZeneca Pharmaceuticals

Med Surgical

Johnson \& Johnson

Ipsen Pharmaceuticals

Tekno Surgical Ltd. 


\section{Sponsors of the Sylvester O'Halloran Meeting 2010}

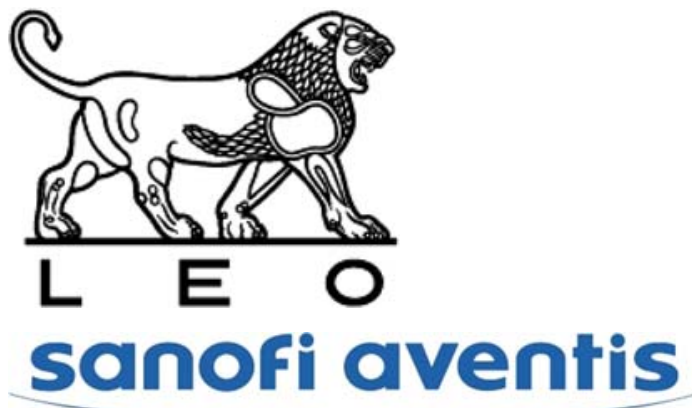

Because health matters

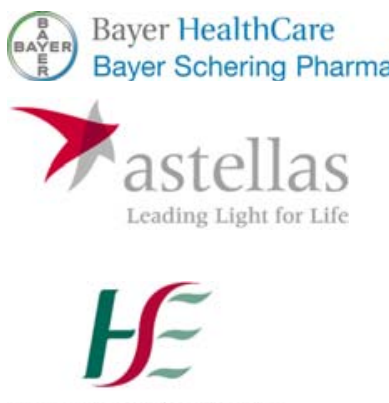

Feidhmeannacht na Seirbhíse Sláinte Health Service Executive
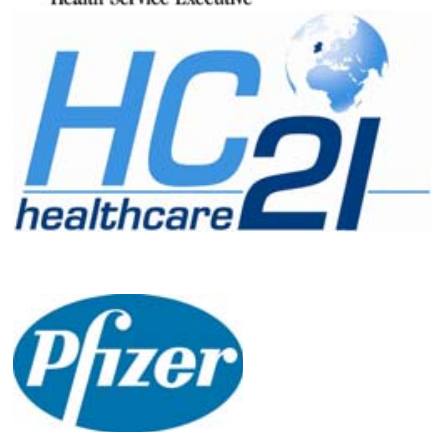

AstraZeneca 2

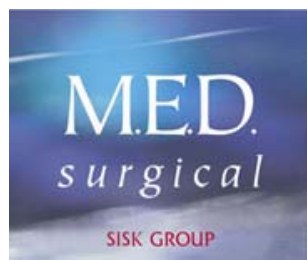

Gohnson affohuson MEDICAL

\section{S. IPSEN}

\section{TEKNO SURGICAL}

SISK GROUP
LEO Pharma (Sponsors of Prize for best Poster)

sanofi-aventis (Sponsors of Prize for best Poster)

Bayer Healthcare (Sponsors of Orthopaedic Prizes)

Astellas Pharma Co., Ltd. (Sponsors of Anaesthesia Prize)

Mid-Western Regional Hospital, Limerick

HC Healthcare 21Medical, Surgical Suppliers

Pfizer

AstraZeneca Pharmaceuticals

Med Surgical

Johnson \& Johnson

Ipsen Pharmaceuticals

Tekno Surgical Ltd. 


\section{Sylvester O'Halloran Meeting 5th and 6th March 2010}

\section{Draft Programme}

Friday 05/03/10 Session 1 Scientific $(2.00 \mathrm{pm})$

Time Allowed: 7 min Speaking

3 min Discussion

Jean Monnet Theatre DG016, Main Building University of Limerick

Chairpersons: Mr. Eoghan Condon \& Mr. Ciaran McDonnell

Session 1: 14.00-15.50

14.00-14.10 1. Faecal incontinence: where is the sense in it all? C. Peirce ${ }^{1}$, C. O'Herlihy ${ }^{1,2}$, P.R. O'Connell ${ }^{1,3}$, J.F.X. Jones ${ }^{1}$ (School of Medicine and Medical Science, University College Dublin, Dublin 4, Ireland ${ }^{1}$, National Maternity Hospital, Holles Street, Dublin, Ireland ${ }^{2}$, Professorial Surgical Unit, St. Vincent's University Hospital, Dublin 4, Ireland ${ }^{3}$ )

14.10-14.20 2. A systemic miRNA signature predictive of early breast cancer

H.M. Heneghan, N.Miller, R. Kelly, M. Nugent, M.J. Kerin (Department of Surgery, National University of Ireland, Galway, Ireland)

14.20-14.30 3 Benefit of adjuvant chemotherapy in luminal A breast cancers A. McDermott ${ }^{1}$, H.M. Heneghan ${ }^{1}$, C. Malone ${ }^{1}$, R. McLaughlin ${ }^{1}$, K.J. Sweeney ${ }^{2}$, M.J. Kerin ${ }^{1}$ (Department of Surgery, Galway University Hospital ${ }^{1}$ National Breast Cancer Screening Programme, University College Hospital, Galway, Ireland ${ }^{2}$ )

14.30-14.40 4. Hypoxia inhibits colonic ion transport through activation of AMP Kinase D. Collins ${ }^{1,2,3}$, S. Kopic ${ }^{3}$, J. Bachlechner ${ }^{3}$, A.M. Hogan ${ }^{1,2}$, M. Medani ${ }^{1,2}$, R. Kennelly ${ }^{1,2}$, J.P. Geibel ${ }^{3}$, D.C. Winter ${ }^{1}$ (Institute for Clinical Outcomes Research and Education (iCORE), St Vincent's University Hospital, Dublin, Ireland ${ }^{1}$, College of Life Sciences and Conway Institute, UCD, Dublin, Ireland ${ }^{2}$, Department of Surgery, Yale University, New Haven, CT, USA ${ }^{3}$ )

14.40-14.50 5. Junctional adhesion molecule-A (JAM-A): a potential target for breast cancer anti-proliferative therapy? G.O. Offiah, E.A. McSherry, A.D.K. Hill, A.M. Hopkins (Department of Surgery, Royal College of Surgeons in Ireland, Dublin 9, Ireland)

14.50-15.00 6. Bacterial superantigens enhance the in vitro pro-inflammatory cytokine release and in vivo lethality of the TLR2 agonist bacterial lipoprotein

D.E. Kearney, J.H. Wang, H.P. Redmond (Department of Academic Surgery, Cork, Ireland University Hospital, Cork, Ireland)

15.00-15.10 7. Unravelling a novel role of myeloid-related proteins 8/14 (Mrp 8/14) in self-tolerance and cross-tolerance to gramnegative and gram-positive bacteria

A.P. Coveney, J.H. Wang, H.P. Redmond (Department of Academic Surgery, Cork University Hospital, Wilton, Cork, Ireland)

15.10-15.20 8. Urinary bladder matrix tubular scaffolds as a tissue engineered vascular graft

A. Callanan, M.T. Walsh, T.M. McGloughlin (Centre for Applied Biomedical Engineering Research (CABER), Department of Mechanical and Aeronautical Engineering, Materials and Surface Science Institute (MSSI), University of Limerick, Ireland)

15.20-15.30 9. Are urinary bladder volumes maintained using xenogenic urinary bladder matrix (UBM) grafts in ovine partial cystectomy models?

N.F. Davis ${ }^{1,2}$ A. Callanan ${ }^{2}$, B.B. McGuire ${ }^{1}$, H.D. Flood ${ }^{1}$, T.M. McGloughlin ${ }^{2}$ (Department of Urology, Mid-Western Regional HospitaL, Limerick, Ireland ${ }^{1}$, The Centre for Applied Biomedical Engineering Research, University of Limerick, Castletroy, Co. Limerick, Ireland ${ }^{2}$ )

15.30-15.40 10. Strength of atherosclerotic plaque in carotid artery

M. Lawlor ${ }^{1}$, E. Kavanagh ${ }^{2}$, P.A. Grace ${ }^{2}$, M.Walsh ${ }^{1}$ (Centre for Applied Biomedical Engineering Research, University of Limerick, Ireland ${ }^{1}$, Department of Vascular Surgery, Mid-Western Regional Hospital, Limerick, Ireland ${ }^{2}$ )

15.40-15.50 11. Overexpression of IRAK-1 prevents BLP-induced tolerance bypromoting NF-kB p65 nuclear transactivation J.H. Liu, C.H. Li, H.P. Redmond, J.H. Wang (Department of Academic Surgery, Cork University Hospital, Cork, Ireland)

15.50-16.10 Coffee

Visit Posters \& Trade Exhibition 


\section{Friday 5/03/10 Session 2 Prize Session-Sponsored By Leo Pharma (4.10 pm)}

Time Allowed: 7 min Speaking

3 min Discussion

Chairpersons: Ms. Shona Tormey \& Mr. Daragh Moneley

Session 2: 16.10-18.00

16.10-16.20 12. Prognostic Signature in Pancreas Cancer

M.J.F. Smith, A. Rogers, J.F. Murphy, A. McDermott, N. Swan, P. Crotty, P.F. Ridgway, K.C. Conlon (The Professorial Surgical Unit, Trinity College Dublin, Ireland, The Trinity Centre for Health Sciences, AMNCH, Tallaght, Dublin 24, Ireland)

16.20-16.30 13. ACE up the sleeve-are vascular patients medically optimized?

A.P. Coveney, E.M. Quinn, P. Cronin, G.C. O’Brien, G. Fulton (Departments of Academic and Vascular Surgery, Cork University Hospital, Wilton, Cork, Ireland)

16.30-16.40 14. Neo-adjuvant therapy for rectal cancer: examining the pathological response E. O’Halloran, A.M. Hogan, F.Elgillani, K.J. Breen, K. Mealy (Department of Surgery, Wexford General Hospital)

16.40-16.50 15. Experimental rupture of realistic abdominal aortic aneurysms: can rupture locations be predicted numerically? B.J. Doyle, A.J. Cloonan, M.T. Walsh, T.M. McGloughlin (Centre for Applied Biomedical Engineering Research (CABER), Department of Mechanical and Aeronautical Engineering, and Materials and Surface Science Institute, University of Limerick, Ireland)

16.50-17.00 16. Pre-clinical evaluation of the Prolong® vascular graft

G.T. Carroll ${ }^{1}$, T.M. McGloughlin ${ }^{1}$, P.A. Grace ${ }^{2}$, M.T. Walsh ${ }^{1}$ (Centre for Applied Biomedical Engineering Research (CABER), Department of Mechanical and Aeronautical Engineering, and the Materials and Surface Science Institute, University of Limerick, Limerick, Ireland ${ }^{1}$, Department of Vascular Surgery, HSE Mid-Western Regional Hospital, Limerick, Ireland ${ }^{2}$ )

17.00-17.10 17. Xenogenic extracellular matrices as potential biomaterials for functional reconstruction of the lower genitourinary tracts

N.F. Davis ${ }^{1,2}$ A. Callanan ${ }^{2}$, B.B. McGuire ${ }^{1}$, H.D. Flood ${ }^{1}$, T.M. McGloughlin ${ }^{2}$ (Department of Urology, Mid-Western Regional Hospital, Limerick, Ireland ${ }^{1}$, The Centre for Applied Biomedical Engineering Research, University of Limerick, Castletroy, Co. Limerick, Ireland ${ }^{2}$ )

17.10-17.20 18. Expression of microRNAs and their predicted protein targets in the circulation of breast cancer patients: novel biomarkers for breast cancer

R.J. Kelly, H.M. Heneghan, N. Miller, M.J. Kerin (Department of Surgery, Clinical Science Institute, National University of Ireland, Galway, Ireland)

17.20-17.30 19. A SNP in let-7 miRNA binding site in the KRAS oncogene increases risk of triple negative breast cancer H.M. Heneghan ${ }^{1}$, N. Miller ${ }^{1}$, T. Paranjape ${ }^{3}$, F.J. Slack ${ }^{2}$, J.B. Weidhaas ${ }^{3}$, M.J. Kerin ${ }^{1}$ (Department of Surgery, National University of Ireland, Galway, Ireland ${ }^{1}$, Department of Molecular, Cellular and Developmental Biology, Yale University, New Haven Connecticut ${ }^{2}$ Department of Therapeutic Radiology, Yale University, New Haven Connecticut ${ }^{3}$ )

17.30-17.40 20. The adipocytokine Leptin and its pathway are up regulated in obese breast cancer patients: a pathway for obesity in breast cancer

P.A. Carroll ${ }^{1}$, L. Healy ${ }^{2}$, E. Allot $^{1}$, J. Lysaght ${ }^{1}$, B. Dunne ${ }^{3}$, M. Griffin ${ }^{3}$, T. Boyle ${ }^{1}$, J.V. Reynolds ${ }^{1}$, M.J. Kennedy ${ }^{4}$, G. Pidgeon $^{1}$, E.M. Connolly ${ }^{1}$ (Department of Surgery, St. James's Hospital and Trinity College Dublin, Ireland ${ }^{1}$, Department of Clinical Nutrition, St. James's Hospital, Dublin, Ireland ${ }^{2}$, Department of Pathology, St. James's Hospital, Dublin, Ireland ${ }^{3}$, Academic Unit of Clinical and Molecular Oncology, St. James's Hospital and Trinity College Dublin, Ireland ${ }^{4}$ )

17.40-17.50 21. Molecular classification of breast cancer and relationship to survival M.C. Hartmann, A.M. Kiely, M.C. Whelan, C. Curran, M.J. Kerin (Department of Surgery, National University of Ireland Galway, Galway, Ireland)

17.50-18.00 22. Columnar cell lesions of the breast: a 5 years experience of a breast unit A. Calamai ${ }^{4}$, R. Salman ${ }^{3}$, M. Kennedy ${ }^{1}$, F. Flanagan ${ }^{2}$, T. Gorey ${ }^{3}$, M. Kell ${ }^{3}$, M. Stokes ${ }^{3}$ (Department of Histopathology ${ }^{1}$, Radiology ${ }^{2}$ and Surgery ${ }^{3}$ and Breast Check and Breast Health, Mater Misericordiae University Hospital, University College Dublin $^{4}$, Dublin, Ireland) 
18.00-18.20 - Visit Posters \& Trade Exhibition

18.20-19.10

\begin{tabular}{|c|}
\hline $18^{\text {th }}$ Sylvester O'Halloran Lecture \\
"Cholecystectomy - outcomes and \\
lessons from history" \\
Presented By: \\
"Professor James Garden"
\end{tabular}

Friday 05/03/10 Session 3 Head and Neck Session (16.45 pm)

Venue: Wood Room - The White House, University of Limerick

Time Allowed: 7 min Speaking

3 min Discussion

Chairpersons: Mr. Neville Shine \& Mr. Alwyn D'Souza

Session 3: 16.45-18.35

16.45-16.55 23. The use of lymphocyte to white cell count ratio in the diagnosis of infectious mononucleosis

P. Lennon, J.P. O'Neill, J. Fenton, T.P. O'Dwyer (Department of Otolaryngology, Mater Misericordiae University Hospital, Eccles St., Dublin 7, Ireland)

16.55-1705 24. Total thyroidectomy: prediction of hypocalcaemia by post-operative parathormone measurement D. Fitzgerald, C.Timon (Royal Victoria Eye and Ear Hospital, Adelaide Road, Dublin 2, Ireland)

17.05-17.15 25. Finite element analysis model development for an edentulous atrophic mandible: the bucket handle fracture E.P. Kavanagh ${ }^{1}$, F. Wallis ${ }^{2}$, T. McGloughlin ${ }^{1}$ G. Kearns ${ }^{3}$, J. Jarvis ${ }^{4}$ (Centre for Applied Biomedical Engineering Research (CABER), University of Limerick, Ireland ${ }^{1}$, Department of Radiology, Mid-Western Regional Hospital, Limerick, Ireland ${ }^{2}$, National Maxillofacial Unit, St. James Hospital, Dublin, Ireland ${ }^{3}$, Department of Mechanical and Aeronautical Engineering, University of Limerick, Ireland ${ }^{4}$ )

17.15-17.25 26. Is there a correlation between intraoperative hypothermia and postoperative morbidity in head and neck cancer patients?

R. Mehanna, J.P. O’Neill, C. Timon (Otorhinolaryngology Head and Neck Surgery Department St. James Hospital, Dublin 8, Ireland)

17.25-17.35 27. Investigating the effect of tonsillectomy on voice C.B. Heffernan, M.A. Rafferty (The Royal Victoria Eye and Ear Hospital, Adelaide Road, Dublin 2, Ireland)

17.35-17.45 28. A prospective trial of pain after minimally invasive video assisted versus minimally invasive open thyroidectomy J.P. O’Neill, C. Timon (Department of Otolaryngology, Head and Neck Surgery, St James Hospital, Dublin, Ireland)

17.45-17.55 29. Management of parotid stone calculus

D. Galvin, A. Curran (Royal Victoria Eye and Ear Hospital, Adelaide Road, Dublin 2, Ireland)

17.55-18.05 30. Complications of cochlear implant surgery: Beaumont Hospital, Dublin 1995-2008

D.R. Gough, A. O'Connor, P. Walshe, L. Viani (National Cochlear Implant Centre, Beaumont Hospital, Dublin, Ireland)

18.05-18.15 31. Electronic follow up of laceration in the emergency department

T.W. M. Walker ${ }^{1}$, S. Byrne ${ }^{2}$, P.J. McCann ${ }^{3}$ (Department of Oral and Maxillofacial Surgery, Mid-Western Regional Hospital, Limerick, Ireland ${ }^{1}$, National University of Ireland Galway, Ireland ${ }^{2}$, Department of Oral and Maxillofacial Surgery, University Hospital, Galway, Ireland ${ }^{3}$ )

18.15-18.25 32. And the winner is..-a scoring system for grading surgical oral presentations B.G. Fennessy, J. Saunders, J.E. Fenton (Department of ENT, Mid-Western Regional Hospital, Limerick, Ireland)

18.25-18.35 33. Functional surgery using conscious sedation for temporomandibular joint eminectomy procedures: a case series M. O’Halloran, L.F.A. Stassen (National Maxillofacial Unit, St. James's Hospital, Dublin 8, Ireland) 
18.00-18.20 - Visit Posters \& Trade Exhibition

18.20-19.10

\begin{tabular}{c}
\hline $1^{\text {th }}$ Sylvester O'Halloran Lecture \\
"Cholecystectomy - outcomes and \\
lessons from history" \\
Presented By: \\
"Professor James Garden"
\end{tabular}

20.30 Reception (Dunraven Arms Hotel, Adare, Co. Limerick)

21.00 Dinner (Dress Informal)

Saturday 06/03/10 Session 4 Clinical (9.00 am)

Time Allowed: 6 min Speaking

3 min Discussion

Chairpersons: Mr. Ralph Keane \& Ms. Carmel Malone

Session 4: 09.00-09.39

09.00-09.09 34. An audit of peripheral venous catheter use in surgical patients: more work to be done

S. McHugh ${ }^{1}$, S. Cowman ${ }^{2}$, S. Tierney ${ }^{1}$, A.D.K. Hill ${ }^{1}$, H. Humphreys ${ }^{3}$ (Department of Surgery, Royal College of Surgeons in Ireland $^{1}$, Department of Nursing, Royal College of Surgeons in Ireland ${ }^{2}$, Department of Microbiology, Royal College of Surgeons in Ireland ${ }^{3}$ )

09.09-09.18 35. Changing demographics in presentation for laparoscopic cholecystectomy

J. Ryan, J. Broderick, S.T. Martin, G.P. McEntee (Department of Hepatobiliary Surgery, Mater Misericordiae University Hospital, Dublin), Ireland

09.18-09.27 36. Laparoscopic resection for colorectal neoplasia-a single centre experience of 342 patients D.W. Good, J.M. O'Riordan, D. Moran, E. Guare, F.B. Keane, D.S. O'Riordain, P. Neary (Department of Colorectal Surgery, AMNCH, Tallaght, Dublin 24, Ireland)

09.27-09.36 37. Profiling breast cancer in older patients. Time to increase the population screening age?

H.D. O’Connor, M.F. Cunningham, R. Salman, M.R. Kell, T.F. Gorey, M.A. Stokes (Department of Breast Surgery, Mater Misericordiae University Hospital, Dublin 7, Ireland)

09.36-09.45 38. A decade of breast cancer screening; its impact of patient presentation to a symptomatic breast unit E. Molloy, M. Twyford, D. McCartan, Z. Al-Hilli, A.D.K. Hill (Department of Surgery, Beaumont Hospital, Dublin, Ireland)

09.45-09.54 39. A prospective evaluation of cardiovascular risk factors-are we treating to target? C. Mahon, Z. Martin, G.C. O’Brien, N. Cloete, M.P. Colgan, S.N. Haider, D.J. Moore, S.M. O'Neill, P. Madhavan (Department of Vascular and Endovascular Surgery, St James's Hospital, Dublin 8, Ireland)

09.54-10.03 40. Can augmentation index be used as an accurate tool in the diagnosis of peripheral obstructive arterial disease (POAD)? A correlation study

G.P. Duff ${ }^{1}$, O. Galvin² , J. McCormack ${ }^{2}$, P.A. Grace ${ }^{1,2}$ (Graduate Medical School, University of Limerick, Ireland $^{1}$ Department of Vascular Surgery, Mid Western Regional Hospital, Limerick, Ireland ${ }^{2}$ )

10.03-10.12 41. Re-intervention following endovascular aortic aneurysm repair: incidence in a single centre E.M. Quinn ${ }^{1}$, A.P. Coveney ${ }^{1}$, P.A. Cronin ${ }^{1}$, G. O’Brien ${ }^{1}$, G. Gosi ${ }^{1}$, D. Tuite ${ }^{2}$, G.J. Fulton ${ }^{1}$ (Departments of Vascular Surgery ${ }^{1}$ and Radiology ${ }^{2}$, Cork University Hospital, Wilton, Cork, Ireland)

10.12-10.21 42. Choosing to be a general surgeon: a quantitative study of Irish BST trainees S. McHugh ${ }^{1}$, M.A. Corrigan ${ }^{1}$, A. Sheikh ${ }^{1}$, W.A. Tanner ${ }^{2}$, A.D.K. Hill ${ }^{1}$ (Department of Surgery, Beaumont Hospital, Dublin, Ireland $^{1}$, Royal College of Surgeons, St. Stephens Green, Dublin, Ireland ${ }^{2}$ )

10.21-10.30 43. Surgent University: the establishment and evaluation of a national online clinical teaching repository for undergraduate medical students

M.A. Corrigan ${ }^{1}$, A. Sheikh ${ }^{1}$, C.J. Shields ${ }^{2}$, H.P. Redmond ${ }^{3}$, M.J. Kerin ${ }^{4}$, A.D.K. Hill ${ }^{1}$ (Department of Surgery, Beaumont University Hospital, Dublin, Ireland ${ }^{1}$, Department of Surgery, Mater Misercordiae Hospital, Dublin, Ireland ${ }^{2}$ Department of Surgery, Cork University Hospital, Cork, Ireland ${ }^{3}$ Department of Surgery, Galway University Hospital, Galway, Ireland ${ }^{4}$ )

10.30-10.39 44. Medical student education in private healthcare facilities

I. Robertson, G. Brady, A. Collins, D. O’Mara, C. Condron, A.D.K. Hill (Department of Surgery, Beaumont Hospital, Royal College of Surgeons in Ireland, Dublin 9, Ireland)

10.40-11.00

Coffee

Visit Posters, Trade Exhibition \& Poster Adjudication 


\section{Saturday 06/03/10 Session 5 Surgical Practice “Managing Change” (11.00 am)}

Time Allowed: 6 min Speaking

3 min Discussion

Chairpersons: Mr. Hugh Flood \& Mr. Kevin Barry

Session 5: 11.00-12.48

11.00-11.09 45. Changing patterns in the surgical management of parathyroid disease-a 10-year review T. McVeigh, A.J. Lowery, D. Dalton, C.A. Cowley, D. Quill, M.J. Kerin (Department of Surgery, National University of Ireland, Galway, Ireland, Clinical Science Institute, Galway University Hospital)

11.09-11.18 46. The changing face of a vascular surgical unit; 1989-2008

Z. Martin, G.C. O’Brien, N. Cloete, M.P. Colgan, S.N. Haider, D.J. Moore, S.M.O'Neill, P Madhavan (Department of Vascular and Endovascular Surgery, St James's Hospital, Dublin 8, Ireland)

11.18-11.27 47. The impact of an anaesthetic pre-operative assessment clinic on length of stay in vascular surgery patients D.B. O'Connor, M. Cotter, O. Treacy ${ }^{2}$, D. O’Malley, A. McShane ${ }^{1}$, T. Owens, S.J. Sheehan, D. Mehigan, J. Dowdall, M.C. Barry (Departments of Vascular Surgery, Anaesthesia ${ }^{1}$, Pre-operative Assessment Clinic ${ }^{2}$, St. Vincent's University Hospital, Elm Park, Dublin 4, Ireland)

11.27-11.36 48. Is laser better? A prospective outcome comparison of varicose vein therapies D.C. O’Neill, S.B. O’Neill, A.P. Coveney, D.F. O'Brien, G. Fulton (Department of Vascular Surgery, Cork University Hospital, Cork, Ireland)

11.36-11.45 49. Surgical assessment unit-an effective strategy for streamlining acute surgical admissions A. Loftus, P. Finnegan, J. Smith, P. Burke (Department of Surgery, Mid-Western Regional Hospital, Limerick, Ireland)

11.45-11.54 50. Implementation of reconfiguration of acute surgical services in the Mid-Western region J. Moloney, F.M. Smith, E.T. Condon, D. Waldron, P. Burke (Department of Surgery, Mid-Western Regional Hospital, Limerick, Ireland)

11.54-12.03 51. Rapid access colorectal clinic-5 year outcomes A. Ahmed, E. Meyers, W.O. Kirwan (Department of Academic Surgery, Cork University Hospital, Cork, Ireland)

12.03-12.12 52. The impact of caseload on practice patterns in breast cancer; evidence from the UK Breast Screening Programme N. Marshall ${ }^{1}$, R.W. Glynn ${ }^{1}$, O. Kearins ${ }^{2}$, G. Lawrence ${ }^{2}$, M.J. Kerin ${ }^{1}$ (Department of Surgery, National University of Ireland, Galway, Ireland ${ }^{1}$ West Midlands Cancer Intelligence Unit, NHS Breast Screening Programme, Birmingham ${ }^{2}$ )

12.12-12.21

53. Detrimental impact of the European working time directive (EWTD) on senior house officer operative experience K.J. Breen, A.M. Hogan, E. O’Halloran, K. Mealy (Department of Surgery, Wexford General Hospital, Wexford)

12.21-12.30 54. Preventable delays in the elective surgical patient in AMNCH, Tallaght. A retrospective study K. O’Sullivan, C. Mc Donald, P.N. Neary, E. Eguare (Department of Surgery, AMNCH, Tallaght)

12.30-12.39 55. Trends and attitudes to laparoscopic appendectomy in Ireland D.P. McCartan, F.J. Fleming ${ }^{1}$, A.D.K. Hill (Department of Surgery, Beaumont Hospital, Dublin 9, Ireland, Department of Surgery, University of Rochester Medical Centre, Rochester, New York ${ }^{1}$ )

12.39-12.48 56. Initial encounter with thoracic endovascular aneurysm repair (TEVAR) for thoraco-abdominal Pathology (TAP) in a tertiary referral vascular centre. A technical challenge of debranching, chimneys, snorkel endovascular grafts in the era of hybrid surgery?

P. Waters, G. Mann, W. Tawfick, M. Tubassam, S. Sultan (Department of Vascular Surgery, UCHG)

12.40 - Panel Adjudication for Sylvester O’Halloran Prize

13.00-13.45

Sir Thomas Myles Lecture

Presented By:

Mr. Paul Burke

CHANGE AT LIMERICK JUNCTION!

EVERYONE ON BOARD? 
Followed by Presentations of:

O'Halloran Prize (Sponsored by Leo Pharma),

Poster Prize (Sponsored by sanofi aventis)

Head \& Neck Prize (Sponsored by Sylvester O’Halloran Meeting)

Orthopaedic Prizes (Sponsored by Bayer Healthcare)

Anaesthesia Prize (Sponsored by Astellas Pharma Co.Ltd.,)

Saturday 06/03/10 Session 6 Orthopaedic Session (9.00 am)—Room D1050

Time Allowed: 7 min Speaking

3 min Discussion

Chairperson: Mr. Lester D'Souza

Session 6: $09.00-10.50$

09.00-09.10 57. The effects of resistance training and neuromuscular electrical stimulation in knee osteoarthritis-a randomized control trial

R. Bruce-Brand ${ }^{1}$, J.M. O’Byrne ${ }^{1}$, N. Moyna ${ }^{2}$ (Department of Trauma and Orthopaedics, Cappagh National Orthopaedic Hospital, Finglas, Dublin 11, Ireland ${ }^{1}$, Department of Human Health and Performance, Dublin City University ${ }^{2}$ )

09.10-09.20 58. Effect of vibration on the shear strength of impacted bone in revision hip surgery

S. Brennan ${ }^{1,2}$, K. Ryan ${ }^{1}$, D. Brabazon ${ }^{1}$, J. O'Byrne ${ }^{2}$ (Department of Orthopaedic Surgery, Cappagh Hospital, Dublin, Ireland $^{1}$, Department of Mechanical Engineering, Dublin City University, Dublin, Ireland)

09.20-09.30 59. Hip fracture in the elderly: an indicator of healthy life years

E. Keane, C. Green, C. Fitzpatrick, K. O'Rourke (Department of Trauma and Orthopaedics, St. Vincent's University Hospital, Dublin 4, Ireland)

09.30-09.40 60. Biomechanical comparison of the dynamic hip screw (DHS), the X-bolt and the DHS blade

F. O'Neill ${ }^{1}$, T. McGloughlin, F. Condon, M. Walsh (Centre for Applied Biomedical Engineering Research (CABER), Department of Mechanical and Aeronautical Engineering and Materials and Surface Science Institute (MSSI), University of Limerick, Midwestern Regional Orthopaedic Hospital Croom/Mid-Western Regional Hospital, Dooradoyle, Limerick, Ireland)

09.40-09.50 61. Hip and muscle forces in hip replacement subjects

D. O'Reilly ${ }^{1}$, O. Flannery ${ }^{2}$, M. Donoghue ${ }^{1}$, F. Condon ${ }^{2}$, M. Walsh ${ }^{1}$ (Centre for Applied Biomedical Engineering Research, Department of Mechanical and Aeronautical Engineering, University of Limerick, Limerick, Ireland ${ }^{1}$, St. Nessan's, MidWestern Regional Hospital, Croom, Limerick, Ireland ${ }^{2}$ )

09.50-10.00 62. The Irish hip fracture database: 1 year results from a Regional Hospital in Mid-West Ireland A. Daly ${ }^{1}$, T. Burke ${ }^{2}$, D. O'Farrell ${ }^{2}$, L. D’Souza ${ }^{2}$, E. Masterson ${ }^{2}$, F. Condon ${ }^{2}$ (Orthopaedic Clinical Nurse Specialist, MidWestern Regional Hospital Limerick, Ireland ${ }^{1}$ Consultant Orthopaedic Surgeons, Mid-Western Regional Hospital Limerick, Department of Orthopaedics, Mid-Western Regional Hospital, Limerick, Ireland ${ }^{2}$ )

10.00-10.10 63. Femoral neck narrowing and resurfacing arthroplasty: an early or late osteolytic phenomenon? E. Cullen ${ }^{1}$, A. Simpkin ${ }^{2}$, D. Bergin ${ }^{3}$, K. Kaar ${ }^{1}$, W. Curtin ${ }^{1}$ (Department of Orthopaedics, Merlin Park Hospital, Galway, Ireland $^{1}$, Health Research Board, Clinical Research Facility, NUI, Galway, Ireland ${ }^{2}$ Department of Radiology, Merlin Park Hospital, Galway, Ireland ${ }^{3}$ )

10.10-10.20 64. Mortality and morbidity risk factors in revision arthroplasty

N. Bandorf, D. Lui, P. Riordan, O. Jaweesh, B. Duru, D. Bennett (Department of Trauma and Orthopaedics, Mayo General Hospital, Castlebar, Department of Orthopaedics, Galway Clinic, Galway, Ireland)

10.20-10.30 65. The impaction frequency and threshold force required for femoral impaction grafting in revision hip arthroplasty-a human cadaveric biomechanical study

F. Cummins, P. Kenny, D. Kelly (Cappagh National Orthopaedic Hospital, Finglas, Dublin, Department of Bioengineering, Trinity College Dublin)

10.30-10.40 66. The effect of anticoagulants on the outcome of total knee arthroplasty I. Radovanovic, J. Queally, S. Bahari, J. Sproule, S. Henari, J. McElwain (Adelaide and Meath National Children's Hospital, Tallaght, Dublin, Ireland)

10.40-10.50 67. The use of circular external fixators for fracture stabilisation and management of fracture non-union. a review of 15 years experience

B.J. O’Neill, D.P. Moore (The Adelaide and Meath Hospital Incorporating the National Children's Hospital Tallaght, Dublin 24, Ireland)

10.50-11.10 Coffee

Visit Posters, Trade Exhibition \& Poster Adjudication 


\section{Saturday 06/03/10 Session 7 Orthopaedic Session (11.10 am) - Room D1050}

Time Allowed: 7 min Speaking

3 min Discussion

Chairperson: Mr. Dermot O'Farrell

Session 7: 11.10-13.00

11.10-11.20 68. The MRSA prevalence in trauma patients admitted from Nursing Homes; the need for glycopeptide prophylaxis C. Green, D. Hennessey, C. Fitzpatrick, L. Fenelon, K. O’Rourke (Department of Trauma and Orthopaedic Surgery, St. Vincent's University Hospital)

11.20-11.30 69. Fractures of the humeral diaphysis-axial distraction and non-union S.A. Brennan, K. Ryan, R.J. Walls, D. Murphy, P. Kenny, P. Keogh, S. O’Flannagan (Connolly Hospital, Blanchardstown, Dublin, Ireland)

11.30-11.40 70. Selective ultrasound screening for developmental displasia of hip (DDH). Effectiveness of one stop DDH clinic G.A. Naqvi, S. A. Malik, O. Adamec (Department of Orthopaedic, Our Lady of Lourdes Hospital, Drogheda, Co. Louth, Republic of Ireland)

11.40-11.50 71. Arthroscopic management of soft tissue ankle impingment S.A. Brennan, K. Ryan, F. Rahim, J. Dowling, S. Kearns (University College Hospital Galway, Ireland)

11.50-12.00 72. A risk analysis for the operative versus non-operative management of posterior malleolar fragments at 5 years E. Cullen ${ }^{1}$, A. O'Conghaile ${ }^{1}$, R. Flavin ${ }^{1}$, A. Simpkinn', D. Bergin ${ }^{3}$, D. O'Keefe ${ }^{3}$, S. Kearns ${ }^{1}$ (Department of Orthopaedics, Merlin Park Hospital, Galway, Ireland ${ }^{1}$, Health Research Board, Clinical Research Facility, NUI, Galway, Ireland ${ }^{1}$, Department of Radiology, Merlin Park Hospital, Galway, Ireland ${ }^{3}$ )

12.00-12.10 73. RhBMP-2 use in lumbar fusion surgery is associated with better leg pain scores at late follow up F.E. Rowan ${ }^{1}$, N. O'Malley ${ }^{2}$, A. Poynton ${ }^{1}{ }_{3}^{2}$ (Department of Orthopaedics and Spinal Surgery, Mater Private Hospital, Dublin 7, Ireland, Ireland ${ }^{1}$, Department of Orthopaedics and Spinal Surgery, Cappagh National Orthopaedic Hospital, Dublin 11, Ireland ${ }^{2}$ )

12.10-12.20 74. Anterior cervical disc replacement: early clinical results

S. Munigangaiah, K. C. Cronin, J.P. McCabe (Department Of Trauma and Orthopaedic Surgery, University College Hospital Galway, Newcastle, Galway, Ireland)

12.20-12.30 75. A novel technique for determining transverse skin incision site for anterior cervical spine surgery

C. Kennedy, M. Leonard, H. Heneghan, J.P. McCabe (Department of Trauma and Orthopaedic Surgery, University Hospital Galway, Ireland)

12.30-12.40 76. Outcomes of traction splinting of closed proximal phalangeal fractures

S.M. O’Meara, D.T. Cawley, F.J. Shannon (Dept of Orthopaedic and Trauma Surgery, Galway University Hospital, Galway, Ireland)

12.40-12.50 77. Free hand versus novel specialised jig guidance for the passing of intra-medullary wires in olecranon fracture fixation: a comparative study

A. Gheiti, D.C. Molony, J. Kennedy, J.H. Mullett (Cappagh National Orthopaedic Hospital, Finglas, Dublin 11, Republic of Ireland)

12.50-13.00 78. Radiological outcomes of distal radius extra-articular fragility fractures treated with extra-focal kirschner wires C. Kennedy ${ }^{1}$, M.T. Kennedy ${ }^{2}$, D. Niall ${ }^{2}$, A. Devitt ${ }^{1}$ (Department of Orthopaedic Surgery, University College Hospital, Galway, Republic of Ireland ${ }^{1}$, Department of Orthopaedic Surgery, Midland Regional Hospital, Tullamore, Co Offaly, Republic of Ireland ${ }^{2}$ )

12.40 - Panel Adjudication for Sylvester O'Halloran Prize

13.00-13.45

Sir Thomas Myles Lecture

Presented By:

Mr. Paul Burke

CHANGE AT LIMERICK JUNCTION!

EVERYONE ON BOARD? 
Followed by Presentations of:

O'Halloran Prize (Sponsored by Leo Pharma)

Poster Prize (Sponsored by sanofi aventis)

Head \& Neck Prize (Sponsored by Sylvester O'Halloran Meeting)

Orthopaedic Prizes (Sponsored by Bayer healthcare)

Anaesthesia Prize (Sponsored by Astellas Pharma Co. Ltd.,)

Saturday 06/03/10 Session 8 Anaesthesia Session (10.30 am)_Room C1059

Time Allowed: 7 min Speaking

3 min Discussion

Chairperson: Professor Dominic Harmon

Session 8: 10.30-11.40

10.30-10.40 79. Comparison of analgesic requirement in first $24 \mathrm{~h}$ following total knee arthroplasty in patients with or without Periartuclar Chirocaine administration; retrospective analysis of case notes

A. Memon, S. Umar, B. Suleman, R. Gul, J. Harty, M. Dolan (St Mary's Orthopaedic Hospital, Cork University Hospital, Cork, Ireland)

10.40-10.50 80. Influence of barometric pressure on pain severity in end stage osteoarthritis

S.A. Brennan ${ }^{1}$, K. Ryan ${ }^{1}$, T. Harney ${ }^{1}$, C. Gormley ${ }^{2}$, N.A. Rahim ${ }^{1}$, F. Shannon ${ }^{1}$ (University College Hospital Galway, Ireland $^{1}$, School of Mathematical Science, University College Dublin, Ireland ${ }^{2}$ )

10.50-11.00 81. Iliolumbar syndrome: sonographic anatomy and injection technique

V. Alexiev, D. Harmon (Department of Anaesthesia and Pain Medicine, Mid-Western Regional Hospital, Dooradoyle, Limerick, Ireland)

11.00-11.10 82. Audit on pre-preparation of "emergency drugs": financial impact, awareness of costs and practice of pre-preparation J. Steiner ${ }^{1}$, C. Maharaj ${ }^{2}$, W. O’Brien ${ }^{1}$ (Mid-Western Regional Hospital, Limerick, Ireland ${ }^{1}$, University Hospital, Galway, Ireland $^{2}$ )

11.10-11.20 83. Safer surgery: how accurate are we at predicting intraoperative blood loss?

C. Egan, J.G. Solon, D.A. McNamara (Dept of Surgery, Beaumont Hospital, Beaumont, Dublin 9, Ireland)

11.20-11.30 84. Assessment of balance with shoulder stabilization sling using computerized dynamic posturography A. Memon*1 ${ }^{1}$, D. Lui ${ }^{2}$, H. Mullett ${ }^{2}$, S. Kwan ${ }^{3}$ (Cork University Hospital ${ }^{1}$, Beaumont Hospital Dublin, Ireland ${ }^{2}$, Royal College of Surgeons Ireland ${ }^{3}$ )

11.30-11.40 85. Use of pumpless extracorporeal interventional lung assist devices (PEILADs) in patients with H1N1 type A influenza P. Johnson, A. Westbrook (Intensive Care Unit, St James's Hospital, Dublin 8, Ireland) 
Poster Session-Sponsored by sanofi aventis.

1. Digit ratio (2D:4D) as a predictor of breast cancer predisposition

A. Devine, J. Kelly, H. Heneghan, R. Dwyer, M. Kerin (Department of Surgery, National University of Ireland, Galway, Ireland)

2. Abdominal aortic aneurysms mechanical property quantification using acoustic radiation force impulse

A. Tierney ${ }^{1}$, D. Dumont ${ }^{1}$, A. Callanan ${ }^{2}$, T. M. McGloughlin ${ }^{2}$ (Duke University, North Carolina, USA ${ }^{1}$, Centre for Applied Biomedical Engineering Research (CABER), Department of Mechanical and Aeronautical Engineering, Materials and Surface Science Institute (MSSI), University of Limerick, Ireland ${ }^{2}$ )

3. Serum antibiotic prophylaxis for transrectal ultrasound guided biopsy of prostate-a prospective comparative study of two regimes involving $>1000$ men

G. Nason, R.P. Manecksha, I.M. Cullen, E. McEvoy, T.E.D. McDermott, R. Flynn, R. Grainger, J.A. Thornhill (Department of Urology, Adelaide and Meath incorporating the National Children's Hospital, Dublin 24, Ireland)

4. Site distribution of melanoma. A retrospective study examining the relationship between site of cutaneous melanoma and occupational exposure to sunlight

T. Burke, S. Potter, D. Joyce, J. McCann, J. Kelly, A. Hussey, P. Regan, M. Kerin (Department of Plastic, Reconstructive and Aesthetic Surgery, Department of General Surgery, University Hospital, Galway, Ireland)

5. Effect of previous percutaneous coronary intervention on cardiac surgery-a single-centre study

L. Sutton ${ }^{1}$, S. Early ${ }^{1}$, M. Tolan ${ }^{1}$, V. Young ${ }^{1}$, J. Moriarty ${ }^{2}$, E. McGovern ${ }^{1}$ (Departments of Cardiac Surgery ${ }^{1}$ and Anaesthesia ${ }^{2}$, St James's University Hospital)

6. The Prevalence of PSA 'Self-Testing' among consultant urologists

N.F. Davis, B.B. McGuire, H.D. Flood (Department of Urology, Mid-Western Regional Hospital, Limerick, Ireland)

7. Day-case laporoscopic surgery: how can we improve?

E. Ibrahim, M. Salama, A. Lockley, M. Nolan, N. Kinsella, I. Ahmed (Our Lady's Hospital, Navan, Co. Meath)

8. Surgical services, a victim of criminal minds

J.G. Hogan, M. McCafferty, P. Keeling (James Connolly Memorial Hospital, Blanchardstown, Dublin 15, Ireland)

9. Comparing clinician and HIPE based discharge summary coding and its impact on funding within a University Teaching Hospital Surgical Department

R. Murphy, T. McVeigh, P. Waters, G.T. O’Donoghue, M. Kerin (Department of Endocrine and Breast Surgery, University College Hospital Galway, Newcastle Road, Galway, Ireland)

10. Assessment of suitability of rectal cancer for a laparoscopic approach-a single centre experience of 122 patients

D.W. Good, J.M. O'Riordan, D. Moran, F.B.V. Keane, E. Eguare, D.S. O'Riordain, P. Neary (Department of Colorectal surgery, AMNCH, Tallaght, Dublin 24, Ireland)

11. Chylothorax and congenital cardiac surgery; a single centre study of 452 paediatric patients

S. Halpenny, S. Early, C. McMahon, A.E. Wood, M.J. Redmond, D. Mannion, L. Nolke (Departments of Surgery and Anaesthesia, Our Lady's Hospital for Sick Children, Crumlin, Dublin, Ireland)

12. Investigation of potential role of CCL5 in breast cancer progression

M.C. Hartmann, R.M. Dwyer, M. Costello, M.J. Kerin (Department of Surgery, National University of Ireland, Galway, Ireland)

13. Patterns of disease recurrence in an Irish breast cancer cohort

S. Lewis, D.P. McCartan, Z.M. AlHilli, A.D.K. Hill (Department of Surgery, Beaumont Hospital, Dublin 9, Ireland)

14. Colonic interposition: the Beaumont experience

J.C. Bolger, E. Myers, P. Broe (Department of Surgery, Beaumont Hospital, Beaumont, Dublin 9, Ireland)

15. Review of APR in Beaumont Hospital 1996-2006: benign disease represents an important component of operative experience D.R. Gough, J. Keogh, A. Hanly, N. McCawley, J. Deasy, D.A. McNamara (Department of Colorectal Surgery, Beaumont Hospital, Dublin, Ireland)

16. A "Natural Killer" role for the omentum in tumour immunity

D.B. O'Connor ${ }^{1}$, D.O' Shea ${ }^{2}$, C. O'Farrelly ${ }^{3}$, L. Lynch $^{2,4}$, D.C. Winter ${ }^{1}$ (Surgery ${ }^{1}$, St. Vincent's University Hospital, Elm Park Dublin 4, Ireland, Obesity and Immunology ${ }^{2}$, St. Vincent's University Hospital, Dublin 4, Ireland, Trinity College ${ }^{3}$, Dublin 2, Ireland, Harvard Medical School ${ }^{4}$, Boston, USA)

17. Why routine ICU admission after elective open infrarenal abdominal aortic aneurysm (AAA) repair is no longer evidence based practice

D. Ryan, G. McGreal (Department of Vascular Surgery, Mercy University Hospital, Grenville Place, Cork, Ireland)

18. Surgical procedures and the internet. The challenge of ensuring patients are accurately informed

E.K. Walsh, F. Hand, J.O. Murphy, M.R. Kell (Eccles Breast Screening Unit, Mater Misericordiae University Hospital, BreastCheck, Eccles Street, Dublin 7, Ireland)

19. Radiation safety awareness... or not?

M. Murphy, Z. Martin, G.C. O’Brien, N. Cloete, M.P. Colgan, S.N. Haider, P. Madhavan, S.M. O’Neill, D.J. Moore (Department of Vascular and Endovascular Surgery, St James's Hospital, Dublin 8, Ireland)

20. The use of sequential compression biomechanical device in critical limb ischemia patients with un-re-constructable peripheral vascular disease. A 5 years experience in a tertiary referral vascular centre

N. Hamada, S. Sultan (University College Hospital Galway (UCHG), Western Vascular Institute (WVI), Newcastle, Galway, Ireland) 


\section{SESSION 1: SCIENTIFIC}

\section{FAECAL INCONTINENCE: WHERE IS THE SENSE IN IT ALL?}

\author{
C. Peirce ${ }^{1}$, C. O’Herlihy ${ }^{1,2}$, P.R. O’Connell ${ }^{1,3}$, J.F.X. Jones ${ }^{1}$ \\ School of Medicine and Medical Science, University College Dublin, \\ Dublin 4, Ireland ${ }^{l}$, National Maternity Hospital, Holles Street, \\ Dublin, Ireland ${ }^{2}$, Professorial Surgical Unit, St. Vincent's University \\ Hospital, Dublin 4, Ireland ${ }^{3}$
}

Sacral nerve stimulation is a therapy which is changing the treatment algorithm for faecal incontinence. The mechanism of action is poorly understood. Recently, it has been hypothesised that neuromodulation occurs at the level of the sensory neuron. Experimental models of neuropathic faecal incontinence have been established [1,2]. The aim of this study was to determine the effect of direct crush and compression injury on inferior rectal nerve (IRN) sensory neurons using a nuclear marker of neuronal injury, activating transcription factor 3 (ATF-3). Eighteen female virgin Wistar rats were assigned equally to thee groups: an unoperated control group, a direct IRN crush group (acting as a positive control) and an IRN balloon compression group. On postoperative day 5, dorsal root ganglia (DRG) L6-S2 and the corresponding lumbosacral spinal cord were harvested. DRG were cryosectioned (20 $\mu \mathrm{m}$ thickness), stained immunocytochemically for ATF-3 and the extent of nuclear labelling analysed. The direct IRN crush and IRN balloon compression groups had greater ATF-3 nuclear labelling than the unoperated control group $(p=0.005$, Kruskal-Wallis test). Estimated total DRG ATF-3 nuclear labelling was also significantly higher in the direct IRN crush $[248(86,1,194)$; median (25th, 75th centiles)] and IRN balloon compression groups [88 $(30,408)]$ compared with the unoperated control group $[14(4,36)]$. ATF-3 nuclear staining of IRN sensory neurons is specific to injury in experimental models of faecal incontinence. This finding suggests that these neurons are the most vulnerable to injury and thus likely play the key role in the neuromodulatory effects of sacral nerve stimulation.

(1) Healy CF, O'Herlihy C, O'Brien C, O'Connell PR, Jones JF (2008) Experimental models of neuropathic fecal incontinence: an animal model of childbirth injury to the pudendal nerve and external anal sphincter. Dis Colon Rectum 51: 1619-16126.

(2) Peirce C, Healy CF, O'Herlihy C, O'Connell PR, Jones JF (2009) Reduced somatosensory cortical activation in experimental models of neuropathic fecal incontinence. Dis Colon Rectum 52:1417-1422.

Conflict of interest: None

Disclosures: None

\section{A SYSTEMIC MIRNA SIGNATURE PREDICTIVE OF EARLY BREAST CANCER}

\author{
H.M. Heneghan, N. Miller, R. Kelly, M. Nugent, M.J. Kerin \\ Department of Surgery, National University of Ireland, Galway, \\ Ireland
}

The potential of miRNAs as novel tumour markers has been the focus of much recent attention due to their tissue specificity and unique ability to predict clinicopathological parameters with superior accuracy to mRNA profiling. It is unknown whether the systemic miRNAs recently reported to be altered in cancer are tumour specific or a general cancer phenomenon. We wished to determine if circulating 'oncomirs' were cancer specific and to identify systemic miRNA signatures predictive of specific cancers.

Using a novel isolation technique and RQ-PCR, expression levels of nine oncogenic miRNAs were quantified in blood specimens from 163 cancer patients (breast, colon, renal, prostate, melanoma) and 44 age-matched disease free controls. Advanced QBase software and SPSS were used for biostatistical analysis of data.

Expression of general oncomirs, let 7a and miR-21, were similarly expressed in all cancers. Other specific miRNAs were remarkably different between cancer types. MiR-10b and miR-145 were significantly decreased in circulation of colon cancer patients $(p=0.000$ and $p=0.017$, respectively). MiR-195, miR-342 and miR-181c were breast cancer specific; elevated levels of these three miRNAs could reliably differentiate breast cancers from controls, and other malignancies, with high sensitivities (AUC by ROC analysis: $0.941,0.963$, and 0.926 , respectively). A 3-miR signature was identified which was predictive of intrinsic subtype.

These findings suggest that individual malignancies display specific circulating miRNA profiles, which could aid in early diagnosis and discriminating between cancers. This is of major clinical importance as it illustrates the potential for systemic miRNAs as sensitive, specific and noninvasive cancer biomarkers.

Conflict of interests: None

Disclosures: None

\section{BENEFIT OF ADJUVANT CHEMOTHERAPY IN LUMINAL A BREAST CANCERS}

\author{
A. McDermott ${ }^{1}$, H.M. Heneghan ${ }^{1}$, C. Malone ${ }^{1}$, R. McLaughlin ${ }^{1}$, \\ K.J. Sweeney ${ }^{2}$, M.J. Kerin ${ }^{1}$
}

Department of Surgery, Galway University Hospital ${ }^{I}$ National Breast Cancer Screening Programme, University College Hospital, Galway, Ireland ${ }^{2}$

Sorlie's molecular portrait of breast tumours explained the phenotypic heterogeneity of breast cancers by demonstrating diversity in gene expression. The commonest breast cancer subtype, Luminal A (ER/PR+, HER2-), generally carries a good prognosis however within this subgroup there is marked diversity in outcome. Debate continues as to their need for adjuvant chemotherapy. We aimed to assess outcomes of Luminal A cancers in order to determine prognostic factors which may guide future treatment strategies for this subgroup.

1,355 breast cancer patients with known molecular subtype were identified; 965 were Luminal A, of whom 57\% received adjuvant chemotherapy. Clinicopathological details were reviewed, and current outcome determined. Kaplan-Meir and multivariate analyses were performed to identify tumour/patient characteristics indicative of poor response to adjuvant chemotherapy.

Median follow-up was 48 months. Overall and disease free survival were significantly better for those who received adjuvant chemotherapy (OS: 48 vs. 42 months, $p=0.001$. DFS: 45 vs. 37 months, $p<0.001)$. Patients whose disease progressed despite chemotherapy had poorer prognostic indicators at time of diagnosis, compared to their Luminal A counterparts who remained disease free: significantly greater tumour size $(30.34 \mathrm{~mm} \mathrm{vs.} 25.0 \mathrm{~mm}$, $p=0.01), 69 \%$ node positive and $59.4 \%$ had stage 3 or 4 disease (chi square $<0.001$ ). Of the subgroup that remained disease free, $40 \%$ had not received adjuvant chemotherapy. 
Adjuvant chemotherapy is beneficial in the cohort of Luminal A's with advanced disease at diagnosis. However it is critically importance to identify this particular subgroup and spare those with early disease the toxicity of treatment form which they were unlikely to derive further benefit.

Conflict of interests: None

Disclosures: None

\section{HYPOXIA INHIBITS COLONIC ION TRANSPORT THROUGH ACTIVATION OF AMP KINASE.}

\author{
D. Collins ${ }^{1,2,3}$, S. Kopic ${ }^{3}$, J. Bachlechner ${ }^{3}$, A.M. Hogan ${ }^{1,2}$, \\ M. Medani ${ }^{1,2}$, R. Kennelly ${ }^{1,2}$, J.P. Geibel ${ }^{3}$, D.C. Winter ${ }^{1}$ \\ Institute for Clinical Outcomes Research and Education (iCORE), \\ St Vincent's University Hospital, Dublin, Ireland ${ }^{l}$, College of Life \\ Sciences \& Conway Institute, UCD, Dublin, Ireland ${ }^{2}$, \\ Department of Surgery, Yale University, New Haven, CT, USA ${ }^{3}$
}

Background: Colonic hypoxia is the endpoint in the pathogenesis of many surgical disorders including acute ischemia, colonic obstruction and inflammatory bowel disease. Acute hypoxia is known to modulate colonic epithelial ion transport however the underlying mechanisms are poorly understood. Adenosine monophosphate (AMP)-activated protein kinase (AMPK) is a metabolic energy sensor, present in enterocytes, that regulates cell metabolism under conditions of stress. We hypothesised that activation of AMPK is an early event in the response to hypoxia, mediating of the acute effects of hypoxia in this setting.

Methods: Human mucosal epithelium from colectomy specimens was mounted in Ussing chambers. Chloride secretion was quantified as changes in short circuit current $(\Delta \mathrm{Isc})$ in $\mu \mathrm{A} \mathrm{cm} \mathrm{cm}^{2}$. Hypoxia was induced by switching to nitrogen perfusion $\left(95 \% \mathrm{~N} 2 / 5 \% \mathrm{CO}_{2}\right)$. Rat colonic crypts were isolated by calcium chelation, and intracellular intracellular chloride $(\mathrm{Cl}) \mathrm{i}$ was measured by MQAE fluorescence. The contribution of AMPK in this hypoxic response was evaluated by pretreating tissues with the AMPK inhibitor Compound C. Institutional review board approval was granted for this study.

Results: Hypoxia decreased chloride secretion in human colon and isolated crypts $(n=8, P<0.03)$. Pre-treatment of tissues with compound $\mathrm{C}$ significantly reversed these effects $(n=8, \mathrm{P}<0.04)$.

Conclusion: This study demonstrates that acute hypoxia inhibits electrogenic chloride secretion in rat and human colon through an AMPK mediated mechanism. AMPK is therefore a key component of the adaptive response to mucosal hypoxia and may represent a potential therapeutic target in ischemic states.

Conflict of interest: None

Disclosures: The study was supported by a research grant from IRCSET.

\section{JUNCTIONAL ADHESION MOLECULE-A (JAM-A): A POTENTIAL TARGET FOR BREAST CANCER ANTI-PROLIFERATIVE THERAPY?}

\section{G.O. Offiah, E.A. McSherry, A.D.K. Hill, A.M. Hopkins \\ Department of Surgery, Royal College of Surgeons in Ireland, Dublin 9, Ireland}

Background: JAM-A regulates numerous cellular processes in epithelial tissues including the breast. Our laboratory recently reported a significant correlation between increased JAM-A expression and poor survival of breast cancer patients (McSherry et al. 2009). Thus, the aim of this study was to elucidate the role of JAM-A in regulating cell polarity, morphologic transformation and proliferation during breast cancer progression.
Methods: The HMT-3522 series of S1 (normal) and T42 (invasive) cell lines was utilised in this study. To assess effects on proliferation, MTT assays were conducted on S1 and T42 cells after either JAM-A inhibitory antibody or negative control treatments. To determine effects on acinar polarization, 3-dimensional (3D) cultures of S1 and T42 cells, treated with JAM-A inhibitory antibody or negative control, were immunostained for F-actin and JAM-A and examined by confocal microscopy.

Results: At days 9 and 11, the proliferation of JAM-A inhibitory antibody-treated S1 and T42 cells was reduced compared to control treated cells $(P<0.05)$. In 3D culture, $\mathrm{S} 1$ cells formed hollow spheroids, resembling breast acini in vivo; while T42 cells formed unpolarized structures. S1 and T42 cells treated with JAM-A inhibitory antibody showed reductions in spheroid diameter relative to control-treated cells $(P<0.05)$. Interestingly, invasive T4-2 cells treated with JAM-A inhibitory antibody exhibited a partial normalisation toward an S1 phenotype.

Conclusions: Our results demonstrate that JAM-A inhibition decreases proliferation and partially restores polarity in breast cancer cells. Therefore, we speculate that JAM-A, may be a future target in breast cancer treatment.

Conflict of interest: None

Disclosures: None

\section{BACTERIAL SUPERANTIGENS ENHANCE THE IN VITRO PRO-INFLAMMATORY CYTOKINE RELEASE AND IN VIVO LETHALITY OF THE TLR2 AGONIST BACTERIAL LIPOPROTEIN}

\author{
D.E. Kearney, J.H. Wang, H.P. Redmond \\ Department of Academic Surgery, Cork University Hospital, Cork, \\ Ireland
}

Bacterial superantigens are gram-positive exotoxins that induce proinflammatory cytokine release in vitro, cause lethal shock in vivo, and can be detected in the bloodstream of critically ill patients. They are also known to have a powerful synergy with the gram-negative TLR4 agonist, endotoxin. The aim of this research was to investigate the synergistic relationship between superantigens and the gram-positive TLR2 agonist, bacterial lipoprotein (BLP).

Human monocytes and peripheral blood mononuclear cells (PBMCs) were isolated from healthy volunteers. Priming of human monocytes or PBMCs with superantigens significantly enhanced the release of pro-inflammatory cytokines TNF- $\alpha$ and IL- 6 in response to either BLP or endotoxin $(p<0.001)$. Superantigens significantly upregulated the expression of TLR2 and TLR4 on monocytes as determined by FACScan analysis. The priming effect of superantigens could be blocked using inhibitors to $\mathrm{p} 38$ during the priming phase as opposed to NF- $\kappa \mathrm{B}$ or ERK inhibition. This was consistent with higher expression of phosphorylated p38 after superantigen priming and BLP or endotoxin stimulation. C57BL/6 mice with superantigen priming $(10 \mu \mathrm{g} /$ mouse) had significantly higher mortality $(10 / 10)$ when challenged with BLP $(600 \mu \mathrm{g} / \mathrm{mouse})$ compared to mice without superantigen priming $(0 / 10)$. Mice given superantigen alone did not demonstrate any signs of illness. Mice challenged with superantigen and BLP had significantly higher levels of TNF- $\alpha$ at $90 \mathrm{~min}$ and $4 \mathrm{~h}$ compared to mice challenged with superantigen or BLP alone.

Bacterial superantigens enhance the in vitro pro-inflammatory cytokine release and the in vivo lethality of BLP. This novel synergy may help to explain the massive pro-inflammatory cytokine release seen in superantigen mediated septic shock.

Conflict of interest: None

Disclosures: None 
7 UNRAVELLING A NOVEL ROLE OF MYELOIDRELATED PROTEINS 8/14 (MRP 8/14) IN SELFTOLERANCE AND CROSS-TOLERANCE TO GRAM-NEGATIVE AND GRAM-POSITIVE BACTERIA

\author{
A.P. Coveney, J.H. Wang, H.P. Redmond
}

Department of Academic Surgery, Cork University Hospital, Wilton, Cork, Ireland

This study examines the ability of myeloid-related protein 8 (Mrp8), the active component of Mrp8/14 complex, to induce self-tolerance and cross-tolerance to BLP and LPS.

Murine peritoneal macrophages and bone marrow derived macrophages (BMMs) from $\mathrm{C} 3 \mathrm{H} / \mathrm{HeN}$ mice were isolated and stimulated with increasing doses of murine Mrp8 for $18 \mathrm{~h}$, washed and restimulated with $5 \mu \mathrm{g} / \mathrm{ml}$ of Mrp8 for $6 \mathrm{~h}$. TNF- $\alpha$ levels of supernatants from naïve macrophages and pre-stimulated macrophages were compared. Mrp8 tolerised macrophages were also re-stimulated with BLP and LPS to investigate if cross-tolerance occurs. The role of Toll-like receptors TLR2 and TLR4, was examined by repeating the experiment with TLR2-/- and TLR4-/- mice. The experiment was repeated also using human monocytes.

Mrp8 stimulation triggers TNF- $\alpha$ release. Prestimulation with Mrp8 attenuates this TNF- $\alpha$ release, in both murine macrophages and human monocytes. TNF- $\alpha$ release decreased by $88 \%$ in BMMs pre-stimulated with $5 \mu \mathrm{g} / \mathrm{ml}$ of Mrp8 compared to naive macrophages when both were re-stimulated with the same Mrp8 dose $(p<0.0005)$. The tolerance effect is both dose and time dependent. TLR4 is essential but TLR2 also contributes to the tolerising effects of Mrp8. Pre-stimulation with $1 \mu \mathrm{g} / \mathrm{ml}$ of Mrp8 leads to a $26 \%$ reduction in TNF- $\alpha$ release when cells are stimulated with $100 \mathrm{ng} / \mathrm{ml}$ of BLP $(p<0.002)$ confirming cross tolerance with BLP. Human monocytes were more sensitive to the tolerising effects.

Mrp8 induces self-tolerance and cross-tolerance to BLP, and LPS in a dose and time dependent manner in murine macrophages and human monocytes identifying a potential mechanism for attenuating the inflammatory response

Conflict of interest: None

Disclosures: None

\section{URINARY BLADDER MATRIX TUBULAR SCAFFOLDS AS A TISSUE ENGINEERED VASCULAR GRAFT}

\author{
A. Callanan, M.T. Walsh, T.M. McGloughlin
}

Centre for Applied Biomedical Engineering Research (CABER), Department of Mechanical and Aeronautical Engineering, Materials and Surface Science Institute (MSSI), University of Limerick, Ireland

Introduction: Arterial diseases are a common cause of death in the western world. A common treatment procedure used is bypass grafting, which currently utilize synthetic graft materials, internal thoracic artery, and autologous vein. An alternative to these treatment approaches are tissue engineered materials. Urinary bladder matrix (UBM) is one such as material which is classed as a naturally derived extracellular matrix (ECM). The main aim of this research is to examine the suitability of UBM as a vascular graft by the study of matrix metalloproteinase 2 (MMP2), tissue inhibitor of metalloproteinase 2 (TIMP2) gene expression and cellular growth of human
Aortic endothelial cells (HAEC's) when attached to Urinary Bladder Matrix (UBM) under static and aortic steady flow conditions.

Materials and methods: The UBM tubes were seeded with human aortic endothelial cells (HAEC) at a density of $0.4 \times 106 / \mathrm{cm}^{2}$ using a novel roller bottle system. The seeded tubes were placed into a bioreactor flow chamber under steady shear flow for $24 \mathrm{~h}$. MMP and TIMP2 gene expression, indicators of angiogenesis and arterial remodelling were measured using real time RT-PCR.

Results and conclusion: The UBM tubes showed high levels of cell attachment and proliferation on the surface, which was determined using live/dead assay after the flow tests. Gene expression of TIMP2 was down-regulated after the application of the steady flow. MMP2 Gene expression was up regulated in both the steady and flow experiments. With an angiogenic gene being up-regulated, it indicates the onset of remodelling in the UBM material, which may be an indication of arterial formation.

Conflict of interest: None

Disclosures: None

\section{ARE URINARY BLADDER VOLUMES MAINTAINED USING XENOGENIC URINARY BLADDER MATRIX (UBM) GRAFTS IN OVINE PARTIAL CYSTECTOMY MODELS?}

\author{
N.F. Davis ${ }^{1,2}$ A. Callanan ${ }^{2}$, B.B. McGuire ${ }^{1}$, H.D. Flood ${ }^{1}$ \\ T.M. McGloughlin ${ }^{2}$
}

Department of Urology, Mid-Western Regional HospitaL, Limerick, Ireland ${ }^{l}$, The Centre for Applied Biomedical Engineering Research, University of Limerick, Castletroy, Co. Limerick, Ireland ${ }^{2}$

Introduction: The urinary bladder's high viscoelasticity and contractile properties mean that it is a difficult organ to augment or replace. The bladder is highly compliant and can accommodate large intravesical volume changes while maintaining almost constant pressure values. Functional neo-bladders have been created with xenogenic biomaterials, however, decreased post-operative bladder capacity secondary to the xenograft's poor distensibility is a persistent clinical limitation. This study aims to address volume limitations by applying larger xenografts to smaller bladder defects.

Methods: Mean bladder volumes at 5, 10, 15 and $20 \mathrm{mmHg}$ were measured after the genitourinary tract of female lambs was dissected ( $n=5$, age $7 / 12$ ). Partial cystectomy was then performed (average size $9 \mathrm{~cm}^{2}$ ) and the cystectomy defect was repaired twice. The defect was initially repaired with a $9 \mathrm{~cm}^{2}$ segment of xenogenic urinary bladder matrix (UBM) and subsequently with a $36 \mathrm{~cm}^{2}$ segment of UBM. Bladder volume measurements were repeated at 5, 10, 15 and $20 \mathrm{mmHg}$ and compared to baseline values.

Results: Bladder capacity decreased by a mean of $39.6 \%(105.8 \mathrm{ml})$ $(n=5$, range: $37.5-41.3 \%)$ throughout all pressure changes after the $9 \mathrm{~cm}^{2}$ UBM replaced an equal sized bladder wall defect. In comparison, bladder volumes improved when the $9 \mathrm{~cm}^{2}$ bladder wall defect was replaced with a $36 \mathrm{~cm}^{2}$ segment of UBM but remained consistently lower than baseline values by a mean of $18.6 \%$ $(51.75 \mathrm{ml}) \quad(n=5$, range: $16.1-19.9 \%)$ throughout all pressure changes.

Conclusion: This study demonstrates that a larger graft surface-area can improve bladder volume; however, baseline volumes were not achieved despite a fourfold increase in the graft's surface area after partial cystectomy.

Conflict of interest: None

Disclosures: None 


\section{STRENGTH OF ATHEROSCLEROTIC PLAQUE IN CAROTID ARTERY}

\author{
M. Lawlor ${ }^{1}$, E. Kavanagh ${ }^{2}$, P.A. Grace ${ }^{2}$, M. Walsh ${ }^{1}$
}

Centre for Applied Biomedical Engineering Research, University of Limerick, Ireland ${ }^{l}$, Department of Vascular Surgery, Mid-Western Regional Hospital, Limerick, Ireland ${ }^{2}$

Atherosclerotic disease is a leading cause of death and disability. Carotid plaque rupture is responsible for the majority of ischematic strokes in the developed world. The treatment of carotid atherothrombosis is evolving and may require medical intervention by either surgical carotid endarterectomy or percutaneous carotid stenting. The biomechanical properties of the plaque may contribute to the risk of embolisation during carotid artery angioplasty and stenting.

The strain of the individual carotid plaques, freshly harvested postendarterectomy, was tested in response to an applied circumferential force by using a uniaxial tensile tester. The true strain value recorded per sample indicates the strain accommodated by the plaque prior to rupture.

The strain results show a marked heterogeneity in plaque strain capability to an applied circumferential force. The true strain does predict susceptibility to emboli during carotid stenting. $89 \%$ of the population (17 of 19 patients) analysed would suffer plaque rupture when attempting to achieve an $80 \%$ lumen during the stenting surgical procedure. Ultrasound grey-scale median characteristics of plaque did not correlate with plaque strain or rupture threshold values.

Strain characteristics of carotid plaque suggest that plaque rupture may occur in a large proportion of patients at forces generated by current angioplasty and stenting techniques.

(1) Rosamund et al. (2007) "Heart disease and stroke statistics-2007 update: a report from the American Heart Association Statistics Committee and Stroke Statistics Subcommittee. Circulation 115:169-171.

(2) Kolodgie et al. (2007) Angioplasty and stenting for atherosclerotic cerebrovascular disease, 17:285-301.

Conflict of Interest: None

Disclosures: None

Table 1 Plaque-type strength per patient tested $(n=19)$

\begin{tabular}{|c|c|c|c|c|c|c|}
\hline $\begin{array}{l}\text { Disease } \\
\text { type }\end{array}$ & $\begin{array}{l}\text { Plaque } \\
\text { label }\end{array}$ & $\begin{array}{l}\% \\
\text { Frequency } \\
\text { of } \\
\text { occurrence }\end{array}$ & $\begin{array}{l}\% \\
\text { Strain to } \\
\text { fracture } \\
\text { (Min) }\end{array}$ & $\begin{array}{l}\% \\
\text { Strain to } \\
\text { fracture } \\
\text { (Max) }\end{array}$ & $\begin{array}{l}\text { Stress to } \\
\text { fracture } \\
\text { (Min) } \\
(\mathrm{MPa})\end{array}$ & $\begin{array}{l}\text { Stress to } \\
\text { fracture } \\
(\mathrm{Max}) \\
(\mathrm{MPa})\end{array}$ \\
\hline 1 & Soft & 0 & - & - & - & - \\
\hline $1 \& 2$ & Soft & 0 & - & - & - & - \\
\hline 2 & Mixed & 16 & 52.87 & 65.87 & 5.26 & 40.25 \\
\hline $2 \& 3$ & Mixed & 26 & 19.76 & 86.90 & 10.53 & 41.37 \\
\hline 3 & Mixed & 11 & 33.38 & 58.55 & 2.45 & 23.54 \\
\hline $3 \& 4$ & Hard & 42 & 34.31 & 119.06 & 1.70 & 49.18 \\
\hline 4 & Hard & 5 & 51.27 & 51.27 & 27.55 & 27.55 \\
\hline
\end{tabular}

\section{OVEREXPRESSION OF IRAK-1 PREVENTS BLP-INDUCED TOLERANCE BYPROMOTING NF-KB P65 NUCLEAR TRANSACTIVATION}

\author{
J.H. Liu, C.H. Li, H.P. Redmond, J.H. Wang \\ Department of Academic Surgery, Cork University Hospital, Cork, \\ Ireland
}

Tolerance to bacterial cell-wall components including gram-positive bacterial lipoprotein (BLP) represents an essential regulatory mechanism during bacterial infection. BLP activates monocytes/ macrophages through TLR2-mediated signal pathway. We demonstrated that BLP-induced tolerance, characterised by down-regulated proinflammatory cytokine expression, is associated with reduced IRAK-1(interleukin-1 receptor-associated kinase 1) protein expression. However, it remains unclear whether IRAK-1 is a key molecule responsible for BLP-induced tolerance.

Human monocytic THP-1 cells were preincubated with culture medium (naive) or $100 \mathrm{ng} / \mathrm{ml}$ BLP (BLP-tolerised) for $24 \mathrm{~h}$, and further stimulated with $1,000 \mathrm{ng} / \mathrm{ml}$ BLP for various time points. For IRAK-1 overexpression, BLP-tolerised cells were transfected with plasmid encoding IRAK-1 or empty vector as the control. Chromatin immunoprecipitation (ChIP) was performed to assess nuclear transactivation of NF-kB p65 at the TNF-a and IL-6 promoters, and ELISA was used to measure proinflammatory cytokine production.

BLP stimulation caused a rapid recruitment of NF-kB p65 to both TNF-a and IL-6 promoters in naive cells, whereas significantly reduced p65 binding at these promoters were observed in BLP-tolerised cells. Notably, overexpression of IRAK-1 promoted NF-kB p65 binding to both TNF-a and IL-6 promoters, thus abrogating BLP tolerisationattenuated NF-kB p65 nuclear transactivation and resulting in an increased proinflammatory cytokine production in BLP-tolerised cells ( $p<0.01$ versus empty vector transfected, BLP-tolerised cells).

Our data highlight a crucial role for IRAK-1 in BLP-induced tolerance and suggest the therapeutic potential of targeting this molecule during bacterial infection.

Conflict of interest: None

Disclosures: None

\section{SESSION 2: PRIZE SESSION}

\section{PROGNOSTIC SIGNATURE IN PANCREAS CANCER}

M.J.F. Smith, A. Rogers, J.F. Murphy, A. McDermott, N. Swan, P. Crotty, P.F. Ridgway, K.C. Conlon

The Professorial Surgical Unit, Trinity College Dublin, Ireland, The Trinity Centre for Health Sciences, AMNCH, Tallaght, Dublin 24, Ireland

Pancreatic cancer is one of the most lethal solid tumours of the gastrointestinal tract with incidence correlating closely to mortality rate (386 cases in Ireland in 2005, 379 deaths), and a 5-year survival rate of $5 \%$. Future improvements in pancreatic cancer therapy are likely to be molecularly based. We performed a pangenomic analysis of human metastatic (AsPC-1) versus non-metastatic (BxPC-3) pancreatic cancer cells generating a "Putative Prognostic Signature" for validation in biobanked invasive pancreatic ductal and ampullary carcinoma specimens. 
AsPC-1 and BxPC-3 were treated with phorbol-12-myristate-13acetate for $4 \mathrm{~h}$ (PMA, pro-inflammatory). Viability assays were conducted using MTT. Biotin-labeled cRNA was hybridised to Affymetrix U133 Plus 2.0 arrays. Differentially expressed genes were validated by quantitative reverse transcription-polymerase chain reaction (qRT-PCR) based on pathway significance and fold change. Expression profiles of resected patients were investigated using the 12-gene putative prognostic signature (including Matrix Metalloproteinase-1, Interleukin-8, Urokinase Plasminogen Activator and Vascular Endothelial Growth Factor). Data was analysed versus control (adjacent normal pancreas, duodenum) and normalised to $18 \mathrm{~S}$ RNA.

Viability assays confirmed the resistant nature of AsPC-1 to PMAinduced cytotoxicity. Microarray investigations profiled cell death via increased extrinsic apoptotic gene expression through death receptors Fas, DR3, DR4-5. All 12 genes were $\geq$ tenfold up-regulated in the more metastatic AsPC-1 cells. Furthermore, the 12 gene-set was highly expressed (10-100-fold) in invasive, metastatic pancreatic ductal and ampullary carcinomas with minimal increase in benign tissues.

We identified a putative prognostic signature that is associated with metastatic tendency. The data represent targets for pancreatic cancer therapy and prognostication.

Conflict of interest: None

Disclosures: None

\section{ACE UP THE SLEEVE-ARE VASCULAR PATIENTS MEDICALLY OPTIMIZED?}

\author{
A.P. Coveney, E.M. Quinn, P. Cronin, G.C. O'Brien, G. Fulton \\ Departments of Academic and Vascular Surgery, Cork University \\ Hospital, Wilton, Cork, Ireland)
}

This prospective study examines the medical management of arteriopathic patients attending a vascular surgical service at a university hospital over a 6 month period. These arteriopathic patients are at significantly higher risk of cardiovascular death compared to healthy controls of similar age. (1) ACE-inhibitors have been shown to reduce the cardiovascular morbidity and mortality rates in patients with peripheral vascular disease by $25 \%$ regardless of the presence or absence of hypertension. (2) Between January and June 2009 data was recorded on sequential patients with arterial disease attending the vascular surgical service. Patients' demographics, type of arterial disease, medical consultations within the previous 12 months and current medications were recorded. The study included 180 patients with a mean age of 69 (39-88). All but $4 \%$ were taking an anticoagulant or antiplatelet, predominantly aspirin. $86 \%$ were taking a statin and $44 \%$ were taking a beta-blocker. $51 \%$ were taking an ACE inhibitor. Suboptimal prescription of ACE inhibitors and betablockers was evident regardless to the types of medical consultations in the previous year. No specialty group differed significantly from vascular surgeons in their prescribing pattern.

Whilst almost all arteriopaths receive some form of anticoagulant and statin in line with clinical evidence, ACE-inhibitors and betablockers appear to be underprescribed in this arteriopathic population. We conclude that opportunity exists for vascular surgeons to embrace recent guidelines and lead the way in both surgical and medical optimisation of arteriopathic patients.

Reference

(1) Criqui MH, Langer RD, Fronek A, Feigelson HS, Klauber MR, McCann TJ et al. (1992) Mortality over a period of 10 years in patients with peripheral arterial disease. $\mathrm{N}$ Engl J Med 326(6):381-386
(2) Yusuf S, Sleight P, Pogue J, Bosch J, Davies R, Dagenais G (2000) Effects of an angiotensin-converting-enzyme inhibitor, ramipril, on cardiovascular events in high- risk patients. The Heart Outcomes Prevention Evaluation Study Investigators. N Engl J Med 342(3):145-153.

Conflict of interest: None

Disclosures: None

\section{NEO-ADJUVANT THERAPY FOR RECTAL CANCER: EXAMINING THE PATHOLOGICAL RESPONSE}

\author{
E. O’Halloran, A.M. Hogan, F.Elgillani, K.J. Breen, K. Mealy
}

Department of Surgery, Wexford General Hospital

The management of rectal cancer has been revolutionised over the last decade. Neo-adjuvant therapy followed by complete mesorectal excision for appropriately staged rectal cancer is now considered the international standard of care. The recognised rate of complete pathological response at time of resection is approximately $20 \%$. The aim of the present study is to examine the post-neoadjuvant response interms of pathological staging and to evaluate local recurrence rate in patients in Wexford General Hospital. To this end a prospectively maintained database of all patients treated for rectal cancer over a 4 year period (2006-2009) was interrogated. A standard radiotherapy regime of 45 Gy to the pelvis in 25 fractions and chemotherapy of 5FU $225 \mathrm{mg} /$ $\mathrm{m}^{2}$ in two cycles intravenously was administered. The rate of complete pathological response was assessed and TNM stage was enumerated. Local disease recurrence over the time period was then investigated. 71 patients (54 male; 17 female) aged 33-81 (median 72) treated for rectal cancers were identified. Clinical staging was by colonoscopy $+\mathrm{CT}+\mathrm{MRI} \pm \mathrm{PET}$ CT and ranged from T2N0M0 to T4N2M0. Postoperative assessment of pathological specimens resulted in complete pathological response (ypT0NOM0) in only three patients $(4.2 \%)$. Of the remainder $58(82 \%)$ were disease free over the study period. Disease recurrence occurred in $13(18 \%)$ patients all of whom had nodal disease at presentation and $92 \%$ had locally advanced disease. In conclusion complete pathological response of rectal cancer to neoadjuvant therapy appears disappointing in our unit despite close adherence to best practice guidelines.

Conflict of interest: None

Disclosures: None

\section{EXPERIMENTAL RUPTURE OF REALISTIC ABDOMINAL AORTIC ANEURYSMS: CAN RUPTURE LOCATIONS BE PREDICTED NUMERICALLY?}

\section{B.J. Doyle, A.J. Cloonan, M.T. Walsh, T.M. McGloughlin}

Centre for Applied Biomedical Engineering Research (CABER), Department of Mechanical and Aeronautical Engineering, and Materials and Surface Science Institute, University of Limerick, Ireland

Although there are arguments as to the most suitable time to repair an abdominal aortic aneurysm (AAA), with maximum diameter remaining the gold standard in terms of rupture risk, there is a substantial amount of work suggesting that diameter does not influence rupture to the extent as once believed. A previous report by this 
author1 highlighted that idealised AAA geometries do not rupture at the region of maximum diameter but rather at regions of inflection at the proximal and distal locations of the AAA sac. This paper furthers this technique to realistic AAA geometries determined from patientspecific AAA cases and created using 2 grades of previously reported silicone rubber. 2 Realistic experimental AAA models ruptured at regions of inflection and complex curvature and not at regions of maximum diameter. Wall thickness of the models was $2.19 \pm$ $0.40 \mathrm{~mm}$, with a wall thickness significantly lower $(p=0.006)$ at the location of rupture $(\sim 1.8 \mathrm{~mm})$. There was however no significant relationship between the rupture pressure and the wall thickness at the rupture site $(p=0.688$ and $p=0.881)$. Computational models accurately predicted the locations of rupture. Rupture occurred at the location of peak and high wall stress in $90 \%(18 / 20)$ of cases. $10 \%(2 /$ 20) of models had defects in the AAA which moved the rupture location away from regions of elevated stress. These defects can be compared to aortic blebs and regions of localised hypoxia in vivo. This paper may help better understand the locations of AAA rupture in patient-specific geometries.

The authors have no conflict of interest.

\section{PRE-CLINICAL EVALUATION OF THE PROLONG ${ }^{\circledR}$ VASCULAR GRAFT}

\author{
G.T. Carroll ${ }^{1}$, T.M. McGloughlin ${ }^{1}$, P.A. Grace ${ }^{2}$, M.T. Walsh ${ }^{1}$ \\ Centre for Applied Biomedical Engineering Research (CABER), \\ Department of Mechanical and Aeronautical Engineering, and the \\ Materials and Surface Science Institute, University of Limerick, \\ Limerick, Ireland ${ }^{I}$, Department of Vascular Surgery, HSE Mid- \\ Western Regional Hospital, Limerick, Ireland ${ }^{2}$
}

The Prolong ${ }^{\circledR}$ Vascular Graft was developed for femoral-popliteal bypass surgery. Traditionally, such a procedure is performed using a straight bypass graft, consisting of two end-to-side graft-artery anastomoses. However, the long-term patency rates of such prosthetic bypass grafts remains low, with 3-year primary patencies of approximately $40 \%$ (1). The distal junction is the primary location of disease formation, resulting from abnormal haemodynamics, material mismatch and surgical injury. The Prolong ${ }^{\circledR}$ graft has a Y-shaped end-to-end distal junction designed to promote rapid flow recovery and eliminate the disease prone bed and therefore reduce disease development in the host artery.

The Prolong ${ }^{\circledR}$ graft was manufactured using commercially available $6 \mathrm{~mm}$ diameter crimped Dacron-graft material and was ETO sterilised. The pre-clinical trial used an ovine model to evaluate the Prolong ${ }^{\circledR}$ device in vivo. The Prolong ${ }^{\circledR}$ graft was implanted to replace the left carotid and each animal served as its own control. The response variables included incidence of graft patency and degree of remodelling within the vascular graft as determined by histologic examination. Eight animals were tested, six animals survived to the predefined survival time (90, 180 and 240 days) and two expired unexpectedly at 2 and 25 days. At time of sacrifice, the Prolong ${ }^{\circledR}$ graft showed an $84 \%$ patency rate compared to $50 \%$ in the control group. Furthermore, histology showed less intimal hyperplasia in the test samples compared to the straight graft. The Prolong ${ }^{\circledR}$ graft has been further developed for use in the dialysis vascular access application and is currently under preclinical evaluation using a porcine model.

(1) O'Brien et al. (2005) Computational investigations of a new prosthetic femoral-popliteal bypass graft design. J Vasc Surg 42:6, 1169-1175.

Conflict of interests: None

Disclosures: None

\section{XENOGENIC EXTRACELLULAR MATRICES AS POTENTIAL BIOMATERIALS FOR FUNCTIONAL RECONSTRUCTION OF THE LOWER GENITOURINARY TRACTS}

\author{
N.F. Davis ${ }^{1,2}$ A. Callanan ${ }^{2}$, B.B. McGuire ${ }^{1}$, H.D. Flood ${ }^{1}$, \\ T.M. McGloughlin ${ }^{2}$
}

Department of Urology, Mid-Western Regional Hospital, Limerick ${ }^{I}$, The Centre for Applied Biomedical Engineering Research, University of Limerick, Castletroy, Co. Limerick, Ireland ${ }^{2}$

Introduction: Reconfigured autogenous bowel is a treatment option for functional reconstruction of the lower genitourinary tracts. Concerns persist as the intestine is lined with absorptive mucus-secreting epithelium that is not ideal in a urinary reservoir. Functional reconstruction with a biocompatible acellular xenogenic material may be an alternative option. This study compares synthetic materials with the regenerative and remodelling capabilities of xenogenic extracellular matrices (ECMs) when seeded with human urothelial cells.

Methods: Human urothelial cells were cultured onto synthetic polypropylene, porcine small intestinal submucosa (SIS) and porcine urinary bladder matrix (UBM) under identical growth conditions and seeding densities of 20,000 cells $/ \mathrm{cm}^{2}$. Both luminal and abluminal surfaces of the SIS and UBM bioscaffolds were tested for proliferation and cellular viability using fluorescent markers for cytoplasm and nuclei. Differentiation and growth rate was assessed by Almer blue and MTT assays.

Results: Cellular proliferation and growth rate varied on luminal and abluminal surfaces of UBM and SIS bioscaffolds. Greater proliferation rates indicated preferred substrate architecture and improved growth performance of the urothelial cells. Cell growth and proliferation was superior on UBM and SIS substrates compared to synthetic grafts. Longer seeding periods (6 days) showed the highest proliferation and growth rates. An intact basement membrane within UBM (luminal surface) caused cell monolayer formation, without any surface infiltration.

Conclusion: Xenogenic UBM possesses more favourable characteristics that favour genitourinary tract application in comparison to other xenogenic and synthetic ECMs. Its intact basement membrane may prevent urine infiltrating into deeper tissues while supporting growth and differentiation of human urothelial cells.

Conflict of interest: None

Disclosures: None

\section{EXPRESSION OF MICRORNAS AND THEIR PREDICTED PROTEIN TARGETS IN THE CIRCULATION OF BREAST CANCER PATIENTS: NOVEL BIOMARKERS FOR BREAST CANCER}

\section{R.J. Kelly, H.M. Heneghan, N. Miller, M.J. Kerin}

Department of Surgery, Clinical Science Institute, National University of Ireland, Galway, Ireland

Background: Mi(cro)RNAs hold several characteristics of an ideal tumour marker given that they are dysregulated in cancer, their expression patterns are tissue-specific, and are remarkably stable in tissue and fluid media. Thus circulating miRNAs hold great potential as novel minimally invasive cancer biomarkers. The aims of this study were to investigate for the presence of cancer specific miRNAs in the circulation of breast cancer patients and to examine the relationship between candidate miRNAs and their predicted protein targets. 
Methods: Blood samples were collected prospectively from 133 study participants including 89 consecutive breast cancer cases and 44 healthy age-matched controls. The expression of candidate miRNAs were quantified in peripheral blood samples by RQ-PCR. Levels of predicted protein targets, Caspase- 3 and BCL-2, were quantified by ELSIA in matched serum samples form all participants.

Results: Cancer-specific miRNAs were detectable and altered in the circulation of breast cancer patients, including those with in situ disease. Notably, 2 miRNAs were significantly elevated $(P>0.001)$ in blood from breast cancer patients compared to controls. Postoperative analysis of a subset of patients indicated a significant drop $(P<0.001)$ in miRNA levels. Levels of the predicted protein targets did not correlate with systemic miRNA levels.

Conclusion: Our results demonstrate the novel finding that miRNAs are detectable in the circulation of breast cancer patients and that systemic miRNAs have great potential as novel minimally invasive tools to aid in the early diagnosis and perioperative management of breast cancer. Conflict of interest: None

Disclosures: None

\section{A SNP IN LET-7 MIRNA BINDING SITE IN THE KRAS ONCOGENE INCREASES RISK OF TRIPLE NEGATIVE BREAST CANCER}

\author{
H.M. Heneghan ${ }^{1}$, N. Miller ${ }^{1}$, T. Paranjape ${ }^{3}$, F.J. Slack ${ }^{2}$, J.B \\ Weidhaas $^{3}$, M.J. Kerin ${ }^{1}$
}

Department of Surgery, National University of Ireland, Galway, Ireland $^{I}$, Department of Molecular, Cellular and Developmental Biology, Yale University, New Haven Connecticut ${ }^{2}$, Department of Therapeutic Radiology, Yale University, New Haven Connecticut ${ }^{3}$ )

MiRNAs, small non-coding RNAs, exert their regulatory effect on gene expression by binding to the $3^{\prime}$ untranslated region (UTR) of target genes. The let-7 family of miRNAs are global genetic regulators important in controlling oncogene (e.g. KRAS) expression. Dysregulated let-7 is associated with many malignancies including breast cancer. We hypothesized that a single nucleotide polymorphism (SNP) in the let-7-binding site in the KRAS oncogene (LCS6SNP) disrupts let-7 regulation of KRAS, and thus could increase risk of developing breast cancer.

A population analysis of the association of LCS6SNP and breast cancer was performed on a cohort of 1,010 breast cancer patients and 1,497 age-matched non-cancer controls recruited in the West of Ireland, and New Haven, Connecticut. Genomic DNA isolated from blood samples was amplified using PCR assays designed specifically to identify the T or G allele of the LSC6 SNP. Genotyping results were correlated with patient s' clinicopathological parameters.

The LCS6 variant was present in $15 \%$ in all breast cancer patients, compared with a baseline prevalence of $5.8 \%$ in controls. Interestingly, the SNP was most prevalent $(30 \%)$ in patients with triple negative breast cancer (Basal subtype), and in premenopausal women.

This is the first report of inherited variation in oncogenic miRNAs. The LCS6 variant allele in KRAS $3^{\prime}$ UTR is significantly associated with increased risk of breast cancer in young females, in particular basal subtype; the most challenging breast cancer to treat and which carries a poor prognosis. This represents a new paradigm for breast cancer susceptibility and is a novel biomarker of risk for developing triple negative breast cancer in young women.

Conflict of interests: None

Disclosures: None

\section{THE ADIPOCYTOKINE LEPTIN AND ITS PATHWAY ARE UP REGULATED IN OBESE BREAST CANCER PATIENTS: A PATHWAY FOR OBESITY IN BREAST CANCER}

P.A. Carroll ${ }^{1}$, L. Healy ${ }^{2}$, E. Allot ${ }^{1}$, J. Lysaght ${ }^{1}$, B. Dunne ${ }^{3}$, M. Griffin ${ }^{3}$, T. Boyle ${ }^{1}$, J.V. Reynolds ${ }^{1}$, M.J. Kennedy ${ }^{4}$, G. Pidgeon ${ }^{1}$, E.M. Connolly ${ }^{1}$

Department of Surgery, St. James's Hospital and Trinity College Dublin, Ireland ${ }^{l}$, Department of Clinical Nutrition, St. James's Hospital, Dublin, Ireland ${ }^{2}$, Department of Pathology, St. James's Hospital, Dublin, Ireland, , Academic Unit of Clinical and Molecular Oncology, St. James's Hospital and Trinity College Dublin, Ireland ${ }^{4}$

Introduction: Obesity is an increasingly recognised risk factor for breast cancer. Adipocytokines produced by local mammary adipose tissue (paracrine) as well as distant adipose deposits (endocrine) may be the mechanism by which obesity influences breast cancer.

Aims: To determine the effect of obesity on gene expression of the adipocytokines leptin $(\mathrm{Ob})$ and adiponectin (Adipo) in mammary adipose tissue, and their receptors (ObR, AdipoR1/R2) in tumour tissue.

Methods: Breast cancer patients $(n=40)$ undergoing surgery had anthropomorphic (Tanita BIanalyser, Waist circumference, BMI) and serological measurements of obesity/metabolic status (Glucose, Lipids, Insulin) performed. Matched adipose and tumour tissue were analysed using quantitative real time PCR for mRNA expression of a panel of genes: Ob, Adipo, ObR, AdipoR1/R2.

Results: $70 \%$ women were obese. In mammary adipose tissue, there was upregulation of $\mathrm{Ob}$ in obese women $(p<0.05)$ relative to normal weight controls. Tumour samples expressed all three receptors. Expression of ObR was significantly increased in obese women compared to normal weight cancer patients $(p<0.05)$. Serum leptin tended to positively correlate with ObR expression $(r=0.4$, $p=0.055)$ while serum insulin, a marker of obesity and insulin resistance correlated strongly with $\mathrm{ObR}(r=0.58, p<0.005)$. There was no difference in tumour AdipoR1/R2 or gene expression compared to obesity status.

Conclusion: Obesity status is associated with increased expression of the leptin gene in mammary adipose tissue and with increased leptin receptor expression in tumour tissue. ObR is also positively correlated with serum leptin and insulin. This association suggests the adipocytokine pathway regulates tumour biology. This may link the molecular association of obesity with breast cancer, highlighting potential targets for prevention and treatment strategies.

Conflict of interest: None

Disclosures: None

\section{MOLECULAR CLASSIFICATION OF BREAST CANCER AND RELATIONSHIP TO SURVIVAL}

\author{
M.C. Hartmann, A.M. Kiely, M.C. Whelan, C. Curran and M.J. Kerin
}

Department of Surgery, National University of Ireland Galway, Galway, Ireland

Breast cancer is classified into molecular subtypes identified by gene expression profiling. The aim of this study was to investigate prevalence and survival trends of these subtypes.

Data of patients with invasive breast cancer $(n=1,122)$ were obtained from a prospectively maintained database. Subtypes were classified as Luminal A (ER+ and/or PR+), human epidermal 
growth factor 2 negative (HER-2-), Luminal B (ER+ and/or PR+, HER-2+), HER-2 (ER-, PR-, HER-2+) and Basal (ER-, PR-, HER-2-). The chi-squared test was used to determine the association of subtypes with clinicopathological parameters. Overall survival trends were investigated using Kaplan-Meier product-limit method.

Distribution of subtypes in our study population was Luminal A $70 \%(n=787)$, Luminal B $13 \%(n=147)$, Basal $9 \%(n=103)$ and HER-2 8\% $(n=85)$. Patients with Luminal A subtype were significantly less likely to present with high grade tumour and nodal involvement compared to individuals with Basal or HER-2 subtypes $(p<0.05)$. A statistically significant higher proportion of Luminal B subtype and lower proportion of Luminal A subtype was seen in patients aged 40 years and younger $(n=86)$ compared to older age groups $(n=422, p<0.05)$. The Luminal A subtype was significantly more likely to be lobular invasive carcinoma $(p<0.0001)$. Mean overall survival time in the study population was 28 months. Analysis revealed a trend towards better overall survival for patients with Luminal A compared with Luminal B and Basal subtypes. Log rank tests comparing all subtypes revealed statistically significant differences in overall survival between subtypes $(p<0.05)$.

The observed differences in clinicopathological parameters and survival time between breast cancer molecular subtypes warrant further investigation and represent steps towards individualised breast cancer therapy.

Conflict of interest: None

Disclosures: None

\section{COLUMNAR CELL LESIONS OF THE BREAST: A 5 YEARS EXPERIENCE OF A BREAST UNIT}

\author{
A. Calamai ${ }^{4}$, R. Salman ${ }^{3}$, M. Kennedy ${ }^{1}$, F. Flanagan ${ }^{2}$, T. Gorey ${ }^{3}$, \\ M. Kell ${ }^{3}$, M. Stokes ${ }^{3}$
}

Department of Histopathology ${ }^{1}$, Radiology ${ }^{2}$ and Surgery ${ }^{3}$ and Breast Check and Breast Health, Mater Misericordiae University Hospital, University College Dublin ${ }^{4}$, Dublin, Ireland

The widespread use of mammographic breast screening and the introduction of even more sensitive radiological techniques have placed increasing demands upon the pathologist for the accurate diagnosis and histological categorisation of screen-detected lesions

Columnar cell lesions (CCL) are one of the most common abnormalities in the adult female human breast, characterized by the presence of columnar-shaped epithelial cells lining enlarged terminalduct lobular units.

CCLs with Flat Epithelial Atypia (FEA) are being seen increasingly in biopsies taken for the non-palpable calcifications. The increase incidence may reflect improved delineation and recognition of CCL by pathologist or a true increase in incidence related to biological and/or environmental factors.

The aim is to audit our experience of CCL in core biopsies and to elucidate evolving clinical and diagnostically relevant principles that surround and influence the significance of this controversial entity, especially when discovered on needle core biopsy (NCB) in the initial phase of breast diagnosis and management.

We reviewed all B3/B4 lesions seen on NCB from the symptomatic and the screening population attended our unit between January 2004 and December 2008.

We compared the radiological scoring, the pathological diagnosis of CCL with FEA on NCB and the outcome of the diagnostic open biopsies.

Reviewing our database, a total of 348 cases of B3/B4 lesion identified and 50 cases had showed features of CCL with 14 cases showed FEA; the majority being a screening detected lesion. As per our policy all these cases had an open diagnostic biopsy. 7/14 (50\%) showed an insitu carcinoma.

From our finding we justify our policy of removing all CLL with FEA found on NCB as a significant number had showed an associated carcinoma mainly insitu component.

Conflict of interest: None

Disclosures: None

\section{SESSION 3: HEAD AND NECK}

\section{THE USE OF LYMPHOCYTE TO WHITE CELL COUNT RATIO IN THE DIAGNOSIS OF INFECTIOUS MONONUCLEOSIS}

\author{
P. Lennon, J.P. O’ Neill, J. Fenton, T.P. O’Dwyer \\ Department of Otolaryngology, Mater Misericordiae University \\ Hospital, Eccles St., Dublin 7, Ireland
}

We investigated the hypothesis that a Lymphocyte/White Cell Count (L/WCC) ratio should be used as a diagnostic indicator of Infectious Mononucleosis. We carried out a retrospective study to compare lymphocyte counts and white blood cell counts, against the criterion standard, the mononucleosis spot tests. This took place in the Department of Otolaryngology, Mater Misericordiae University Hospital, Dublin, Ireland. We reviewed 1,000 patients who had monspot assays, 500 positive and 500 negative. The L/WCC ratio was calculated and compared with the monospot result, in order to calculate the sensitivity and specificity of a ratio of 0.35 . The L/WCC ratio was significantly different in the two groups $(P<0.05)$. The mean L/WCC ratio in the positive group was 0.49 and the mean L/WCC ratio in the monospot negative group was 0.29 . For the detection of glandular fever a ratio higher than 0.35 had a specificity of $72.2 \%$ and a sensitivity of $84 \%$. A higher ratio will give a greater specificity, but a lower sensitivity, and visa versa. In conclusions the lymphocyte to white cell count alone is not sufficient to diagnose or exclude Infectious Mononucleosis.

Conflict of interest: None

Disclosures: None

\section{TOTAL THYROIDECTOMY: PREDICTION OF HYPOCALCAEMIA BY POST-OPERATIVE PARATHORMONE MEASUREMENT}

\author{
D. Fitzgerald, C.Timon \\ Royal Victoria Eye and Ear Hospital, Adelaide Road, Dublin 2, \\ Ireland
}

The objective of the study is to assess postoperative Parathormone (PTH) level as a predictor of hypocalcaemia, and thus prolonged hospitalisation, in patients who have undergone total thyroidectomy.

This prospective study was undertaken at a university teaching hospital, dedicated to Otolaryngology, Head and Neck Surgery.

We studied forty patients, over a 9 month period, who underwent total or completion thyroidectomy.

Complete data was obtained for the patients, including PTH measurement in the immediate post-operative period. Patients were monitored for hypocalcaemia. 
Results showed all patients with post-operative PTH $>15$ pg/ml remained biochemically and clinically normocalcaemic.

In conclusion, PTH, when measured $4-6 \mathrm{~h}$ post-operatively has excellent, statistically significant, accuracy in determining which patient will become hypocalcaemic, allowing for potential patient discharge to be achieved earlier. PTH measurement may potentiate more cost effective total thyroidectomy.

Conflict of interest: none

Disclosures: none

\section{FINITE ELEMENT ANALYSIS MODEL DEVELOPMENT FOR AN EDENTULOUS ATROPHIC MANDIBLE: THE BUCKET HANDLE FRACTURE}

\author{
E.P. Kavanagh ${ }^{1}$, F. Wallis ${ }^{2}$, T. McGloughlin ${ }^{1}$ G. Kearns ${ }^{3}$, J. Jarvis ${ }^{4}$ \\ Centre for Applied Biomedical Engineering Research (CABER), \\ University of Limerick, Ireland ${ }^{l}$, Department of Radiology, Mid- \\ Western Regional Hospital, Limerick, Ireland ${ }^{2}$, National \\ Maxillofacial Unit, St. James Hospital, Dublin, Ireland ${ }^{3}$, Department \\ of Mechanical and Aeronautical Engineering, University of Limerick, \\ Ireland ${ }^{4}$
}

Oral and Maxillofacial Surgeons (OMFS) use various methods of plate and screw fixation in the treatment of mandibular fractures. The edentulous atrophic mandibular fracture, in which the teeth are absent and the alveolar ridge resorbed, presents a significant clinical challenge. The precise location of the internal fixation plates and the specific methods of treatment require consideration.

An uncommon fracture that occurs in an edentulous atrophic mandible is known as a "bucket-handle" fracture. This is a bilateral mandibular body fracture occurring at the thinnest portion of the atrophic mandible. It results in significant displacement of the anterior mandible in an inferior direction due to the action of the suprahyoid muscles and displacement of the posterior mandibular body in a superior direction due to the action of the ptergomasseteric sling. This is a rare but complex fracture associated with a high incidence of post operative complications. Therefore, controversy exists as to the ideal treatment in this clinical situation.

In the atrophic mandible the blood supply is primarily derived from the periosteum. Placement of bone plates on the lateral aspect of the mandible requires stripping of the periosteum and potential compromise of the blood supply. However, locating the plate on the inferior border may avoid periosteal stripping but may be biomechanical unsound as show in previous studies 1 .

To address this clinical problem from a biomechanical standpoint, a model was constructed using a CT scan of a 70 year old female patient with an edentulous atrophic mandible. This was constructed using the Mimics software programme and imported into Finite Element Analysis software Abaqus where mechanical properties were assigned and fracture points inserted. The aim of this study is to progress the healthy mandible model to include an edentulous atrophic mandible and to resolve the question of how best to deal with these uncommon fractures from a biomechanical standpoint and provide important information for the clinician.

(1) Kavanagh EP, Frawley C, Kearns G, Wallis F, McGloughlin T, Jarvis J (2008) Use of finite element analysis in presurgical planning: treatment of mandibular fractures. Int $\mathrm{J}$ Med Sci 177(4):325-331

Conflict of interest: None

Disclosures: None

\section{IS THERE A CORRELATION BETWEEN INTRAOPERATIVE HYPOTHERMIA AND POSTOPERATIVE MORBIDITY IN HEAD AND NECK CANCER PATIENTS?}

\author{
R. Mehanna, J.P. O’Neill, C. Timon \\ Otorhinolaryngology Head and Neck Surgery Department, St. James \\ Hospital, Dublin 8, Ireland
}

It is well documented that decreased core temperature leads to decreased blood flow causing reduced postocclusive reactive hyperaemia and increased vascular resistance in the flap. We hypothesized this leads to an increased risk of flap complications.

This study aimed to establish if intraoperative hypothermia correlated with increased postoperative morbidity in patients undergoing resections of head and neck cancers with subsequent free/pedicled flap(s) reconstruction.

A retrospective chart review for patients undergoing flap reconstruction in our institution (academic tertiary care hospital) over 5 years was performed. Several patient variables were analyzed including age, sex, oncological stage, smoking status, and prior radiotherapy. Intraoperative temperature was measured via urinary catheter, and a mean calculated. The results were analyzed using independent $t$ test, odds ratio, and logistics regression.

Of the 41 patients included, 16 had pedicled/rotational flaps, 23 had free flap reconstructions, and 2 underwent a combination of free and rotational flaps. Of these patients, 15 experienced flap complications with $9.5 \%$ having greater than $50 \%$ flap loss/dehiscence. And 9.5\% with less than $50 \%$ flap loss. Two patients developed myocutaneous fistulas. It was calculated that with a $1{ }^{\circ} \mathrm{C}$ temperature drop, the probability of having postoperative complications increased by 6.43 times.

In conclusion, hypothermia is known to cause reduced blood flow and increased vascular tone. This causes significant postoperative flap morbidities. Other variables such as age, smoking and previous radiotherapy had no impact on morbidity. Thus, hypothermia is an independent risk factor for the development of postoperative morbidity in patients undergoing flap reconstruction for head and neck cancers.

Conflict of interest: None

Disclosures: None

\section{INVESTIGATING THE EFFECT OF TONSILLECTOMY ON VOICE}

\section{C.B. Heffernan, M.A. Rafferty}

The Royal Victoria Eye and Ear Hospital, Adelaide Road, Dublin 2, Ireland

Anecdotal evidence suggests that tonsillectomy may alter voice. Any variation may not be apparent in the speaking voice but may impact on the professional voice user. The first and second formants (F1 and F2) determine the quality of the speaking voice. Controversy remains over the importance of the higher formants (F3 and F4). F4 is often referred to as the singer's formant as its presence is felt to improve the harmonic quality of the voice.

We designed a prospective study to investigate the formant alterations after tonsillectomy.

All adults undergoing tonsillectomy from July to December 2009 were enrolled. Prior to tonsillectomy formant values were estimated. At surgery measurements were taken of the pharynx 
before and after dissection.Tonsil weight was recorded. The acoustical measurements were repeated on the first post-operative day and again 4 weeks later.

Twenty-six patients were enrolled in the study (19 female; 7 male). The age range was $15-40$ years. On average F1 rose, F2 slightly rose and F3 slightly declined without any appreciable loss of vocal tract steadiness. Pre-operatively $58 \%$ of patients had a consistent F4 and post-operatively $88 \%$ of patients had a consistent F4 (Fig. 1).

Tonsillectomy has no significant impact on the parameters of normal speech. However the singer's formant is present more frequently in the post-operative recordings. Therefore it would appear that F4 is enhanced by increasing the horizontal dimensions of the oropharynx. This suggests that the post tonsillectomy voice may have a superior quality.

Conflict of interest: None

Disclosures: None

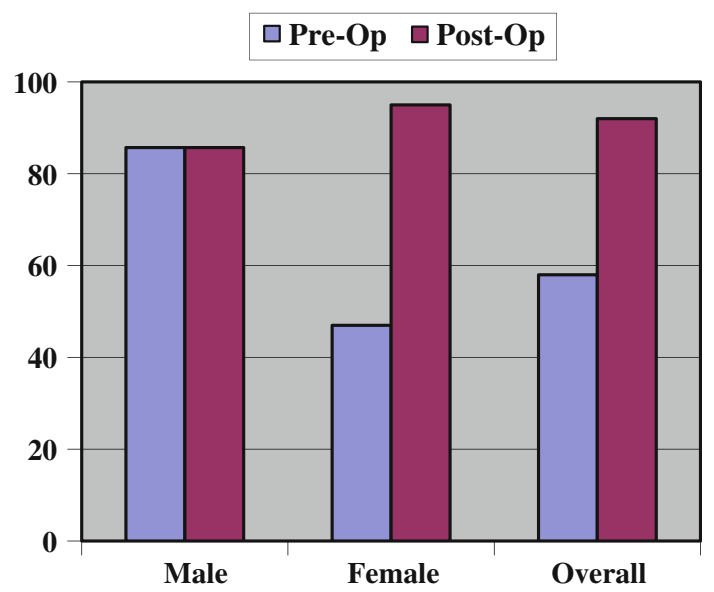

\section{A PROSPECTIVE TRIAL OF PAIN AFTER MINIMALLY INVASIVE VIDEO ASSISTED VERSUS MINIMALLY INVASIVE OPEN THYROIDECTOMY}

\author{
J.P. O’Neill, C. Timon \\ Department of Otolaryngology, Head and Neck Surgery, \\ St James Hospital, Dublin, Ireland
}

Background: The analysis of this prospective study was to compare pain score results in patients who underwent minimally invasive video-assisted (MIVAT) versus minimally invasive open thyroidectomy (MIT) and in doing so further extrapolate the potential advantages of video-assisted thyroid surgery.

Methods: A prospective trial of 98 patients post minimally invasive thyroidectomy from January 1st to December 31st 2008 are the subject matter of this study. Pain scores $(0-10)$ assigned by the patients shortly after the thyroidectomy and after $24 \mathrm{~h}$ post-operative forms the basic outcome variable. For statistical comparison of the distributions of the pain scores between patients the KolmogrovSmirnov test was employed.
Results: The comparison pain score distribution using KolmogorovSmirnov test for post operative assessment yielded a $Z$ value of 2.84 $(P<0.001)$. The comparison pain score distribution at $24 \mathrm{~h}$ yielded a $Z$ value of $1.48(P<0.05)$. These results imply the difference in distributions of pain scores among MIT and MIVAT group to be statistically significant.

Conclusion: Video-assisted thyroidectomy appears to reduce the pain in patients shortly after the operation and $24 \mathrm{~h}$ post-operative. The reduction is statistically significant. Male patients appear to report less pain compared to their female counter parts.

Conflict of interest: None

Disclosures: None

\section{MANAGEMENT OF PAROTID STONE CALCULUS}

D. Galvin, A. Curran

Royal Victoria Eye and Ear Hospital, Adelaide Road, Dublin 2, Ireland

Introduction: Parotid gland calculi, although less common that their submandibular counterparts pose a management problem. Removal of the stones is difficult without causing iatrogenic facial nerve palsy. Case history: MD is a 72 year old lady presenting with a long-term history intermittent swelling of the left parotid gland. She was referred to ENT with a 6 month history of persistently swollen left parotid gland. A large impacted calculus imbedded in the parotid was the cause. Removal of the stone was difficult. A failed attempt at sialendoscopy made an combined approach the only option.

Conclusion: Although sialendoscopy has revolutionised the management of salivary gland calculi, large impacted or multiple stones are not amenable to minimally invasive removal.

Conflict of interest: None

Disclosures: None

\section{COMPLICATIONS OF COCHLEAR IMPLANT SURGERY: BEAUMONT HOSPITAL, DUBLIN 1995-2008}

\author{
D.R. Gough, A. O'Connor, P. Walshe, L. Viani
}

National Cochlear Implant Centre, Beaumont Hospital, Dublin, Ireland

In a retrospective analysis, we evaluated the intra and post-operative complications in patients who underwent cochlear implantation between 19995 and 2008 at the National Cochlear Implant Centre, Beaumont hospital, Dublin.

The study involving 371 patients, is the first of its kind since the programme's inception in 1995. Relevant parameters included intra, early and late post-operative complications such as device failure, early and late infection. The complication rate compares favourably with best international standards. Furthermore, complications that require surgical intervention can be treated effectively without long term morbidity.

Conclusion: Our data indicates that cochlear implant surgery, in both adults and children is a safe and reliable procedure with a low complication rate.

Conflict of interest: None

Disclosures: None 


\section{ELECTRONIC FOLLOW UP OF LACERATION IN THE EMERGENCY DEPARTMENT}

\author{
T.W. M. Walker ${ }^{1}$, S. Byrne ${ }^{2}$, P.J. McCann ${ }^{3}$ \\ Department of Oral and Maxillofacial Surgery, Mid-Western \\ Regional Hospital, Limerick, Ireland ${ }^{1}$, National University of Ireland \\ Galway, Ireland ${ }^{2}$, Department of Oral and Maxillofacial Surgery, \\ University Hospital, Galway, Ireland ${ }^{3}$
}

Introduction: It is difficult for a emergency medicine staff to followup on the primary closure of traumatic facial lacerations. This is important to allow closure of the feedback loop for the trainee and also to allow the patient to gain access to revision surgery should this be necessary.

With the advent of mobile phones with cameras, and computers with "web cams" or digital cameras in many homes, we decided to examine the role of electronic follow up of facial lacerations in the emergency department.

Methods: Fifty consecutive patients who were sutured by a single operator using the same technique who had internet access and a digital camera or a mobile phone with a camera were asked to send a photograph of the laceration at 6 and 12 months. A leaflet with the details of the email address or mobile number to send the picture was given.

Patients were given a reminder telephone call at 6 and 12 months and the email address or mobile number to send the picture was re given.

At each phone call patients were asked to grade the healing on a simple scale; 1-very happy, 2-happy, 3-okay, 4-unhappy, 5very unhappy.

At 13 months all patients were re contacted and reminded again. A month was given after each phone call for patients to return a photograph. After this time the photo was designated as "late".

Results: No patient returned a photograph at 7,13 or 14 months. However, all patients had stated they would return a photo. All patients were happy or very happy with the result. No patients wanted referral for scar revision by 13 months. The male: female ratio was 1.94:1. The mean age was 27.2. Most injuries were caused by assault. Twenty-four patients said they had access to a computer/camera to send in the image, 26 patients had a phone with a camera. At 6 months, 23 patients were happy and 27 were very happy with the scar.

Conclusion: There is no evidence to support the use of emailed or text messaged photographs, despite telephone reminders, for the follow up of facial lacerations sutured in the emergency department. It would appear that despite technological advances there is no simple way for closing the feed back loop in this situation.

Conflict of interest: None

Disclosures: None

\section{AND THE WINNER IS...-A SCORING SYSTEM FOR GRADING SURGICAL ORAL PRESENTATIONS}

\section{B.G. Fennessy, J Saunders, J.E. Fenton \\ Department of ENT, Mid-Western Regional Hospital, Limerick, Ireland}

Introduction: In general, surgical trainees are expected to undertake and present research at national and international meetings. Typically, the prizes at the meetings are awarded based on the evaluations of adjudicating chairmen; no generic scoring system has been developed to appraise presentations.
Having performed a pilot study previously, the aim of our study was to develop a presentation scoring system, and incorporate it into a national ENT meeting in an attempt to select a winner. We also assessed if there was any difference between how the consultant and non-consultant hospital doctors graded the presenters.

Methods and materials: Registrar presentations at the Royal Academy of Medicine in Ireland ORL HNS section were evaluated, using the headlines introduction, methods, results, discussion, conclusion, presentation style, data presentation, time keeping, ability to answer questions and scientific merit. Each was scored on a scale of 1 to 5 . Results: There were a total of 28 assessors ( 15 consultants, 8 NCHDs and 5 not known) appraising 18 presenters. Most of the time the consultants were in agreement with the NCHDs but there were differences. In addition, NCHDs tended to score higher than consultants. Further analysis is pending.

Discussion: As far as we can ascertain in a review of the literature, a generic scoring system for oral surgical presentations has not been published previously. We present a feasible, easy to use grading system, which may prove a reliable means by which a winning presenter can be chosen over contemporaries.

Conflict of interest: None

Disclosures: None

\section{FUNCTIONAL SURGERY USING CONSCIOUS SEDATION FOR TEMPOROMANDIBULAR JOINT EMINECTOMY PROCEDURES: A CASE SERIES}

\author{
M. O’Halloran, L.F.A. Stassen \\ National Maxillofacial Unit, St. James's Hospital, Dublin 8, Ireland
}

Purpose: The purpose of this case series study was to evaluate the advantages and disadvantages of using local anaesthesia and conscious sedation (CS) as opposed to general anaesthesia (GA) for performing eminectomy procedures on patients suffering from temporomandibular joint (TMJ) closed lock. Performing this procedure using CS allows functional assessment of the TMJ meniscus at the time of surgery. The objectives of this study were to determine if this functional surgery is beneficial and also to investigate the patients' acceptability for this treatment.

Patients and methods: Twenty-two patients who underwent this eminectomy procedure either under local CS or under GA on a daycase basis participated in this retrospective case series study. All the recruited patients had this procedure carried out following a course of non-surgical conservative treatment which had failed to relieve the symptoms associated with their TMJ disorder.

Objective results obtained were: Pre-operative and post-operative mouth opening

Subjective results obtained were: Pre-operative and post-operative pain and problems Patient rating of operation success

Results: Paired $t$ testing showed a significant change in mouth opening for both groups. The mean mouth opening change in the CS group $(n=9)$ was $10.6 \mathrm{~mm}(p=0.014)$ and in the GA group $(n=13)$ this was $13.7 \mathrm{~mm}(p<0.001)$. An unpaired $t$ test showed there is no statistical difference between these groups $(p=0.467)$. The subjective data recorded showed comparable outcomes and acceptance for the two techniques.

Conclusions: The use of local anaesthesia and conscious sedation seems to be a reasonable alternative to general anaesthesia for performing eminectomy procedures in appropriately selected patients.

Conflict of interest: None

Disclosures: None 


\section{SESSION 4: CLINICAL}

\section{AN AUDIT OF PERIPHERAL VENOUS CATHETER USE IN SURGICAL PATIENTS: MORE WORK TO BE DONE}

\author{
S. McHugh ${ }^{1}$, S. Cowman ${ }^{2}$, S. Tierney ${ }^{1}$, A.D.K. Hill ${ }^{1}$, H. Humphreys ${ }^{3}$ \\ Department of Surgery, Royal College of Surgeons in Ireland ${ }^{l}$, \\ Department of Nursing, Royal College of Surgeons in Ireland ${ }^{2}$, \\ Department of Microbiology, Royal College of Surgeons in Ireland ${ }^{3}$
}

Introduction: Catheter-related blood stream infections (CRBSI) account for $7 \%$ of all healthcare associated infections, and peripheral venous catheters account for $35 \%$ of these. In 2007 the estimated cost per CRBSI was between $\$ 34,000$ and $\$ 56,000$, resulting in an additional hospital stay of 7 days. The duration of cannulation is a major risk factor for CRBSI, with the National Nosocomial Infections Surveillance (NNIS) system in the U.S.A. reporting a CRBSI rate of 5.7 per 1,000 catheter days. We audited peripheral venous cannulae usein an Irish teaching hospital to compare practices with accepted international standards.

Methods: Specific data collection sheets were developed. Patients and medical notes on surgical wards were examined and data collected. Data scanning technology was utilized to transfer information to excel worksheets thus minimizing human error.

Results: 272 peripheral venous cannulae were assessed on 5 surgical wards over a 6 month period. $87.1 \%$ were appropriately maintained with clean dressings, however $10.6 \%$ were in situ for longer than the recommended 72 h. $38.2 \%$ of peripheral cannulae were no longer being used but remained in situ. Of these unnecessary cannulae, the most common reason for being originally sited was for venous access pre-operatively.

Conclusions: This audit highlights important deficiencies in our care of peripheral intravascular catheters. We will now seek to enforce best practice on the duration of peripheral cannulae use and early removal when not in use as part of a post-operative checklist in theatre.

Declarations: Disclosure: Seamus McHugh is funded as a research fellow by the Royal College of Surgeons in Ireland.

The authors declare no conflict of interest.

\section{CHANGING DEMOGRAPHICS IN PRESENTATION FOR LAPAROSCOPIC CHOLECYSTECTOMY}

\section{J. Ryan, J. Broderick, S.T. Martin, G.P. McEntee}

Department of Hepatobiliary Surgery, Mater Misericordiae University Hospital, Dublin, Ireland

Introduction: Laparoscopic cholecystectomy is a common general surgical procedure. The increasing incidence of obesity may have sequelae for patients undergoing surgery for symptomatic gallstone disease.

Aim: Retrospective analysis of operations for gallstone disease at our institution over a 15-year period was performed to establish a trend in patient weight and to analyse corresponding sequelae.
Methods: Data was collected from HIPE-database, histopathology/ theatre records and chart review.

Results: 1,200 cases (758 female) were randomly selected for analysis over a 15 -year period. During each 5-year period (19931997, 1998-2002, 2003-2007) the median patient age decreased 53, 52 and 50 years, respectively. Male patients were more likely to require pre-operative hospitalisation (39 vs. 19.8\%, $p=0.000$ ), post-operative admission ( 16.7 vs. $5.7 \%, p=0.01)$, to have a longer hospital stay (LOS) (5.5 vs. 3.3 days, $p=0.003)$ and were more likely to have a post-operative complication (24 vs. $9.9 \%$, $p=0.000)$ and require subsequent re-intervention (9.3 vs. $1.7 \%$, $p=0.000)$. An increase in female mean weight was observed between the study periods 1993-1997 and 2003-2008 (69.3 vs. $83 \mathrm{~kg} p=0.031)$. Women with weight $>90 \mathrm{~kg}$ had significantly higher (X6) intraoperative complications, $p=0.024$. An overall decrease in post-operative LOS for the entire study period was also noted $(p=0.023)$.

Conclusion: The weight of female patients undergoing laparoscopic cholecystectomy has increased. Female patients over $90 \mathrm{~kg}$ have a sixfold increased risk for intra-operative complications. Male patients are more likely to require pre-operative hospitalisation, post-operative admission, to have a longer LOS and have a post-operative complication/require subsequent re-intervention.

Conflict of interest: None

Disclosures: None

\section{LAPAROSCOPIC RESECTION FOR COLORECTAL NEOPLASIA-A SINGLE CENTRE EXPERIENCE OF 342 PATIENTS}

D.W. Good, J.M. O'Riordan, D. Moran, E. Guare, F.B. Keane, D.S. O'Riordain, P. Neary

Department of Colorectal Surgery, AMNCH, Tallaght, Dublin 24, Ireland

Introduction: Reluctance to embrace laparoscopic surgery for colorectal neoplasia still exists and stems from concerns regarding oncological safety and the learning curve required.

Aim: To determine the short-term outcomes for patients undergoing laparoscopic colorectal surgery for neoplasia.

Methods: Patients undergoing surgery for colorectal neoplasia from June 2005 to September 2009 were reviewed using a prospective colorectal cancer database.

Results: Three hundred and forty-two patients had surgery, 314 patients had laparoscopic surgery, 48 required conversion to open (conversion rate $15 \%$ ) and 28 were planned open. Median node retrieval for laparoscopic was 12 (9-15) and open was 12 (8.75-16) $(p=\mathrm{ns})$. The break down in surgery is shown in Table 1. Median length of stay for laparoscopic surgery was 10 days (range 6-15) compared with 14 days for open surgery (range 10-20) $(p=0.0001)$. Wound infection rate in laparoscopic surgery was $6 \%$ compared with $14.5 \%$ for open surgery. Positive margins occurred in nine patients $(3.4 \%)$ in the laparoscopic group compared with three $(3.9 \%)$ in the open group $(p=n s)$. The clinical anastomotic leak rate was $2.5 \%$ in the laparoscopic group compared with $4.5 \%$ in the open group.

Conclusion: Over $75 \%$ of colorectal cancers can undergo a laparoscopic resection with reduced hospital stay, decreased wound 
infection rate and equivalent oncological outcomes compared with open surgery. Laparoscopic surgery is now the standard of care for colorectal neoplasia in our institution.

Conflict of interest: None

Disclosures: None

Table 1

\begin{tabular}{lrrl}
\hline Surgery & Laparoscopic & $\begin{array}{c}\text { Open } \\
\text { Lap converted } \\
\text { to open }\end{array}$ \\
\hline Anterior Resection & 117 & 12 & 9 \\
$\begin{array}{l}\text { Abdominoperineal } \\
\quad \text { resection }\end{array}$ & 19 & 3 & 5 \\
Left hemicolectomy & 27 & 1 & 4 \\
Right hemicolectomy & 69 & 8 & 21 \\
Sigmoid colectomy & 27 & 2 & 4 \\
Subtotal colectomy & 4 & 2 & 3 \\
Hartmann's & 3 & 0 & 2 \\
Total & 266 & 28 & $n=342$ \\
\hline
\end{tabular}

\section{PROFILING BREAST CANCER IN OLDER PATIENTS. TIME TO INCREASE THE POPULATION SCREENING AGE?}

H.D. O'Connor, M.F. Cunningham, R. Salman, M.R. Kell, T.F. Gorey, M.A. Stokes

Department of Breast Surgery, Mater Misericordiae University Hospital, Dublin 7, Ireland

Background: Older patients make up to $40 \%$ of the breast cancer population. Currently screening includes women aged 50-64 years, despite international consensus to screen from 50 to 70 years. We profile the biological behaviour and treatment of breast cancer in older patients.

Methods: A prospective analysis of 328 patients aged 65-70 years with symptomatic breast cancer was performed. Biologic and clinical characteristics, local and systemic therapies, disease recurrence and overall survival were analysed. Comparisons were made with concurrently accumulated data from 266 women who presented with symptomatic breast cancer between 60 and 65 years of age.

Results: Breast cancers in patients aged $65-70$ years demonstrated similar hormonal profiles, tumour size variability and grade, as well as nodal and distal involvement in comparison to those in the 60-65 age group. Therapeutic surgical intervention, re-operation and adjuvant radiotherapy rates were comparable. Adjuvant chemotherapy was more prevalent in the younger cohort $(21$ vs. $52 \%, p<0.05)$, while increased hormonal therapy rates was observed in the older age group ( 66 vs. $38 \%, p<0.05$ ). In patients with lymph node-negative disease and/or tumours less than $2 \mathrm{~cm}$ who underwent therapeutic surgical intervention, 5-year survival rates were almost identical for both age groups (93 vs. $95 \%$ ), with age-adjusted mortality rate hazard ratios at $1.01(95 \%$ confidence interval $(\mathrm{CI})=0.98-1.04)$ for patients $65-70$ years old and $1.06(95 \% \mathrm{CI}=1.01-1.11)$ for patients 60 65 years old.
Conclusions: Tumour biology and overall breast cancer survival rates are comparable in symptomatic women aged 65-70 and 60-65 when detected early. The above data justifies treating $65-70$ year-olds similarly to $60-65$ year-olds.

Conflict of interest: None

Disclosures: None

\section{A DECADE OF BREAST CANCER SCREENING; ITS IMPACT OF PATIENT PRESENTATION TO A SYMPTOMATIC BREAST UNIT}

\author{
E. Molloy, M. Twyford, D. McCartan, Z. Al-Hilli, A.D.K. Hill \\ Department of Surgery, Beaumont Hospital, Dublin, Ireland
}

The establishment of BreastCheck aimed at early detection of breast cancer in women aged 50-64 years. Recent centralisation of cancer services has led to a significant increase in the number of patients diagnosed at designated symptomatic units. It is expected that this increase will only be reflected outside the screening age group. The objective of this study was to investigate the impact of the BreastCheck on the age distribution of patients newly diagnosed with breast cancer at Beaumont Hospital.

A retrospective study of breast cancer patients diagnosed between January 1996 and August 2009 was performed. Patient demographics and clinicopathological details were recorded. Patients were divided into three groups for analysis; the screening group 50-64, $<50$ and $>64$ years. Trends were analysed using linear regression.

A total of 1,262 patients were included with 390 in the screening age group. There was a significant decrease in cases seen in the screening age group as a percentage of the total number of cases over the study period $(p=0.002)$. A greater proportion of the caseload was due to patients in the 64 years and older age group $(p<0.001$ for regression) with no change in the percentage of tumours seen in the younger age group $(p=0.422)$. The mean tumour size did not significantly vary over the study period for any of the three groups examined.

The introduction of BreastCheck has led to a reduction in the number of women in the targeted age group presenting to a symptomatic breast unit.

Conflict of interest: None

Disclosures: None

\section{A PROSPECTIVE EVALUATION OF CARDIOVASCULAR RISK FACTORS-ARE WE TREATING TO TARGET?}

C. Mahon, Z. Martin, G.C. O’Brien, N. Cloete, M.P. Colgan, S.N. Haider, D.J. Moore, S.M. O’Neill, P. Madhavan

Department of Vascular and Endovascular Surgery, St James's Hospital, Dublin 8, Ireland

Patients undergoing vascular surgical procedures are a population known to be at high risk for peri-operative and late-cardiac events because of the high prevalence of underlying coronary artery disease. Effective reduction of the risk of cardiovascular disease through smoking cessation, correction of hyperlipidaemia and hypertension, 
and optimisation of diabetic control is essential in this cohort of patients to reduce peri-operative morbidity and mortality.

We prospectively collected data on 50 consecutive patients admitted to the vascular surgical service. This included both elective and emergency admissions. All patients had fasting cholesterol and lipids, HbA1C and four separate BP measurements at intervals performed to assess whether their identifiable risk factors were being treated to target. Target control was assessed using current European guidelines.

Thirty-six male and 14 female patients were included in the study. There were 14 patients with known diabetes. One patient was diagnosed with type 2 diabetes during his admission. Only five of these patients $(36 \%)$ had HbA1C levels within the recommended target range. $27 \%$ of patients had sub-optimal total cholesterol levels and $29 \%$ had sub-optimal LDL-C levels when tested. Only $65 \%$ of patients had their blood pressure adequately controlled according to recommended European guidelines. There were eight current smokers.

$70 \%$ of patients admitted had one or more risk factor that was not been treated to target. Greater emphasis must be placed on optimising treatment of identifiable cardiovascular risk factors, prior to patients being admitted to hospital, as treatment is currently sub-optimal. Conflict of interest: None

Disclosures: None

\section{CAN AUGMENTATION INDEX BE USED AS AN ACCURATE TOOL IN THE DIAGNOSIS OF PERIPHERAL OBSTRUCTIVE ARTERIAL DISEASE (POAD)? A CORRELATION STUDY}

\author{
G.P. Duff ${ }^{1}$, O. Galvin ${ }^{2}$, J. McCormack ${ }^{2}$, P.A. Grace ${ }^{1,2}$ \\ Graduate Medical School, University of Limerick ${ }^{1}$ Department of
} Vascular Surgery, Mid Western Regional Hospital, Limerick, Ireland ${ }^{2}$

Background: The gold standard for assessing POAD is the AnkleBrachial pressure index (ABPI) whereby a ratio is calculated between blood pressure measurements in the upper and lower limbs. A ratio of $<0.9$ is considered abnormal and indicative of POAD. Thus, the higher the APBI the more normal the circulation. Applanation tonometry is a novel method for assessing the compliance of the vascular tree. This modality measures the pulsatile expansion of the radial artery and uses a transfer function to calculate arterial stiffness expressed as the augmentation index (AI). Therefore, in healthy individuals with elastic arteries, the AI will be low or negative and high in patients with stiffer arteries from age or atherosclerosis.

Aims: The aim of this study was to evaluate the use of applanation tonometry in a clinical setting and to test the hypothesis that AI is a reliable indicator of a patient's vascular health. Factors affecting AI were also investigated.

Methods: We examined 60 patients in the Vascular Imaging Laboratory at the Mid-Western Regional Hospital Limerick between June and August of 2009. A range of inpatients and outpatients presenting to the laboratory were recruited to this study. Peers and colleagues also participated to act as healthy controls. APBI and AI were both measured in patients and controls, and relevant histories for risk factors were taken. APBI was measured using a Dynamap ${ }^{\mathrm{TM}}$ sphygmomanometer and AI with a Sphygmocor ${ }^{\mathrm{TM}}$ tonometer. Correlations between the two results were investigated. Data are presented as medians and ranges. Correlations were investigated using Spearman's rho and differences between groups were examined using the Kruskal-Wallis and Mann-Whitney $U$ tests.

Results: A strong negative correlation between APBI and AI was found (Spearman's rho $=-0.513, P<0.01$ ). The strongest affecting factor was found to be patient age, which had strong positive correlation with the patient's AI (Spearman's rho $=0.68, P<0.01$ ). Mann-Whitney testing showed there was significant increase in the AI scores of diabetic patients compared to normal patients (normal median 9 (-20-37) diabetic median $25(5-33), p<0.01)$ and in POAD patients compared to normal (POAD median $29(-1-52)$, $p<0.001)$. These data show a strong correlation between evidence of impaired peripheral circulation and increased arterial stiffness.

Conclusions: Augmentation Index is a reliable indicator of arterial stiffness and vascular health and its value increases with age. Further study into the clinical application of this quick and straightforward modality is warranted.

Conflict of interest: None

Disclosures: None

\section{RE-INTERVENTION FOLLOWING ENDOVASCULAR AORTIC ANEURYSM REPAIR: INCIDENCE IN A SINGLE CENTRE}

\author{
E.M. Quinn ${ }^{1}$, A.P. Coveney ${ }^{1}$, P.A. Cronin ${ }^{1}$, G. O'Brien ${ }^{1}$, G. Gosi ${ }^{1}$, \\ D. Tuite $^{2}$, G.J. Fulton ${ }^{1}$
}

Departments of Vascular Surgery ${ }^{1}$ and Radiology ${ }^{2}$, Cork University Hospital, Wilton, Cork, Ireland

Endovascular aneurysm repair (EVAR) is an increasingly used modality for the treatment of thoracic and abdominal aortic aneurysm [1]. Related complications, specifically those of endoleaks, have also assumed new importance in vascular surgical practice $[1,2]$. Endoleaks may require increased surveillance or further intervention.

The aim of this study was to investigate the re-intervention rate following EVAR. A retrospective review of all EVARs performed at a single institution over a 3 year period from January 2006 to November 2009 was performed and the incidence of development of endoleaks was recorded. Type of endoleak, surveillance and intervention, if performed, were reviewed.

Forty-six patients underwent EVAR, 44 abdominal EVAR and two thoracic EVAR. All were elective cases. Mean follow-up time was 22 months. Two patients died outside the postoperative period of unrelated causes. The others are enrolled in a follow up programme. Nine $(19 \%)$ patients developed endoleak; the majority of these were Type 2 endoleaks $(n=6)$. Four patients required intervention for endoleak; three for Type 1 endoleak and one for Type 2 endoleak. Three other patients required re-intervention for iliac limb thrombosis. Overall re-intervention rate after EVAR was $15 \%(n=7)$.

Endoleaks are a vascular complication encountered in the evolving era of EVAR. The majority of endoleaks are type 2 and resolve spontaneously, requiring only regular surveillance. Type 1 endoleaks require urgent re-intervention. New endovascular techniques are continually being developed to manage endoleaks and maintain EVAR durability. References:

(1) Van Marrewijk, Buth, Harris et al. (2002) Significance of endoleaks after endovascular repair of abdominal aortic aneurysms: The EUROSTAR experience. J Vasc Surg 35(3):461473

(2) Buth, Harris, Van Marrewijk et al. (2003) The significance and management of different types of endoleaks. Semin Vasc Surg 16(2):95-102

Conflict of Interest: None

Disclosures: None 


\section{CHOOSING TO BE A GENERAL SURGEON: A QUANTITATIVE STUDY OF IRISH BST TRAINEES}

\author{
S. McHugh ${ }^{1}$, M.A. Corrigan ${ }^{1}$, A. Sheikh ${ }^{1}$, W.A. Tanner ${ }^{2}$, \\ A.D.K. Hill \\ Department of Surgery, Beaumont Hospital, Dublin, Ireland ${ }^{1}$, Royal \\ College of Surgeons, St. Stephens Green, Dublin, Ireland ${ }^{2}$
}

Introduction: Irish general surgery (GS) faces a potential recruitment crisis with only 87 of 145 basic surgical training (BST) places filled in 2009, a trend mirrored in the UK and US. This is the first of a three part study to determine the factors which influence GS recruitment.

Methods: Candidates commencing BST training from 1978 to 2008 were identified through the RCSI database. Basic demographic details and examination results were recorded. Specifically all Irish trainees from 2002 to 2006 were followed up to determine their ultimate career choice, through examination of the medical council register, publications and internet.

Results: 2,536 candidates commenced surgical training during the study period of over 30 years. Of those whose nationality was traceable, 57\% were Irish. Between 2002 and 2006, 173 Irish candidates commenced BST. 114 were male $(66 \%)$. BST was completed by 152 trainees, with 135 obtaining MRCS exams and 46.7\% (63) passing in a single attempt. Career follow-up data was obtained on $127(73 \%)$. Seventy-five (59\%) candidates remained in surgery with $35(28 \%)$ choosing GS. Passing the MRCS examination in the first attempt was predictive of choosing GS compared to other surgical or medical subspecialties $(p=0.005)$. Gender and geographical location of training was not predictive of future career choices.

Conclusion: This study, the first part of a three part project, has demonstrated that only a quarter of Irish BST trainees choose GS. Those candidates that do so are likely to be more academically successful than their other surgical or medical colleagues, while gender or training location are not relevant factors.

Conflict of interest: None

Disclosure: None—project funded entirely by investigators

\section{SURGENT UNIVERSITY: THE ESTABLISHMENT AND EVALUATION OF A NATIONAL ONLINE CLINICAL TEACHING REPOSITORY FOR UNDERGRADUATE MEDICAL STUDENTS}

\author{
M.A. Corrigan ${ }^{1}$, A. Sheikh ${ }^{1}$, C.J. Shields ${ }^{2}$, H.P. Redmond ${ }^{3}$, \\ M.J. Kerin ${ }^{4}$, A.D.K. Hill ${ }^{1}$ \\ Department of Surgery, Beaumont University Hospital, Dublin, \\ Ireland ${ }^{I}$, Department of Surgery, Mater Misercordiae Hospital, \\ Dublin, Ireland ${ }^{2}$ Department of Surgery, Cork University Hospital, \\ Cork, Ireland ${ }^{3}$ Department of Surgery, Galway University Hospital, \\ Galway, Ireland ${ }^{4}$
}

Project aims: (1) Develop a new teaching strategy enhancing clinical teaching for medical students. (2.) Develop a national online repository system capable of delivering this teaching strategy. (3.) Evaluate the effectiveness of the system.

An online relational database was developed, facilitating students daily upload of clinical knowledge derived from ward based teaching in RCSI, NUI Galway and UCC (http://www.surgent.ie/ facts.php). These clinical facts were then peer reviewed by students for accuracy, and stored in a searchable online repository. Objective learning indicators were recorded over 8 weeks. Student evaluation was gathered via an online survey by the first group of 64 students.

Over the 8 week period the web-pages were accessed 54,061 times. A total of 6,105 facts were uploaded by 182 final medical students. Thirty-seven per cent $(2,269)$ of facts were consultant taught, with gastrointestinal surgery the most popular subject $(27 \%$ : 1,631). The systems search function was used 4,609 times. Of 64 students invited to evaluate the system, $32(50 \%)$ completed the online survey. They ranked their answers on a likert scale from 1 (worst) to 5 (best). Presented as a mean score, they ranked the following factors: ease of use; 4.7 , system beneficial; 4.0, retain system; 4.1. Eighty-eight per cent of students felt the system should continue for surgical attachments, while $78 \%$ felt it should be extended to nonsurgical attachments.

This study is the first to describe a coordinated national strategy to enhance clinical teaching. This system has been endorsed by students and represents an efficient integration of IT and traditional bedside teaching.

Conflict of interest: None-project funded entirely by investigators Disclosures: None

\section{MEDICAL STUDENT EDUCATION IN PRIVATE HEALTHCARE FACILITIES}

\author{
I. Robertson, G. Brady, A. Collins, D. O’Mara, C. Condron, \\ A.D.K. Hill \\ Department of Surgery, Beaumont Hospital, Royal College of \\ Surgeons in Ireland, Dublin 9, Ireland
}

Introduction: The use of private hospitals to facilitate the teaching of medical students is the norm in the United States and Australia where a combination of independently funded hospitals and publicly funded hospitals provide teaching facilities. Research has shown exposing students to private practice improves recruitment into specialities such as surgery and gives students more exposure to senior consultants. RCSI has completed the first year of a 1 week clinical attachment in Intermediate Cycle to the independent hospital sector which facilitated the exposure of our students to the changes in our healthcare landscape.

Aims: The aims of this study were to assess the benefits to medical students exposed to independent hospital clinical teaching.

Methods: During the intermediate cycle, 185 students were attached to various clinical services for 5 weeks, including 1 week at an independent hospital. Students were required to submit an electronic case up load with student reflection for each week in their e-portfolio. Students were asked to indicate which clinical competencies from our medical graduate profile (MGP) of 152 competencies that they carried out at each site either observed, simulated or under supervision.

Results: Students in independent hospitals performed as many 'performed supervised' competencies, and were involved in significantly more 'skill observed' competencies as their colleagues in teaching hospitals (See Table 1).

Conclusion: Rotations through independently funded hospitals provide opportunities which are difficult to obtain in teaching hospitals. Students in independent hospitals receive similar, if not better, exposure to many of the competencies examined when compared to traditional teaching hospitals. Private hospitals are a valuable instrument in medical undergraduate education.

Conflict of interest: None

Disclosures: None 
Table 1 MGP Competencies per student per rotation, ${ }^{*} p<0.05$, $t$ test

\begin{tabular}{lll}
\hline $\begin{array}{l}\text { MGP competencies } \\
\text { per student }\end{array}$ & $\begin{array}{l}\text { Independent } \\
\text { hospitals }\end{array}$ & $\begin{array}{l}\text { Teaching } \\
\text { hospitals }\end{array}$ \\
\hline Performed unsupervised & 1.9 & $6^{*}$ \\
Performed supervised & 9.95 & 10.57 \\
Skill observed & $15.42^{*}$ & 9.59 \\
Simulated & 0 & 5.78 \\
\hline
\end{tabular}

\section{SESSION 5: SURGICAL PRACTICE MANAGING CHANGE}

\section{CHANGING PATTERNS IN THE SURGICAL MANAGEMENT OF PARATHYROID DISEASE- A 10-Year Review}

\author{
T. McVeigh, A.J. Lowery, D. Dalton, C.A. Cowley, D. Quill, \\ M.J. Kerin \\ Department of Surgery, National University of Ireland, Galway, \\ Clinical Science Institute, Galway University Hospital
}

Parathyroid surgery has changed dramatically over the last decade, with a move to less invasive surgery, and increased reliance on preoperative and intra-operative localisation. We aimed to assess the changing practices and impact on the management of parathyroid disease at a tertiary referral centre.

Cohort analysis of all patients undergoing parathyroid surgery from 1998 to 2009.

Minimally invasive parathyroidectomy (MIP) was introduced in 2003.

Two hundred and thirty-four parathyroidectomies were performed, 181 following introduction of MIP, with an associated increase in mean number performed from 8 to 30 per year.

Preoperative Sestamibi in the MIP era correctly identified 87 adenomas, including two ectopic glands, as well as two carcinomas. However, a negative scan was also frequently associated with an adenoma.

Seventy-three patients undergoing MIP had intra-operative PTH monitoring. In 62 cases $(85 \%)$ PTH dropped by $>50 \% 5$ min post excision, and in five cases (7\%) PTH level halved $10 \mathrm{~min}$ post excision indicating successful identification of the diseased gland. There was no significant reduction in $\mathrm{PTH}$ in cases of multigland hyperplasia and one mediastinal parathyroid adenoma which required exploration.

The practice in our centre has shifted towards a less invasive approach to parathyroid surgery. Improved pre-operative localisation and utilisation of intra-operative PTH monitoring this has facilitated favourable changes in length of stay, operative times, extent of dissection, and number of patients treated.

Conflicts of Interest: None

Disclosures: None

\section{THE CHANGING FACE OF A VASCULAR SURGICAL UNIT; 1989-2008}

Z. Martin, G.C. O'Brien, N. Cloete, M.P. Colgan, S.N. Haider, D.J. Moore, S.M.O'Neill, P Madhavan

Department of Vascular and Endovascular Surgery, St James's Hospital, Dublin 8, Ireland

Since their introduction, endovascular techniques have firmly established their role in treating vascular patients and have dramatically changed the field of vascular surgery. In our unit all endovascular procedures are performed by vascular surgeons. To assess practice in our unit we have reviewed all procedures over a 20-year period

Data was collected prospectively over 20 years. We examined caseload volume under the headings, aorta (aneurysmal and occlusive disease), carotid, infrainguinal disease and amputation rate. For comparison, caseload was assessed in 5-year blocks. Venous disease was excluded.

The total number of arterial cases has increased $86 \%$ in the last 5 years compared with 15 years ago (1,599 vs. 861). Additionally $65 \%$ of all the arterial cases were managed using endovascular techniques compared with only $15 \% 15$ years ago. In the last 5 years $49 \%$ of aortic aneurysmal disease, $85 \%$ of aorto-iliac occlusive disease, $26 \%$ of carotid disease and $83 \%$ of infrainguinal disease was treated by endovascular techniques.

The greatest reduction in open cases was in aorto-iliac occlusive disease (70\% decrease) followed by infrainguinal disease (60\% decrease). The number of open aneurysm operations has not changed significantly (189 vs. 156 ) but $137 \%$ more aneurysms were treated in the last 5 years ( 370 vs. 156). The amputation rate $(n=100)$ remained constant.

These results show a significant increase in procedure volume over the last 20 years with the majority of arterial procedures now performed by endovascular techniques. If this experience is reflected nationally it emphasises the need for major changes in training vascular surgeons and in hospital infrastructure.

Conflict of interest: None

Disclosures: None

\section{THE IMPACT OF AN ANAESTHETIC PRE-OPERATIVE ASSESSMENT CLINIC ON LENGTH OF STAY IN VASCULAR SURGERY PATIENTS}

D.B. O'Connor, M. Cotter, O. Treacy ${ }^{2}$, D. O'Malley, A. McShane ${ }^{1}$, T. Owens, S.J. Sheehan, D. Mehigan, J. Dowdall, M.C. Barry

Departments of Vascular Surgery, Anaesthesia ${ }^{1}$ and Pre-operative Assessment Clinic ${ }^{2}$, St. Vincent's University Hospital, Elm Park, Dublin 4, Ireland

Major vascular surgery requires extensive medical and anaesthetic pre-operative work-up in elderly patients with multiple medical comorbidities. Pre-operative assessment clinics provide for out-patient anaesthetic and surgical assessment and reduce the risk of cancellation of procedures for medical reasons. The impact of such units on major vascular surgery has not been previously examined.

This pilot study examines the impact of a pre-operative assessment clinic on the vascular surgery service at St. Vincent's University Hospital and pre-operative lengths of stay. 
Patients undergoing abdominal aortic aneurysm repair, carotid endarterectomy and infra-inguinal bypass, referred to the pre-operative assessment clinic for elective vascular surgery between January and November 2009 were included. Ward consults and tertiary referral patients were excluded from analysis. Patients admitted for similar procedures during the year 2007 were used as controls.

For the 3 index procedures, 64 patients were assessed in the preoperative clinic. $44(68.8 \%)$ were deemed fit for surgery on initial assessment. A further 18 (28.1\%) were declared fit following further medical consultation and $2(3.1 \%)$ were deemed unfit. Mean preoperative LOS in this group was 1.974 days (median $=1$ ) compared to 5.45 (median $=4)$ in the control group $(p<0.0001$, Mann-Whitney $U$ test). Of the 38 patients who have undergone surgery to date, 25 were admitted on the day before surgery compared to only 12 of 64 patients in the control group. $(p<0.0001$, chi-square test).

A pre-operative assessment clinic reduces the risk of cancellation of major elective procedures for medical reasons and significantly shortens pre-operative length of stay and costs.

Conflict of interest: None

Disclosures: None

\section{IS LASER BETTER? A PROSPECTIVE OUTCOME COMPARISON OF VARICOSE VEIN THERAPIES}

\section{D.C. O’Neill, S.B. O’Neill, A.P. Coveney, D.F. O’Brien, G. Fulton}

Department of Vascular Surgery, Cork University Hospital, Cork, Ireland

The gold standard treatment of varicose veins is high ligation and stripping (HL\&S). Endovenous laser therapy (EVLT), a minimally invasive ultrasound guided treatment using laser energy offers an alternative.

The aim of this study was to compare patient outcome following both varicose vein therapies used in Cork University Hospital, by comparing the immediate quality of life (QoL) outcomes following EVLT and HL\&S, by assessing pain management post-operatively and by comparing initial patient satisfaction.

Consenting consecutive patients undergoing both varicose vein treatments were recruited over a 4 month period. QoL outcomes of the participants were assessed using the Aberdeen Varicose Vein Questionnaire (AVVQ) pre-operatively and at 10 days and 6 weeks post-operatively. Participants also completed two separate evaluation questionnaires at 10 days and 6 weeks post treatment, respectively.

Twenty-one patients underwent EVLT and 26 underwent HL\&S. Baseline median AVVQ scores were 16.34 (IQR 9.78-21.09) for EVLT and 16.74 (IQR 10.83-22.21) for HL\&S. Follow-up at 6 weeks was $95.2 \%$ in the EVLT group and $92.3 \%$ in HL\&S group. At 6 weeks, median AVVQ scores were 5.59 and 4.99, respectively, showing no significant difference $(p=0.268)$ in improvement between the groups. There were no significant difference in pain level at $48 \mathrm{~h}$ post-operatively ( $p=0.746$ ) or number of days taking pain relief $(p=0.772)$. Satisfaction levels with the overall procedure at 6 weeks were $95 \%$ for EVLT and $91.7 \%$ for HL\&S.

EVLT is well tolerated and provides similar improvements in QoL outcomes, and post-operative pain and satisfaction levels, to that of HL\&S.

Conflict of interest: None

Disclosures: None

\section{SURGICAL ASSESSMENT UNIT-AN EFFECTIVE STRATEGY FOR STREAMLINING ACUTE SURGICAL ADMISSIONS}

\author{
A. Loftus, P. Finnegan, J. Smith, P. Burke \\ Department of Surgery, Mid-Western Regional Hospital, Limerick, \\ Ireland
}

Introduction: In October 2009 a dedicated surgical assessment unit (SAU) was set up in the Mid Western Regional Hospital (MWRHL) to optimize the initial assessment of acute adult surgical patients. This coincided with the centralization of all acute surgical services in the MWRHL. A prospective audit was performed on patients admitted to the surgical assessment unit during its first 2 months of operation. We examined the number of admissions, referral source, time to bed, time to theatre and inappropriate referrals. We then compared the time to bed and time to theatre with acute surgical emergencies through the $\mathrm{A} / \mathrm{E}$ department for the same time period.

Results: In total there were 255 referrals to the SAU, 150 (59\%) from GP's and 105 (41\%) from satellite Local Emergency Centers. 60\% of patients referred required admission with $10 \%$ of referrals considered inappropriate. $77 \%$ of patients admitted through the SAU received a bed within $3 \mathrm{~h}$ as compared to $1.5 \%$ through A/E, while only $6 \%$ of SAU patients waited longer then $6 \mathrm{~h}$ compared to $72 \%$ of patients admitted through A/E. $18 \%$ of SAU patients requiring emergency surgery had their operation within $5 \mathrm{~h}$ compared to $7 \%$ that attended to $\mathrm{A} / \mathrm{E}$.

\begin{tabular}{lll}
\hline & SAU & A/E \\
\hline $\begin{array}{l}\text { No. of Admissions } \\
\text { Time to Bed }\end{array}$ & 153 & 205 \\
$0-3 \mathrm{~h}$ & 117 & 3 \\
$3-6 \mathrm{~h}$ & 27 & 54 \\
$>6 \mathrm{~h}$ & 9 & 148 \\
Time to theatre & & \\
$0-5 \mathrm{~h}$ & 2 & 2 \\
$5-24 \mathrm{~h}$ & 3 & 21 \\
$>24 \mathrm{~h}$ & 6 & 4 \\
\hline
\end{tabular}

Conclusion: The establishment of an Acute Surgical Assessment Unit facilitates speedier assessment of patients with prompter decision making and subsequent definitive management. This improves patient care with a potential reduction of inpatient bed days along with reduced pressure on $\mathrm{A} / \mathrm{E}$ facilities.

Conflict of interest: None

Disclosures: None

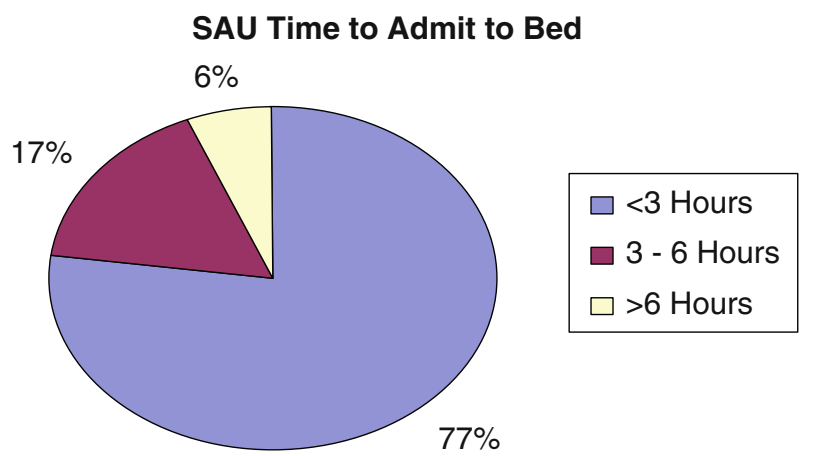




\section{IMPLEMENTATION OF RECONFIGURATION OF ACUTE SURGICAL SERVICES IN THE MID- WESTERN REGION}

\author{
J. Moloney, F.M. Smith, E. T. Condon, D. Waldron, P. Burke \\ Department of Surgery, Mid-Western Regional Hospital, Limerick, \\ Ireland
}

Introduction: Acute surgical services have recently been reconfigured. Emergency and trauma services have been transferred from three peripheral hospitals to the Mid-Western Regional Hospital. In addition, a surgical assessment unit (SAU) has been established, which bypasses $\mathrm{A} \& \mathrm{E}$, in an attempt to expedite review and formulation of a management plan. This study provides an overview of service provision and early data on the implementation of reconfiguration.

Methods: The number of general surgical admissions, transfers and emergency operations performed in MWRH before and after reconfiguration were assessed. Early data pertaining to the establishment of the SAU were obtained and analysed.

Results: Since reconfiguration there has been an overall increase in the number of surgical admissions by $23 \%$ during weekdays and $43.5 \%$ at weekends. The weekly number of all emergency cases performed has increased by $38 \%$ since reconfiguration started. General surgical cases have increased by $41 \%$ from an average of 76 per month in April 09 to 107 per month in October 09. In a 2 week period (01/10-14/10), the SAU has seen an intake of 70 patients, with arrival to decision in less than $2 \mathrm{~h}$ occurring in $\sim 56 \%$.

Conclusions: Since the introduction of reconfiguration there has been an overall increase in emergency general surgical workload of $>40 \%$. The increases in numbers of surgical patients needing to be assessed have necessitated changes in the working patterns and number of staff on call. This includes the introduction of the SAU, which has decreased the number of patients needing to be seen in A\&E. Conflict of Interest: None

Disclosures: None

\section{RAPID ACCESS COLORECTAL CLINIC- 5 YEAR OUTCOMES}

\author{
A. Ahmed, E. Meyers, W.O. Kirwan \\ Department of Academic Surgery, Cork University Hospital, Cork, \\ Ireland
}

Increasing pressures on surgical outpatient clinics have caused unacceptable waiting times for patients with high risk colorectal symptoms. Rapid access colorectal clinics (RACC) provide flexible sigmoidoscopy within 2 weeks for these patients after appropriate GP referral and barium enema if needed. An established RACC reported a $7.1 \%$ detection rate for colorectal cancers (CRC) 1. Our RACC service was initiated in Cork University Hospital in June 2001.

The aims of the study are to evaluate the referral-review duration, symptoms, clinical findings, investigations (sigmoidoscopy and barium enema), histology and the cost effectiveness of day ward colonoscopies with sedation versus sigmoidoscopy with barium enema.

This retrospective study is based on patients clinical notes collected between 2001 and 2006. It has included 450 patients referred to RACC, of which $50.6 \%$ are males and $49.4 \%$ females with average ages of 51.6 and 54.6 years, respectively. The mean duration between GP referral and outpatients visits was 19.2 days. The main indications for referral were rectal bleeding (55\%), change in bowel habit $(52 \%)$, and abdominal pain $(18 \%)$. The average distance of the sigmoidoscope was $53.9 \mathrm{~cm}$ (standard error 1.39) for males and $48.7 \mathrm{~cm}$ (standard error 1.79) in females. CRC detection rate was $7.9 \%$. $17 \%$ of all referrals had haemorrhoids, $12 \%$ had diverticular disease and $31 \%$ of patients showed no pathology in their investigations.

The results of this study are similar to previously published data and demonstrate that RACC clinics would reduce the outpatient waiting times and free beds routinely used for colonoscopies, hence increasing cost effectiveness.

\section{Reference:}

Lamah M, Ahmad SM, Charalampopoulos A, Ho J, Leicester RJ (2000) Three-year evaluation of a rapid-access coloproctology clinic. Dig Surg 17:150-153

Conflict of interest: None

Disclosures: Approved

\section{THE IMPACT OF CASELOAD ON PRACTICE PATTERNS IN BREAST CANCER; EVIDENCE FROM THE UK BREAST SCREENING PROGRAMME}

\author{
N. Marshall ${ }^{1}$, R.W. Glynn ${ }^{1}$, O. Kearins ${ }^{2}$, G. Lawrence ${ }^{2}$, M.J. Kerin ${ }^{1}$ \\ Department of Surgery, National University of Ireland Galway, \\ Ireland $^{l}$ West Midlands Cancer Intelligence Unit, NHS Breast \\ Screening Programme, Birmingham, $U K^{2}$
}

Studies examining the relationship between caseload and surgical practice in breast cancer have generally involved a relatively small number of units or patients, and have been based on practice patterns in the 1990s. We aimed to examine the caseload volume of screening units and individual breast cancer surgeons across the UK, and to investigate the relationship between these volumes and practice patterns in the contemporary setting.

The non-operative and operative history of screen-detected breast cancers, diagnosed in women who were invited for screening between 2000 and 2008 within the UK Breast Screening Programme, was extracted from national databases. This information was then correlated with unit- and individual surgeon caseload.

There were 13,782,055 screening events over the study period. Unit size did not affect the cancer detection rate $(p=0.786)$. The number of surgeons responsible for less than 10 cases annually decreased from 32.9 to $23.5 \%$; this $23.5 \%$ managed just $2.4 \%$ of the 16,676 patients diagnosed through the programme in 2007/2008. The median rate of mastectomy was $27.8 \%$ (IQR 21.35-36.13) whilst that for immediate reconstruction was $7.01 \%$ (IQR 0-17.31). The median rate of SNB in invasive cancers was 2.2\% (IQR 0-25.57). Higher surgeon caseload was associated with decreased mastectomy rates $(p=0.000)$, and increased use of immediate reconstruction $(p=0.000)$ and SNB $(p=0.000)$.

Whilst many surgeons are still practicing out-with BASO guidelines, significant improvements have been made. The important variable in terms of practice patterns is the number of cancers each individual surgeon deals with, and the caseload of individual units is less important.

Conflict of interest: None

Disclosures: None 


\section{DETRIMENTAL IMPACT OF THE EUROPEAN WORKING TIME DIRECTIVE (EWTD) ON SENIOR HOUSE OFFICER OPERATIVE EXPERIENCE}

\author{
K.J. Breen, A.M. Hogan, E. O'Halloran, K. Mealy \\ Department of Surgery, Wexford General Hospital, Wexford, Ireland
}

Concerns have been voiced regarding the impact of EWTD on surgical training. Following its introduction (August 2009) in Wexford General Hospital surgical house officers (SHOs) are required to leave the hospital at 10am the morning after call. This study investigates the consequences of this practice on operative experience gained by six SHOs in comparison to their predecessors. A prospectively maintained database of surgical procedures was interrogated. Operative experience of surgical SHOs over a 3 month period (August-October 2009) was compared with that of colleagues at the same level 1 year earlier. The primary endpoint was overall operative exposure of SHOs. Subgroup analysis was preformed of cases (performed as operating surgeon and as first assistant for major and minor procedures and by consultant). Comparison was made of operative exposure of SHOs on pre- and post-EWTD rotas. SHOs operating within the constraints of EWTD were involved in a total of 211 operations. This was compared to 289 for SHOs in the same period of 2008, a decrease of $27 \%$. PreEWTD SHOs had been 1st surgeon in a total of 61 cases (35 minor, 26 major) compared to 42 (26 minor, 16 major) in the post EWTD group, demonstrating a reduction of $31 \%$. The present data set demonstrates a significant reduction in operative experience gained by surgical SHOs as a direct result of the implementation of the EWTD.

Conflict of interest: None

Disclosures: None

\section{PREVENTABLE DELAYS IN THE ELECTIVE SURGICAL PATIENT IN AMNCH, TALLAGHT. A RETROSPECTIVE STUDY}

\author{
K. O’Sullivan, C. Mc Donald, P.N. Neary, E. Eguare \\ Department of Surgery, AMNCH, Tallaght
}

Elective case delays are upsetting to patients and a major drain on healthcare resources. We investigated the impact such delays have had on the colorectal service in $\mathrm{AMNCH}$, Tallaght. A retrospective review of admissions for elective bowel resection was performed over a 6 month period. A total of 90 patients were included; 47 male, 43 female. We found that a total of 54 patients or $60 \%$ suffered preoperative delays.

These delays were examined and divided into preventable and unpreventable groups. Preventable reasons included were

1. Preoperative cancer staging

2. Delays on medical grounds requiring pre-operative assessment to ascertain anaesthetic safety
We found that preventable delays totalled 44 patients or $81 \%$ of delayed cases. Of these, 38 or $86.36 \%$ were due to staging procedures for cancer and the total bed occupancy amounted to 120 days. We also found that 6 or $13.63 \%$ of these patients were delayed preoperatively due to purely medical reasons. This group spent a total of 36 days in hospital undergoing medical assessment. This could have been organised in the outpatient setting such as cardiology $n=6$, ECHO $n=3$, angiogram $n=1$, respiratory $n=2$, vascular $n=1$. Combined cost of preventable bed occupancy days totalled $€ 107,484$

Pre-operative assessment clinics have been proven to reduce elective case delays on medical grounds and could be expanded to facilitate staging and work-up. This study suggests that with upgrade and expansion of this pre-existing facility, significant improvements could be made to both the efficiency and cost-effectiveness of the service.

Conflict of interest: None

Disclosures: None

\section{TRENDS AND ATTITUDES TO LAPAROSCOPIC APPENDECTOMY IN IRELAND}

\author{
D.P. McCartan, F.J. Fleming ${ }^{1}$, A.D.K. Hill
}

Department of Surgery, Beaumont Hospital, Dublin 9, Ireland, Department of Surgery, University of Rochester Medical Centre, Rochester, New York, USA ${ }^{l}$

The role of laparoscopy in the surgical management of appendicitis has yet to be definitively established. The objective of this study was to examine Irish trends in the management of acute appendicitis and to assess current opinion among Irish surgeons towards LA.

Using ICD codes, data was obtained from the Irish Hospital in Patient Enquiry system for all adult patients discharged with a diagnosis of appendicitis between 1999 and 2007 accounting for 38,659 appendectomies. An anonymous postal survey was sent to all general surgeons of consultant and registrar level in Ireland to assess current attitudes to the use of laparoscopic appendectomy.

The use of LA increased throughout the study period and in 2007 was the most common approach for appendectomy. Multivariate analysis revealed younger age, female sex and residence in higher density population areas as predictive factors for undergoing LA. While $97 \%$ of surgeons reported current use of laparoscopy in patients with acute RIF pain, in most cases the use was selective with only $40 \%$ using laparoscopy in all patients. Surgeons in university teaching hospitals were more likely to report using a laparoscopic approach for all cases than those in regional or general hospitals but no difference existed between subspecialty or for number of years in practice.

While the rate of LA has substantially increased in Ireland over the study period, differences in the perception of benefit of the laparoscopic approach amongst surgeons appears to be an important factor in determining the operative approach in the management of appendicitis.

Conflict of interest: None

Disclosures: None 


\section{INITIAL ENCOUNTER WITH THORACIC ENDOVASCULAR ANEURYSM REPAIR (TEVAR) FOR THORACO-ABDOMINAL PATHOLOGY (TAP) IN A TERTIARY REFERRAL VASCULAR CENTRE. A TECHNICAL CHALLENGE OF DEBRANCHING, CHIMNEYS, SNORKEL ENDOVASCULAR GRAFTS IN THE ERA OF HYBRID SURGERY?}

\author{
P. Waters, G. Mann, W. Tawfick, M. Tubassam, S. Sultan \\ Department of Vascular Surgery, UCHG
}

Management of TAP is dependent on anatomical configuration that is often obscured by the involvement of the visceral arteries, especially Thoraco-abdominal aneurysms.

We convey our early experience in management of TAP. Primary endpoints were aneurysm free survival and 30 days aneurysm related mortality. Secondary endpoints were freedom from MACE, technical success rate measured by Graft migration, aneurysm expansion, conversion to open repair or graft infection.

Twenty-one TEVAR procedures were performed for 19 patients (10 females: 9 Males) over a period of 2 years of which two were emergencies. 13 were for thoracic aneurysms type three, 5 thoracoabdominal aneurysms type four, 1 aortic dissection Type B, 1 adult aortic coarctation and 1 spontaneous aortic rupture.

Six patients underwent a one/two stage hybrid debranching of visceral or carotid vessels followed by TEVAR. 4 patients underwent chimney or Snorkel Endografting of Subclavian or renal vessel s. Three patients required Endopaving and cobbling. No patients developed aneurysm rupture, paraplegia or stroke.

No cases of endoleak or aneurysm expansion were witnessed. Two patients experienced distal graft migration and required re-intervention. 30 day morbidity was 1 acute tubular necrosis and 1 lower respiratory tract infection. There was two mortalities within 30 days for the two emergencies of which one was a HIV patient with syphilis.

TEVAR with debranching, chimney or snorkel grafting of visceral vessels is safe, prudent and economically viable. TEVAR bestows minimally invasive unorthodox techniques to open TAP repair in challenging TAP anatomies with less morbidity and mortality. Conflict of interest: None

Disclosures: None

\section{SESSION 6: ORTHOPAEDIC SESSION}

\section{THE EFFECTS OF RESISTANCE TRAINING AND NEUROMUSCULAR ELECTRICAL STIMULATION IN KNEE OSTEOARTHRITIS- A RANDOMIZED CONTROL TRIAL}

\author{
R. Bruce-Brand ${ }^{1}$, J.M.O'Byrne ${ }^{1}$, N. Moyna ${ }^{2}$ \\ Department of Trauma and Orthopaedics, Cappagh National \\ Orthopaedic Hospital, Finglas, Dublin 11, Ireland ${ }^{1}$, Department of \\ Human Health and Performance, Dublin City University, Dublin, \\ Ireland $^{2}$
}

Background: Knee osteoarthritis is responsible for more chronic disability in elderly people than any other medical condition. Quadriceps femoris muscle weakness has long been associated with disuse atrophy in symptomatic knee osteoarthritis but more recently implicated in the aetiology of this condition. The purpose of this study was to assess the benefits of two interventions aimed at increasing quadriceps strength in subjects with moderate to severe knee osteoarthritis.

Methods: Twenty-eight patients, aged 55-75 years, diagnosed with moderate to severe knee osteoarthritis were recruited and randomised to either a 6-week home resistance-training exercise program or a 6-week home neuromuscular electrical stimulation (NMES) program. An additional eleven patients matched for age, gender and osteoarthritis severity formed a control group, receiving standard care. The resistance-training group performed six exercises three times per week, while the NMES group used the garment stimulator at the maximum intensity tolerated for $20 \mathrm{~min}$ five times per week. Outcome measures included isometric and isokinetic quadriceps strength, functional capacity ( $25 \mathrm{~m}$ walk test, chair rise test, stair climb test), Western Ontario and McMaster Osteoarthritis Index (WOMAC) and Short Form 36 (SF-36) health surveys. These measures were assessed at baseline, pre-intervention (after familiarisation), post-intervention and at 6-weeks post-intervention. Additionally, quadriceps cross-sectional area (via MRI) and muscle atrophy/hypertrophy gene expression (via vastus lateralis biopsy) were assessed pre- and post-intervention.

Results: Both intervention groups showed significant improvements in all functional tests (e.g. in the stair test, a $22 \%$ improvement in the exercise group versus $17 \%$ for the NMES group), in the SF36 health survey ( 25 and $22 \%$ respectively), and in quadriceps cross-sectional area $(4.3$ and $5.4 \%)$ immediately post-intervention. An increase in isokinetic strength was seen in the exercise group only (11\%). WOMAC score improved only for the NMES group (19\%). With the exception of isokinetic strength, all benefits were maintained 6 weeks post-intervention.

Conclusions: Both a 6-week home resistance-training program and a 6-week home NMES program produced significant improvements in functional performance as well as physical and mental health for patients with moderate to severe knee osteoarthritis. Home-based NMES is an acceptable alternative to physical therapy for patients with knee osteoarthritis, and is especially appropriate for patients who have difficulty complying with an exercise program.

Conflict of interest: None

Disclosures: None

\section{EFFECT OF VIBRATION ON THE SHEAR STRENGTH OF IMPACTED BONE IN REVISION HIP SURGERY}

\author{
S. Brennan ${ }^{1,2}$, K. Ryan ${ }^{1}$, D. Brabazon ${ }^{1}$, J. O’Byrne ${ }^{2}$ \\ Department of Orthopaedic Surgery, Cappagh Hospital, Dublin, \\ Ireland $^{l}$, Department of Mechanical Engineering, Dublin City \\ University, Dublin, Ireland
}

Studies on soil mechanics have established that when vibration is applied to an aggregate, it results in more efficient alignment of particles and reduces the energy required to impact the aggregate. Our aim was to develop a method of applying vibration to the bone impaction process and assess its effect on the mechanical properties of the impacted graft.

Phase 1. Eighty bovine femoral heads were milled using the Noviomagus bone mill. The graft was then washed using a pulsed lavage normal saline system over a sieve tower. A vibration impaction device was developed which housed two 15V DC motors with eccentric weights attached inside a metal cylinder. A weight was dropped onto this from a set height 72 times so as to replicate the bone impaction process. A range of frequencies of vibration were tested, as measured using an accelerometer housed in the vibration chamber. 
Each shear test was then repeated at four different normal loads so as to generate a family of stress-strain curves. The Mohr-Coulomb failure envelope from which the shear strength and interlocking values are derived was plotted for each test.

Phase 2. Experiments were repeated with the addition of blood so as to replicate a saturated environment as is encountered during operative conditions.

Relatively dry graft impacted with the addition of vibration showed improved shear strength at all frequencies of vibration when compared to impaction without vibration. In our system the optimal frequency of vibration was $60 \mathrm{~Hz}$. Under saturated conditions the addition of vibration is detrimental the shear strength of the aggregate. This is secondary to decreased interlocking between particles and may be explained by the process of liquefaction.

Conflict of interest: None

Disclosures: None

\section{HIP FRACTURE IN THE ELDERLY: AN INDICATOR OF HEALTHY LIFE YEARS}

\section{E. Keane, C. Green, C. Fitzpatrick, K. O’Rourke}

Department of Trauma and Orthopaedics, St. Vincent's University Hospital, Dublin 4, Ireland

As a result of an increase in the elderly population the number of hip fractures was expected to rise dramatically and put significant strain on the provision of medical care. However, this rapid rise in fracture rate has not been universally observed. Healthy Life Years (HLY) is a measure that reflects the fact that not all life years are spent in perfect health with disability becoming more common in the elderly. Hip fractures in the elderly are an important source of morbidity and mortality. Healthy Life Years is used to monitor health as an economic factor with a strong relationship between gross domestic product (GDP) and healthy life years demonstrated in a number of studies. The purpose of this study was to examine the incidence of hip fractures in over time in our centre relative to the population of the hospitals catchment area and to determine if a hip fracture could be used as a useful marker of health life years.

All hip fractures from the unit over 20 years were identified. Population data for those over 65 in the catchment area of our hospital was acquired. The rate of fractures occurring each year relative to the population was determined. The results were split into age groups. There was a strong correlation between the population rise and number of fractures $(p=0.77)$. But there was no significant difference in the rate of fracture over time $(p=0.41)$. However, the average age at which fracture occurred increased by 2 years over the period of the study. In addition we show the overall trend in the rate of fractures to decrease in the younger age groups and increase in the older age groups.

Our study illustrates that although the number of hip fractures in the over 65 age group is rising as the elderly population expands it is not doing so at the dramatic rate predicted. This seems to be because of the shift in the fracture pattern with a relative decrease in the younger patients and a relative increase in the extreme elderly. This represents an increase in the number of healthy life years for the population using hip fracture as a marker. These figures are in keeping with the latest national figures for health life years using other measurements. This study suggests that due to the continued economic growth in Ireland over the last 80 years the number of healthy life years has increased and therefore although the population has increased we have not seen the envisaged surge in hip fractures. Furthermore we suggest that the age at which hip fracture occurs can be used as a useful determinant of healthy life years. Conflict of interest: None

Disclosures: None

\section{BIOMECHANICAL COMPARISON OF THE DYNAMIC HIP SCREW (DHS), THE X-BOLT AND THE DHS BLADE}

\author{
F. O’Neill ${ }^{1}$, T. McGloughlin, F. Condon, M. Walsh
}

Centre for Applied Biomedical Engineering Research (CABER), Department of Mechanical and Aeronautical Engineering and Materials and Surface Science Institute (MSSI), University of Limerick, Midwestern Regional Orthopaedic Hospital Croom/MidWestern Regional Hospital, Dooradoyle, Limerick, Ireland

Hip fractures are one of the most common fractures in the elderly population. Hip fractures can be either intracapsular or extracapsular such as intertrochanteric fractures and for the purpose of this study, the focus on the treatment of intertrochanteric fractures. The dynamic hip screw (DHS) was initially introduced by Clawson (1964) and has been widely adopted as the implant of choice in the treatment of these fract ures. Its most common mode of failure is the so called cut out of the lag screw from the femoral head.

There have been attempts to redesign the lag screw element of the implant to help gain better anchorage within the bone and some of the latest attempts at this are the $\mathrm{x}$-bolt and the DHS blade.

The objective of this study was to biomechanically test and compare the dynamic hip screw with the $\mathrm{x}$-bolt and the DHS blade to help determine which device provides the better anchorage within the bone. Subsidence (pushout), pullout and the torsional stability of each device in synthetic bone blocks of different densities was tested. Then subsidence (pushout) in osteoporotic cadaveric femoral heads was tested. The cadaveric femoral heads were dexa scanned prior to testing to determine bone mineral density and femoral heads of similar densities were evenly distributed between the devices for testing.

Results presented compare these biomechanical forces $f$ or each of DHS, x-bolt and DHS blade devices.

Conflict of Interest:

There is no conflict of interest as this has been a self financing project without any outside funding.

Disclosures:

Sota Orthopaedics provided the x-bolt samples, DHS screws and surgical instruments free of charge. Fanning Healthcare provided DHS blades, DHS screws and surgical instruments free of charge.

\section{HIP AND MUSCLE FORCES IN HIP REPLACEMENT SUBJECTS}

\author{
D. O'Reilly ${ }^{1}$, O. Flannery ${ }^{2}$, M. Donoghue ${ }^{1}$, F. Condon ${ }^{2}$, M. Walsh ${ }^{1}$ \\ Centre for Applied Biomedical Engineering Research, Department of \\ Mechanical and Aeronautical Engineering, University of Limerick, \\ Limerick, Ireland ${ }^{l}$, St. Nessan's, Mid-Western Regional Hospital, \\ Croom, Limerick, Ireland ${ }^{2}$
}

The aim of this study was to find the forces at the hip joint and the forces of the surrounding muscles for subjects after a total hip replacement. Subjects with a variety of head sizes and a variety of surgical approaches were also examined. This was done by using motion analysis and LifeMod, a biomechanics modelling software.

Motion analysis was carried out using 6 inferred cameras and 15 markers placed on the subject. The subjects were asked to carry out several everyday activities such as walking, sitting and standing. The data was recorded and processed using EVaRT 5.0 (Santa Rosa, CA, USA). The marker positions were then used as $t$ he motion input for models created in LifeMod (San Clemente, CA, USA). From these 
biomechanical models results can be calculated for all hip and muscle forces. Forces were found at all joints and muscles in the lower limbs for all the everyday activities tested. The average peak force for walking was $218 \%$ of body weight. This is comparable to Bergmann et all who found peak forces of $238 \%$ and Brand et al 2 with forces of $250 \%$

The results found demonstrate that biomechanics modelling software such as LifeMod can be used to find both muscle and joint forces for everyday activities. Comparisons have been drawn between the posterior and anterolateral surgical approaches. The difference in hip forces between subjects with large stem heads were also compared to subjects with a normal sized head.

(1) Bergmann G, Deuretzbacher G, Heller M, Graichen F, Rohlmann A, Strauss J, Duda GN (2001) Hip contact forces and gait patterns from routine activities. J Biomech 34:859-871

(2) Brand RA, Pedersen DR, Davy DT, Kotzar GM, Heiple KG, Goldberg VM (1994) Comparison of hip force calculations and measurements in the same patient. J Arthroplast 9(1):45-51

Conflict of interest: None

Disclosure: None

\section{THE IRISH HIP FRACTURE DATABASE: 1 YEAR RESULTS FROM A REGIONAL HOSPITAL IN MID-WEST IRELAND}

\author{
A. Daly ${ }^{1}$, T. Burke ${ }^{2}$, D. O’Farrell ${ }^{2}$, L. D'Souza ${ }^{2}$, E. Masterson ${ }^{2}$, \\ F. Condon ${ }^{2}$
}

Orthopaedic Clinical Nurse Specialist, Mid-Western Regional Hospital Limerick, Ireland ${ }^{I}$, Consultant Orthopaedic Surgeons, MidWestern Regional Hospital Limerick, Department of Orthopaedics, Mid-Western Regional Hospital, Limerick, Ireland ${ }^{2}$

In 2007, 11 orthopaedic sites involved in hip fracture care were invited to participate in the Irish Hip Fracture Database (IHFD), a web based data capture RioMed ${ }^{\mathrm{TM}}$ system, sponsored by Merck Sharp and Dho$\mathrm{me}^{\mathrm{TM}}$. This was to facilitate in prospectively reviewing a consecutive series of hip fracture patients' journey through the health care system. To assist in the examination of current standards of care and benchmark with other trauma orthopaedic departments in Ireland and abroad.

A standard core dataset was pre established by the IHRD. Data was collected during the acute stay and follow up data was collected at 30 and 120 days post-op in the out-patients department or by telephone. Case mix variables included age, gender, fracture type, pre fracture residence, pre fracture mobility and the Society of Anaesthesiologists (ASA) score.

Management variables included time from fracture to admission and to operation, reasons for delay, types of surgeries, length of hospital stay and destination on discharge.

30 and 120 day follow up assessment allowed for comparison of mobility, residential status, re-operation and mortality rates.

293 patients were included in the IHFD; patients who were treated conservatively were excluded. $75 \%$ were females and $25 \%$ males. Fracture type and surgeries were classified. The majority had an ASA score of $3(54 \%)$. The average wait in A $+\mathrm{E}$ for admission was $5 \mathrm{~h}$ $53 \mathrm{~min}$ and $30 \%$ waited greater than $48 \mathrm{~h}$ for surgery, the main reason for delay being administrative or logistical. The average length of hospital stay was 11 days, this less than the national average of 18 days. Post operative mobility and use of waking aids were established and compared. An increase of $22 \%$ of residence in nursing homes at 120 days and a mortality rate of $14 \%$ at 120 days was revealed. All complications that required re-admission or further surgery were recorded. Results are compared with care standards developed by the British Orthopaedic Association (Care of people with fragility fractures, 2007) and Scottish Guideline SIGN No:111. Conflict of interest: None

Disclosures: None

\section{FEMORAL NECK NARROWING AND RESURFACING ARTHROPLASTY: AN EARLY OR LATE OSTEOLYTIC PHENOMENON?}

\author{
E. Cullen ${ }^{1}$, A. Simpkin ${ }^{2}$, D. Bergin ${ }^{3}$, K. Kaar ${ }^{1}$, W. Curtin ${ }^{1}$ \\ Department of Orthopaedics, Merlin Park Hospital, Galway, \\ Ireland $^{l}$, Health Research Board, Clinical Research Facility, NUI, \\ Galway, Ireland ${ }^{2}$ Department of Radiology, Merlin Park Hospital, \\ Galway, Ireland ${ }^{3}$
}

Resurfacing hip arthroplasty has had several modifications in component design and tribology since Sir John Charnley's Teflon components of the 1950's. There is much work published on the advantages over total arthroplasty surgery, especially in a younger patient. However, reports show narrowing at the bone implant interface as a recognised phenomenon. Our aim is to determine the implication/s of this phenomenon and whether it occurs early or late. This retrospective cohort study looked at patients undergoing metal on metal resurfacing arthroplasty over a 3 year period by two surgeons at a tertiary referral centre. Components implanted were either an Articulating Surface Replacement (ASR, DePuy International) or a Birmingham Hip Resurfacing (BHR, Smith and Nephew) system. We calculated femoral neck narrowing in pelvic radiographs in the anteroposterior plain using a unique conversion coefficient derived from the mouth diameter of the prosthesis. Several other parameters were measured for correlation and significance to femoral neck narrowing using multivariate regression analyses in both groups. The mean age at the time of surgery was 62 and 61 years old in the ASR and BHR groups, respectively. The ratio of male to female patients over both groups was 3:1. Acetabular inclination in the two groups correlated to femoral neck narrowing and was found to be significant. This study showed comparable thinning of the femoral neck between the two well established resurfacing systems with no linear correlation up to 4 years post-operatively and no clinically or radiographically significant sequelae in the short term to this bone loss. However, the reported significance in certain measured parameters to this osteolytic phenomenon does require continued monitoring for the long-term survival of resurfaced implants.

Conflict of interest: None

Disclosure: No benefit/s of any form has or will be received from a commercial entity related either directly or indirectly with this research.

\section{MORTALITY AND MORBIDITY RISK FACTORS IN REVISION ARTHROPLASTY}

\author{
N. Bandorf, D. Lui, P. Riordan, O. Jaweesh, B. Duru, D. Bennett
}

Department of Trauma \& Orthopaedics, Mayo General Hospital, Castlebar, Department of Orthopaedics, Galway Clinic, Galway, Ireland

Background: Projections indicate that by 2030 the number of revision THA's will increase $137 \%$ and the number of revision TKA's $601 \%$. The rate of revision THA is increasing in a population whose longevity increasingly outlives the lifespan of prosthetic implants. 
Methods: Prospectively collected data. 102 consecutive patients (undergoing 123 procedures) underwent revision hip arthroplasty by a single surgeon from June 2005 to June 2009 in 2 institutions. We investigated indication for revision surgery including aseptic loosening, infection, dislocation, peri-prosthetic fracture and implant failure. Demographic data including gender, age, ASA, co morbidities and reason for failure were recorded. Our outcomes measured were mortality, dislocation, infection, fracture and thrombotic events.

Results: Median age $=73.5$ years (range from 42 to 87 years). Median $\mathrm{ASA}=2$. Indications for revision were in $62 \%$ aseptic loosening, $15 \%$ infection, $11.5 \%$ fracture, $10.6 \%$ dislocation and $0.9 \%$ implant failure. Co-morbidities data showed: $28.4 \%$ cardiac, $4 \%$ respiratory and $6.8 \%$ diabetic. 5 significant complications in 4 patients accounted for a $3 \%$ complications rate. No mortality.

Conclusion: Compared to primary surgery, revisions are technically more demanding with longer operative times and increased blood loss making these procedures more risky for elderly patients. Revision arthroplasty surgery is effective in patients over 75 years and is effective in relief of pain and restoration of function as comparable to those younger. We identified potential risk factors. The single largest reason for revision arthroplasty in this series was aseptic loosening followed by infection and then fracture or instability in keeping with literature. Conflict of interest: No benefits in any form have been received or will be received from a commercial party related directly or indirectly to the subject of this article.

Disclosures: None

\section{THE IMPACTION FREQUENCY AND THRESHOLD FORCE REQUIRED FOR FEMORAL IMPACTION GRAFTING IN REVISION HIP ARTHROPLASTY-A HUMAN CADAVERIC BIOMECHANICAL STUDY}

\author{
F. Cummins, P. Kenny, D. Kelly
}

Cappagh National Orthopaedic Hospital, Finglas, Dublin, Department of Bioengineering, Trinity College Dublin

\footnotetext{
Summary: A laboratory based study investigating fracture forces and implant subsidence rates in embalmed human femurs undergoing impaction grafting.

Methods: Human femurs were harvested from cadavers for destructive impaction testing. An initial group of femurs underwent destructive impaction testing, using the impaction grafting technique as described by Gie et al., modified, allowing increasing, controlled impaction forces to be applied until femoral fracture occurred.

A second group of embalmed human femurs underwent impaction bone grafting at constant force, with varied impaction frequencies.. An Exeter stem was cemented into the neo-medullary canals. These constructs underwent subsidence testing simulating the first 2 months post-operative weight-bearing.

Results: In a group of 17 femurs, none fractured below a $0.5 \mathrm{kN}$ impaction force. $82 \%$ of the femurs fractured at or above $1.6 \mathrm{kN}$ of applied force. No massive implant subsidence occurred in the second group of 8 femurs, all undergoing femoral impaction grafting at $1.6 \mathrm{kN}$. There was no correlation between implant subsidence and frequency of impaction. Average subsidence was $3.2 \mathrm{~mm}$.

Conclusions: It is possible to calculate a force below which no fracture occurs in the embalmed human femur undergoing impaction grafting. Increasing impaction frequency, at constant force, doesn't decrease rates of implant subsidence.

Conflict of Interests: None

Disclosures: None
}

\section{THE EFFECT OF ANTICOAGULANTS ON THE OUTCOME OF TOTAL KNEE ARTHROPLASTY}

\author{
I. Radovanovic, J. Queally, S. Bahari, J. Sproule, S. Henari,
} J. McElwain

Adelaide and Meath National Children's Hospital, Tallaght, Dublin, Ireland

The purpose of this study was to determine the effect coumadin and clopidogrel on the outcome of total knee arthroplasty.

We conducted a matched case study on 5 years prospective collective data of patient undergoing total knee arthroplasty, who were on coumadin or clopidogrel pre operatively. Demographic data, type of anticoagulant, type of surgery, ASA level, intra-operative and postoperative blood loss, post-operative complications, and length of stay. Patients were matched to a control group who were not on anticoagulants.

30 patient on long term anticoagulation were identified. Mean operating time was $129 \mathrm{~min}$ compared to $104.5 \mathrm{~min}$ in the control group. Sub analyzing the data, patients on clopidogrel had a mean operating time of $120 \mathrm{~min}$ compared to $133 \mathrm{~min}$ for those on coumadin. The mean intra operative and post operative blood loss was $223.5 \mathrm{mls}$ and $538 \mathrm{mls}$, respectively, in the anticoagulant group while the control group blood loss was $67.5 \mathrm{mls}$ and $472 \mathrm{mls}$, respectively. Anticoagulated patients had a high incidence of blood transfusion (10/ 30 to 4/30). Anticoagulated patients had longer inpatients stayed compared; (20 compared to 10.8 days).The rate of infection for the anticoagulant group was $10 \%$ while the control group was $0.04 \%$.

Long term anticoagulant therapy increased length of operating, intra operative and post operative blood loss which may require post operative blood transfusion. These patients are also at risk of increase inpatient stay and wound ooze that put them at risk of infection. This translated as an increase cost for a routine knee arthroplasty procedure. Conflict of Interest: None

Disclosures: None

\section{THE USE OF CIRCULAR EXTERNAL FIXATORS FOR FRACTURE STABILISATION AND MANAGEMENT OF FRACTURE NON- UNION. A REVIEW OF 15 YEARS EXPERIENCE}

\author{
B. J. O’Neill, D. P. Moore \\ The Adelaide \& Meath Hospital Incorporating the National \\ Children's Hospital Tallaght, Dublin 24, Ireland
}

Background: Ilizarov and Taylor Spatial Fixators are commonly used in the management of complex fractures and in the management of fracture non-union at our institute. We aim to review 15 years of circular frame use by a single surgeon to assess the incidence of successful treatment of fractures and fracture non-unions.

Methods: We retrospectively reviewed the case notes, theatre lists, and radiology records of all patients who had been treated with a circular fixator over the past 15 years. We identified 134 procedures in 114 patients where a circular fixator was used for fracture stabilisation or treatment of non-union of fracture. We documented the length of time each frame was in situ and the outcome of treatment. Results: We identified 60 fractures in 54 patients and 74 fracture nonunions in 73 patients. Of the fracture non-unions 20 were known to be infected and 74 were considered sterile. Average length of time with a fixator in -situ was 243 days for the fracture group, 301 days for the sterile non-union group, and 343 days for the infected non-union 
group. 50 of $60(83.3 \%)$ fractures united satisfactorily and four malunited. Five fractures developed sterile non-union and one developed infected non-union. Of 54 sterile non-unions 42 united (77.8\%), five with significant mal-union. Three were thought to have united but refractured after removal of frame. Eight failed to unite and one patient died of unrelated cause with fixator in situ. Of 20 infected non-unions, fourteen united $(70 \%)$, three with significant mal-union. One nonunion was thought to have healed but re-fractured when the fixator was removed. Four remained infected and failed to unite and one failed due to soft tissue complications not associated with the fixator. Conclusions: Circular External Fixators are an appropriate method of treatment for complex fractures and fractures that have failed to unite when treated with alternative fixation devices.

Conflict of interest: None

Disclosures: None

\section{SESSION 7: ORTHOPAEDIC SESSION}

\section{THE MRSA PREVALENCE IN TRAUMA PATIENTS ADMITTED FROM NURSING HOMES; THE NEED FOR GLYCOPEPTIDE PROPHYLAXIS}

\author{
C. Green, D. Hennessey, C. Fitzpatrick, L. Fenelon, K. O'Rourke
}

Department of Trauma and Orthopaedic Surgery,

St. Vincent's University Hospital

Postoperative surgical site infection (SSI) is associated with increased morbidity. The use of prophylactic antibiotics is effective in reducing the rate of SSI. Guidelines recommend the use of a cephalosporin in the general population and a glycopeptide in the meticillin resistant staphylococcus aureus (MRSA) colonised patient. However, in the trauma patient MRSA status is usually unknown at the time of admission and surgery.

Aim: The aim of this study was to determine if the incidence of MRSA colonisation in trauma patients admitted from a nursing home warrants the use of glycopeptide antibiotic.

Method: A retrospective study of all patients admitted with hip fractures to tertiary referral centre was performed. The MRSA status of patients admitted with hip fractures from long term care (LTC) facilities was determined.

Results: 405 patients were swabbed for MRSA. 125 patients were admitted from LTC, 42 were carriers of MRSA (33.6\%). Only 11 $(3.9 \%)$ of patients admitted from home were as carriers of MRSA, $P<0.001$.

Conclusion: There is a significant prevalence of MRSA colonisation in trauma patients admitted from LTC. As a result it is now the practice of this department to use a glycopeptide antibiotic as prophylaxis when the MRSA status is unknown preoperatively. Conflict of interest: None

Disclosures: None

\section{FRACTURES OF THE HUMERAL DIAPHYSIS: AXIAL DISTRACTION AND NON-UNION}

S. A. Brennan, K. Ryan, R. J. Walls, D. Murphy, P. Kenny, P. Keogh, S. O'Flannagan
Connolly Hospital, Blanchardstown, Dublin, Ireland

Conservative management remains the gold standard for many fractures of the humeral diaphysis with union rates of over $90 \%$ often quoted. Success with closed management however is not universal. A retrospective review of all conservatively managed fractures between 2001 and 2005 was undertaken to investigate a suspected high non-union rate and identify possible causes. Risk factors identified included a high degree of distraction at first application of hanging cast, late conversion to functional brace and frequent prescription of NSAIDS. Changes to practise and re-audit over the following two year period resulted in a dramatic decrease in the non-union rate from 39.2 to $4.7 \%$. Axial distraction is a causal factor in the development of non-union of fractures of the humeral diaphysis.

Conflict of interest: None

Disclosures: None

\section{SELECTIVE ULTRASOUND SCREENING FOR DEVELOPMENTAL DISPLASIA OF HIP (DDH). EFFECTIVENESS OF ONE STOP DDH CLINIC}

\author{
G.A. Naqvi, S. A. Malik, O. Adamec
}

Department of Orthopaedic, Our Lady of Lourdes Hospital, Drogheda, Co. Louth, Republic of Ireland

Early diagnosis and treatment is the key for successful management of developmental dysplasia of hip (DDH). The natural acetabular growth and improvement of coverage is directly dependent on early rectification of biomechanics in dysplastic hip and the knowledge of soft tissue anatomy, provided by early ultrasound (US) is the main contributing factor in successful conservative treatment of DDH.

The aim of this study is to prove the role of ultrasound in paediatric orthopaedic clinic for early diagnosis and treatment of DDH in one stop clinic.

A program of ultrasound screening of "at risk" babies was started in our department since July 2008 as a one stop DDH clinic. All newborn infants with the risk factors of DDH and any abnormal finding were included for screening. During July 2008 to June 2009 (1 year), 332 infants (664 hips) were screened for DDH using clinical examination and ultrasonography by orthopaedic consultant using Graf's technique. Mean age was 64 days. Treatment of dysplastic and dislocated hips was started immediately in the form of pavlic harness. Dislocated or instable joints are followed-up weakly until stabilised while dysplastic joints on a monthly bases until normalising ultrasound parameters.

Out of 664(332 infants) hips examined, 560 (84.3\%) were labelled as normal on ultrasonography. $104(15.6 \%)$ hips in 70 patients were diagnosed as dysplastic or dislocated. Clinical examination only detected 27 patients of DDH correctly out of 70 , sensitivity of $38 \%$, specificity of $100 \% .68$ patients were successfully treated with pavlic harness for average duration of 3 months while 2 required traction to achieve stable reduction.

Hip ultrasound screening in babies with risk factors is effective in early diagnosis and treatment of those with DDH and significantly reduces the duration of conservative treatment as well as the need for surgical intervention.

Conflict of interest: None

Disclosure: None 


\section{ARTHROSCOPIC MANAGEMENT OF SOFT TISSUE ANKLE IMPINGMENT}

\author{
S.A. Brennan, K. Ryan, F. Rahim, J. Dowling, S. Kearns \\ University College Hospital Galway, Ireland
}

Forty-one ankle arthroscopies were performed for soft tissue impingement between April 2007 and April 2009. There were 26 men and 15 women and the mean age was 30.1 years. Arthroscopy was performed on average 21 months after injury. The Visual-AnalogueScale Foot and Ankle (VASFA) score and Meislins' criteria were used to assess response to treatment. The mean pre-operative VASFA score was 44.5 . This increased to 78.3 postoperatively $(\mathrm{p}<0.0001)$. According to Meislins criteria there were 34 good or excellent results, 5 fair and 2 poor results. Pre-operative magnetic resonance imaging (MRI) was useful in detecting tears of the anterior talofibular ligament and excluding osteochondral defects; however synovitis and soft tissue impingement was under-reported. Arthroscopy is an effective method for the diagnoses and treatment of soft tissue impingement of the ankle joint.

Conflict of interest: None

Disclosures: None

\section{A RISK ANALYSIS FOR THE OPERATIVE VERSUS NON-OPERATIVE MANAGEMENT OF POSTERIOR MALLEOLAR FRAGMENTS AT 5 YEARS}

E. Cullen ${ }^{1}$, A. O’Conghaile ${ }^{1}$, R. Flavin ${ }^{1}$, A. Simpkin ${ }^{2}$, D. Bergin ${ }^{3}$, D. O'Keefe ${ }^{3}$, S. Kearns ${ }^{1}$

Department of Orthopaedics, Merlin Park Hospital, Galway, Ireland $^{l}$, Health Research Board, Clinical Research Facility, NUI, Galway, Ireland ${ }^{2}$, Department of Radiology, Merlin Park Hospital, Galway, Ireland ${ }^{3}$

The incidence of post-traumatic osteoarthritis (OA) of the ankle following a fracture is significant, in some series approaching thirtyseven percent. There is controversy about the correlation between biomechanical and clinical studies. The purpose of this study was to determine the risk of $\mathrm{OA}$ in ankle fractures with a posterior malleolar fragment and whether this depended specifically on the operative treatment of the fragment. Two cohorts were selected and best matched for age, sex and fracture pattern. Both groups had an open reduction and internal fixation of their fracture, the division being whether the posterior malleolus was fixed operatively or not. A total of 45 patients had fixation of the posterior malleolar fragment from January 2000 to December 2007 at a tertiary referral centre. The mean age at the time of surgery was 56 and 52 in the fixed and control groups, respectively. The ratio of male to female patients was 1:4 across both groups. Patients were assessed at follow-up using the American Orthopaedic Foot and Ankle Society, Foot and Ankle Outcome and Visual Analogue Scoring systems. Radiographic analysis was performed via an osteoarthritic-score. The key outcome was a statistically significant negative difference in the correlated risk ratio to clinical and radiographic osteoarthritis in the fixed to control groups. The risk to post-traumatic OA in our study following a posterior malleolar elemental fracture was significantly reduced following fixation of the fragment, regardless of the fragment size, in a case matched cohort group at a mean followup of 5 years.

Conflict of interest: None
Disclosure: No benefit/s of any form has or will be received from a commercial entity related either directly or indirectly with this research.

\section{RhBMP-2 USE IN LUMBAR FUSION SURGERY IS ASSOCIATED WITH BETTER LEG PAIN SCORES AT LATE FOLLOW UP}

\author{
F.E. Rowan ${ }^{1}$, N. O'Malley ${ }^{2}$, A. Poynton ${ }^{1,2}$ \\ Department of Orthopaedics and Spinal Surgery, Mater Private
} Hospital, Dublin 7, Ireland ${ }^{1}$, Department of Orthopaedics and Spinal Surgery, Cappagh National Orthopaedic Hospital, Dublin 11, Ireland $^{2}$

Introduction: Recombinant human bone morphogenic protein-2 (rhBMP-2) eliminates the need for iliac crest bone graft and has superior fusion rates in anterior interbody fusion1. Peri-rhBMP-2 oedema is a proposed cause of neuropathic leg pain in posterolateral lumbar fusion.

Aim: To compare the incidence of early and late post-operative leg pain between rhBMP-2 treated patients and controls following posterolateral lumbar fusion.

Method: A single surgeon, multi-centre elective practice was retrospectively reviewed over a 4-year period. All rhBMP-2 treated patients were included. There were 64 and 40 patients in the rhBMP-2 treated and control group, respectively.

Results: Sixty-four rhBMP-2 treated patients and 40 controls were included. Pre-operative demographics and diagnoses were similar. Inter-body cages were used equally. Mean rhBMP-2 and nonrhBMP-2 cohort follow up was $13.0 \pm 11.4$ and $5.8 \pm 4.1$ months, respectively. Immediate post operative leg pain incidence was 25 and $12.5 \%$ in the rhBMP-2 and non- rhBMP-2 groups, respectively. At late follow up, leg pain incidence was 5 and $10.2 \%$ in rhBMP-2 and non- rhBMP-2 groups, respectively. Oswestry Disability Indices were lower (4 vs. 18) in rhBMP-2 treated individuals at late follow up. MRI evidence of mechanical cause for leg pain post-op was $7.8 \%$ in rhBMP-2 treated individuals and 5\% in controls.

Conclusion: In primary lumbar fusion surgery, early post operative leg pain is higher in patients treated with rhBMP-2 without associated differences in radiologically determined mechanical cause. Postoperative leg pain is significantly reduced at late follow up in rhBMP2 treated patients $(p<0.002)$. RhBMP-2 use is associated with better leg pain scores at late follow up.

Conflict of interest: None

Disclosures: None

\section{ANTERIOR CERVICAL DISC REPLACEMENT: EARLY CLINICAL RESULTS}

\author{
S. Munigangaiah, K. C. Cronin, J. P. McCabe \\ Department of Trauma and Orthopaedic Surgery, University College \\ Hospital Galway, Newcastle, Galway, Ireland
}

There have been limited published reports on the clinical results of cervical artificial disc replacement. Goffin et al. reported a $90 \%$ rate of good to excellent results at 1-2 years after Bryan disc replacement. Wigfield et al. reported a $46 \%$ improvement in pain and $31 \%$ improvement in disability 2 years after Prestige cervical disc replacement. 
The study was designed to determine whether new functional cervical disc prosthesis can provide improvement in the ability to perform activities of daily living, decrease pain and segmental motion.

All patients who had Prestige cervical disc replacement over last 2 years 3 months were identified. Oswestry disability index score and SF36 Quality of life instrument were administered to each patient. Site specific pain scores were obtained using visual analogue scale. Clinical and operative details were reviewed and correlated.

35 Patients underwent 48 Prestige cervical disc replacement during study period. There was 48,54 and $70 \%$ improvement in Oswestry disability index at 6 weeks, 6 months and 19 months follow-ups, respectively. There was significant improvements in both neck and arm pain during immediate post op period and during further follow-ups. There was one neck wound haematoma and one left sided Horner's syndrome as complications.

Anterior cervical disc replacement is a viable surgical alternative to fusion for cervical disc degenerative disease and herniation with preservation of motion and alignment without compromising clinical outcomes. At least 5 years follow-up will be needed to assess the long term functionality of the prosthesis and protective influence on the adjacent levels.

Conflict of interest: None

Disclosure: None of the contributing authors received any financial inducements from the manufacturers of medical implants connected with this research.

\section{A NOVEL TECHNIQUE FOR DETERMINING TRANSVERSE SKIN INCISION SITE FOR ANTERIOR CERVICAL SPINE SURGERY}

\section{Kennedy, M. Leonard, H. Heneghan, J.P. McCabe \\ Department of Trauma and Orthopaedic Surgery, University Hospital Galway, Ireland}

Background: The transverse skin incision for anterior cervical spine surgery is not extensile thus it must be made at the accurate level. The use of palpable bony landmarks is unreliable due to anatomical variations, and pre-operative fluoroscopy to identify the level takes up operating room time, increases the radiation dose to the patient and increases the overall cost of the operation.

Objective: To describe a simple, fast and inexpensive method of accurate transverse skin incision placement for anterior cervical spine surgery, and to report on its use in 54 consecutive adult patients.

Patients and methods: In each case a ratio was recorded on the lateral cervical spine radiograph based on the distance between the clavicle and mandible and the operative level, this was then applied to measurements on the patient's neck.

Results: Procedures performed consisted of a mix of discectomy and fusion, disc replacement and combinations of both. The operative level ranged from $\mathrm{C} 2-\mathrm{C} 3$ to $\mathrm{C} 7-\mathrm{T} 1$, the most common being $\mathrm{C} 5-\mathrm{C} 6$. Twenty-three patients had a single-level, 26 a two level and five a three level procedure, all cases were performed through one single transverse incision.

Conclusion: In conclusion we describe the highly successful use of a straightforward method for accurate level transverse skin incision placement for cervical spine surgery In no case was it necessary to radically extend or to make a separate incision. There were no cases where the wrong level was operated on.

Conflict of interest: There was no conflict of interest. All the authors disclose there was no financial or personal relationship with other people or organizations that could inappropriately influence our work. No grant or funding was received for the above study.

\section{OUTCOMES OF TRACTION SPLINTING OF CLOSED PROXIMAL PHALANGEAL FRACTURES}

\author{
S.M. O'Meara, D.T. Cawley, F.J. Shannon \\ Department of Orthopaedic and Trauma Surgery, Galway University \\ Hospital, Galway, Ireland
}

Proximal phalangeal fractures are caused by an injury to the dorsum of the hand. This usually causes volar angulation which is unstable when reduced. K-wiring or minifragment screws can damage the soft tissue envelope, can introduce infection and can loosen or displace. Traction splinting is not well described for these fractures. Functional and radiographic assessment of all patients with proximal phalangeal fractures treated with traction splinting. Theatre records were examined for relevant injuries over a 2 year period. These patients were then assessed using a QuickDASH score, a questionnaire specific to traction splinting and with pre-op, intra-op, post-op and follow-up radiographs. A total of seven patients were treated with traction splinting, all by the senior author (FJS). Clinical follow was 16 months (range 12-20). QuickDASH scores were 0, 0, 0, 0, 0, 2.5, 25/100. With regard to work $(n=6)$, all patients but one scored $0 / 100$ for disability with one patient describing mild work related difficulties. Those participating in sports/ performing arts $(n=6)$ scored $0 / 100$. There were no finger-tip pain or numbness issues. Finger length perception was satisfactory in all patients. The splint slipped in three patients, secondary to horse riding, showering and through scratching. Two patients reported being unable to fully flex or extend the affected digit (follow-up 18 and 20 months). Radiographic outcomes showed that traction achieved acceptable length restoration, with no angular deformities. Finger length was maintained in all but one patient who had a shortening of $3.2 \mathrm{~mm}$. Traction splinting is a non-invasive, safe and inexpensive method of treating proximal phalangeal fractures. Results of our follow-up study show excellent functional and radiographic outcomes with minimal long term morbidity for this treatment option.

Conflict of interest: None to declare

Disclosures: None to declare

\section{FREE HAND VERSUS NOVEL SPECIALISED JIG GUIDANCE FOR THE PASSING OF INTRA- MEDULLARY WIRES IN OLECRANON FRACTURE FIXATION: A COMPARATIVE STUDY}

\author{
A. Gheiti, D.C. Molony, J. Kennedy, J.H. Mullett \\ Cappagh National Orthopaedic Hospital, Finglas, Dublin 11, \\ Republic of Ireland
}

Background: The treatment of olecranon fractures frequently involves the use of tension band fixation. Although associated with high union rates, this method has a high incidence of morbidity associated with soft tissue compromise and limitation of range of movement requiring frequent re-operation for removal of metal.

Objectives: We describe the use of a simple jig to ensure intramedullary placement of longitudinal K-wires and compare the accuracy of placement of wires using this device with the traditional free hand method.

Methods and materials: 10 orthopaedic surgical trainees passed 2 longitudinal $\mathrm{k}$ wires into synthetic ulnae. This was done using the free hand method and then plastic jig. The ulnae were then sectioned to identify the position of the wires relative to the intramedullary canal 
Results: Of the free hand wires, only 11 of 20 were found to be intramedullary versus 20 of 20 in the group passed using the jig $(p<0.001)$. The mean distance from the center of the ulnar canal was $6.5 \mathrm{~mm}$ in the freehand group (range 1-18 mm, SD $6.1 \mathrm{~mm}$ ) and $1.6 \mathrm{~mm}$ in the O-Jig group (range 1-2 $\mathrm{mm}, \mathrm{SD} 0.5 \mathrm{~mm}$ ). The difference in the mean distance from the centre was $4.9 \mathrm{~mm}$ which was significant $(p<0.001)$

Conclusions: We found the distance from the centre of the medullary canal, the range and standard deviations of wire positions to be significantly more precise when the jig was used.

Conflict of interest/Disclosures: H. Mullet, has interest through share captial in the company (SOTA orthopaedics), no conflict with other authors.

\section{RADIOLOGICAL OUTCOMES OF DISTAL RADIUS EXTRA-ARTICULAR FRAGILITY FRACTURES TREATED WITH EXTRA-FOCAL KIRSCHNER WIRES}

\author{
C. Kennedy ${ }^{1}$, M.T. Kennedy ${ }^{2}$, D. Niall ${ }^{2}$, A. Devitt ${ }^{1}$ \\ Department of Orthopaedic Surgery, University College Hospital, \\ Galway, Republic of Ireland ${ }^{l}$, Department of Orthopaedic Surgery, \\ Midland Regional Hospital, Tullamore, Co Offaly, Republic of \\ Ireland $^{2}$
}

Introduction: The classical Colles fracture (extraarticular, dorsally angulated distal radius fracture) in patients with osteoporotic bone is becoming increasingly more frequent. There still appears to be no clear consensus on the most appropriate surgical management of these injuries.

Methods: We retrospectively analysed 72 consecutive cases of Colles fractures treated with interfragmentary $\mathrm{K}$-wire fixation, in female patients over 60 years of age, in two orthopaedic centres, under the care of 12 different orthopaedic surgeons. We correlated the radiographic distal radius measurements (ulnar variance, volar tilt, and radial inclination) at the pre-operative and intra-operative stages with the final radiographic outcome.

Result: Mean dorsal angulation was $21^{\circ}$ at time of presentation. Closed reduction significantly improved fracture position to a mean of $2.7^{\circ}$ volar angulation $(p<0.05)$. Mean angulation at time of $\mathrm{k}$-wire removal was $1.6^{\circ}$ dorsal, this was not significant in comparison to post reduction measurements $(p<0.05)$. Mean ulnar variance at time of presentation was $2.5 \mathrm{~mm}$ (range 7.4 to -4.2 ). Reduction improved fracture displacement to a mean of $0 \mathrm{~mm}$, which was statistically significant $(p<0.05)$. Mean ulnar variance at time of $\mathrm{k}$-wire removal was $2.4 \mathrm{~mm}(p<0.05) .56 .8 \%$ of cases demonstrated radial shortening of $2 \mathrm{~mm}$ or more.

Conclusion: In female patients over 60 years of age, the best predictor of radial length, when $\mathrm{K}$-wire fixation is to be used, is the radial length prior to fracture reduction. Thus if there is radial shortening visible in the initial radiographs as measured in terms of ulnar variance, one should consider a method of fixation other than interfragmentary K-wires.

Conflict of interest: There was no conflict of interest. All authors disclose there was no financial or personal relationships with other people or organizations, that could inappropriately influence our work. No grant or funding was received for the above study.
SESSION 8: ANAESTHESIA SESSION

\section{COMPARISON OF ANALGESIC REQUIREMENT IN FIRST 24 H FOLLOWING TOTAL KNEE ARTHROPLASTY IN PATIENTS WITH OR WITHOUT PERIARTUCLAR CHIROCAINE ADMINISTRATION; RETROSPECTIVE ANALYSIS OF CASE NOTES}

\author{
A. Memon, S. Umar, B. Suleman, R. Gul, J. Harty, M. Dolan \\ St Mary's Orthopaedic Hospital, Cork University Hospital, Cork, \\ Ireland
}

Background: Post operative analgesia is an important part of Total Knee Arthroplasty (TKA) to facilitate early mobilisation and patient satisfaction. We investigated the effect of periarticular infiltration of the joint with chirocaine local anaesthetic (LA) on the requirement of analgesic in the first $24 \mathrm{~h}$ period post-operative.

Methods: Retrospective analysis of case notes was carried out on 28 patients, who underwent TKA by two different surgeons. They were divided into two groups of 14 each; who did and did not receive the LA infiltration respectively. All patients were given spinal morphine (162 mcg $r$ : 150-200). Analgesic requirement was assessed in terms of the amount of paracetamol, morphine, diclofenac, oxynorm and tramadol administered in $24 \mathrm{~h}$ post-operative including the operating time.

Results: Following results were obtained from patients receiving LA infiltration vs no infiltration: Morphine; 70 versus $200 \mathrm{mg}$, Paracetamol; 60 versus $58 \mathrm{gm}$, Diclo fenac; 1,650 versus $1,050 \mathrm{mg}$, Oxynorm; 40 versus $80 \mathrm{mg}$, Tramadol; 200 versus $400 \mathrm{mg}$. Average length of stay (LOS) was 6 days ( $r$ : 3-8) in both groups.

Conclusion: From this study it may be concluded that periarticular LA infiltration reduces the requirement of morphine in first $24 \mathrm{~h}$ by almost $1 / 3$ rd. The amount of tramadol and oxynorm was also halved in LA infiltrated group although the requirement of paracetamol remained the same. LA infiltrated group received almost 1.5 times more Diclofenac as compared to the non infiltrated group. The LOS was not affected by the administration of LA.

Conflict of interest: None

Disclosures: None

\section{INFLUENCE OF BAROMETRIC PRESSURE ON PAIN SEVERITY IN END STAGE OSTEOARTHRITIS}

\author{
S.A. Brennan ${ }^{1}$, K. Ryan ${ }^{1}$, T. Harney ${ }^{1}$, C. Gormley ${ }^{2}$, N.A. Rahim ${ }^{1}$, \\ F. Shannon
}

University College Hospital Galway, Ireland ${ }^{l}$, School of Mathematical Science, University College Dublin ${ }^{2}$

Patients often attribute increasing pain in an arthritic joint to changing weather patterns. Studies examining the impact of weather on pain severity have yielded equivocal and sometimes contradictory results. The relationship between subchondral pseudocysts and the role they play in this phenomenon has not been explored. Fifty-three patients with end stage osteoarthritis of the hip completed daily pain severity visual analogue scores over a 1 month period. Radiographs were reviewed to determine the presence of pseudocysts. Data pertaining to precipitation, atmospheric pressure and temperature was collected from the most proximate weather station. A generalized linear mixed 
model was used to explore the relationship between weather variables, cysts and pain severity. Pain levels increased as a function of absolute change in atmospheric pressure from 1 day to the next. Precipitation, temperature and the presence of subchondral pseudocysts were not shown to influence pain severity. This data supports the belief held by many osteoarthritic patients that changing weather influences their pain severity.

Conflict of interest: None

Disclosures: None

\section{ILIOLUMBAR SYNDROME: SONOGRAPHIC ANATOMY AND INJECTION TECHNIQUE}

\author{
V. Alexiev, D. Harmon \\ Department of Anaesthesia and Pain Medicine, \\ Mid-Western Regional Hospital, Dooradoyle, Limerick, Ireland
}

Iliolumbar syndrome is a frequently unrecognized cause of sciatica that results from inflammation, sprain, or tear of the iliolumbar ligament. This ligament bridges the transverse process of the fifth lumbar vertebra and iliac crest. People who lift heavy objects while rotating the back are especially prone to the iliolumbar syndrome. Patients complain of pain that varies from a constant, dull ache aggravated by activity to extreme severity. Pain is usually localized to the posterior portion of one or the both iliac crests, but may spread across the iliolumbar region. It can lead to referred pain in the groin, the pelvis, the hip, the back and even the testicular, vaginal and rectal areas. There is tenderness to palpation of the iliac crests. There is pain on the affected side with lateral bending of the lumbar spine to the opposite side. Treatment includes physiotherapy and local injection. Local injection should relieve pain, thus confirming the diagnosis. If the presumptive diagnosis is correct, the above tests should become negative. Previously a blind injection technique was used [1]. Here we report for the first time the sonographic anatomy of the iliolumbar ligament and its injection technique. Ultrasound improves block accuracy and thus diagnosis. Ultrasound offers other advantages. Prolotherpy which has been used as a treatment can be guided by ultrasound.

(1) Naeim F, Froetscher L, Hirschberg GG (1982) Treatment of the chronic iliolumbar syndrome by infiltration of the iliolumbar ligament. West J Med 136(4):372-374.

Conflict of interest: None

Disclosures: None

\section{AUDIT ON PRE-PREPARATION OF "EMERGENCY DRUGS": FINANCIAL IMPACT, AWARENESS OF COSTS AND PRACTICE OF PRE-PREPARATION}

\author{
J. Steiner ${ }^{1}$, C. Maharaj ${ }^{2}$, W. O’Brien ${ }^{1}$ \\ Mid-Western Regional Hospital, Limerick, Ireland ${ }^{I}$, University \\ Hospital, Galway, Ireland ${ }^{2}$
}

Introduction: Considered routine practice, there are no national guidelines regarding predrawing of emergency drugs. This audit examined the cost of this practice, the awareness of costs involved and looked into factors surrounding pre-preparation of emergency drugs. Methods: The initial questionnaire required the Anaesthetic NCHD to highlight which drugs were drawn up from a list of commonly predrawn drugs. Prepared emergency drugs which were actually used during the day were recorded. The second part looked into time, cost and cost awareness of predrawing emergency drugs and asked about personal opinions regarding this practice.

Results: 7 surveys representing one week were recorded. The average cost for predrawn emergency drugs and materials used was $€ 3.05$ (24\%) and $€ 9.70(76 \%)$ for unused.

The second survey revealed unawareness of costs involved. $20 \%$ reported they had been instructed regarding preparation of emergency drugs, $90 \%$ reported that the cost of pre-preparing drugs had never been discussed.

Ten percent felt it was safe not to routinely draw up emergency drugs while $90 \%$ reported they had used pre-prepared drugs in an emergency.

Discussion: The majority of pre-prepared emergency drugs are wasted on a daily basis indicating that at our hospital $€ 50$ is wasted on a daily basis due to this practice.

The projected financial impact is significant: assuming that all hospitals in Ireland have on average five theatres running per day this translates into annual wastage of approximately $€ 1,000,000$. Options to improve or change this practice should be discussed nationally and practice guidelines should be implemented.

\section{References:}

Gurung AM, Tomlinson AA (2004) Pre-preparation of succinylcholine: significant waste for questionable benefit. Anaesthesia 59:211215

Webster CS, Merry AF, Ducat CM (2001) Safety, cost and predrawn emergency drugs. Anaesthesia 56:818-820

Conflict of interest: The authors wish to state there was no conflict of interest.

Disclosures: None

\section{SAFER SURGERY: HOW ACCURATE ARE WE AT PREDICTING INTRAOPERATIVE BLOOD LOSS?}

\author{
C. Egan, J.G. Solon, D.A. McNamara \\ Department of Surgery, Beaumont Hospital, Beaumont, Dublin 9, \\ Ireland
}

The WHO Surgical Safety Checklist requires preoperative estimation of expected blood loss. Our aim study was to assess the accuracy of surgeons and anaesthetists in predicting intraoperative blood loss.

A 6 week prospective study of elective and emergency operations was performed. Surgical and anaesthetic consultants and registrars estimated expected blood loss preoperatively. This was compared with nursing measurements of actual blood loss.

One hundred and sixty-six cases were prospectively studied. Overall, surgeons were more accurate than anaesthetists. The mean percentage variance from actual blood loss among consultant surgeons was $141 \%$ compared with $206 \%$ for consultant anaesthetists. When the consultant surgeon scrubbed for the operation the surgeon was more accurate, otherwise the anaesthetist was. In emergency cases anaesthetists were significantly more accurate at estimating blood loss than surgeons. There was no difference in the groups if the patient was on anti-coagulation or anti-platelet therapy. Colorectal surgeons were most accurate at predicting blood loss, in $30 \%$ of cases predicting to within $10 \%$ of actual loss, followed by neurosurgeons and breast surgeons. ENT and urology surgeons were the least accurate.

We have shown that consultant surgeons were most accurate in predicting blood loss. This highlights the benefits of specialisation and experience in predicting adverse events in elective surgery. However, the opposite was shown in emergency cases. 
Conflict of interest: None

Disclosures: None

\section{ASSESSMENT OF BALANCE WITH SHOULDER STABILIZATION SLING USING COMPUTERIZED DYNAMIC POSTUROGRAPHY}

\author{
A. Memon ${ }^{1, *}$, D. Lui ${ }^{2}$, H. Mullett ${ }^{2}$, S. Kwan ${ }^{3}$ \\ Cork University Hospital ${ }^{1}$, Beaumont Hospital Dublin, Ireland ${ }^{2}$, \\ Royal College of Surgeons Ireland ${ }^{3}$
}

Introduction: Computerized Dynamic Posturography (CDP) is NASA deigned software used to detect balance and stability impairments. CDP is a novel and objective method to assess balance in people wearing a sling. Hypothesis: Wearing a sling diminishes balance

Methods: 36 healthy adults aged 20-35 years were randomly divided into two groups of 18 into each Dominant Hand (DH) and Non Dominant Hand (NDH). Shoulder stabilization Sling (SSS) applied to DH or NDH Groups. The balance was assessed in terms of Sensory Organization Test (SOT), Motor Control Test (MCT) and Adaptation Test (ADT). The sum of both DH + NDH scores compared with the standard composite norms. The groups $\mathrm{DH}$ versus $\mathrm{NDH}$ were also compared.

Results: Wearing a sling led to $21.87 \%$ imbalances on testing. Slings on the dominant hand (DH) led to $18.4 \%$ imbalances versus $25.34 \%$ in the non-dominant hand (NDH). The absolute number of falls stood at 5 versus 10 in $\mathrm{DH}$ and $\mathrm{NDH}$ respectively. Overal $131.25 \%$ of individuals had composite score of less than normal on equilibrium testing. Quality factor on active force latency test reveals; Backwards: DH sling: Right 2.81, Left 2.85. NDH: Right 2.99, Left 3.19. Forwards: DH sling: Right 3.46, Left 3.68. NDH: Right 3.44, left 3.48

Conclusions: We conclude that the balance of an individual is affected with the application of SSS. $31 \%$ of the individuals had abnormal equilibrium score with $21.87 \%$ imbalances. Individuals with the sling on NDH had 50\% more falls than DH, which might seem counter intuitive. The quality factor was lower on backward translations in individuals with sling on DH. In conclusion we support our hypothesis. SOT \& MCT demonstrated that balance is affected with the application of a sling and its more diminished if the sling is applied on the NDH.

Conflict of interest: None

Disclosures: None

\section{USE OF PUMPLESS EXTRACORPOREAL INTERVENTIONAL LUNG ASSIST DEVICES (PEILADs) IN PATIENTS WITH H1N1 TYPE A INFLUENZA}

\author{
P. Johnson, A. Westbrook \\ Intensive Care Unit, St James's Hospital, Dublin 8, Ireland
}

The recent H1N1 pandemic is causing severe ARDS in some patients. PEILADs have been used in two of our patients with improvements in hypercapnia and acidosis.

$\mathrm{Mr} \mathrm{V}$, a morbidly obese 30-year-old gentleman developed severe H1N1 ARDS. He was started on high frequency oscillatory ventilation (HFOV) but rapid development of pneumothoraces, pneumomediastinum, and worsening haemodynamic instability ensued. Extracorporeal Membrane Oxygenation (ECMO) was not available. The right femoral artery and vein were cannulated percutaneously, but poor flow necessitated transfer to theatre for line manipulation by vascular surgeons under fluoroscopy. PEILAD then provided improved $\mathrm{pH}$ and $\mathrm{pCO} 2$. After 2 weeks the arterial line became dislodged. There was significant blood loss, a pseudo-aneurysm occurred at the site and a haematoma developed.

Mr O'R, a 57-year-old gentleman with multiple myeloma developed ARDS from H1N1 flu and became progressively difficult to ventilate. PEILAD was instituted instead of progression to HFOV. This was successful in improving his $\mathrm{pH}$ and $\mathrm{PCO} 2$ and the femoral lines remained in situ until they became uneventfully dislodged.

PEILADs appear to offer improved carbon dioxide removal and enable utilisation of less aggressive ventilation strategies. They have been used as salvage therapy, but may be of benefit in preventing ventilator-induced lung injury when introduced earlier. This may be of benefit to patients with viral pneumonitis, especially during pandemics when alternative ECMO resources are overwhelmed.

These devices have been used successfully but our experience has demonstrated the importance of:

- staff education

- vigilance for potential complications

- support from vascular surgeons

Conflict of Interest: None

Disclosures: None

POSTER SESSION-SPONSORED BY SANOFI AVENTIS

\section{DIGIT RATIO (2D:4D) AS A PREDICTOR OF BREAST CANCER PREDISPOSITION}

\author{
A. Devine, J. Kelly, H. Heneghan, R. Dwyer, M. Kerin
}

Department of Surgery, National University of Ireland, , Ireland

Second to fourth digit ratio (2d:4d) is a sexually dimorphic trait, dependent on prenatal hormone exposure. A longer relative second digit to fourth digit indicates increased oestrogen prenatally. Females tend to have larger ratios than males in humans and mice. $2 \mathrm{~d}: 4 \mathrm{~d}$ has been shown to correlate with fetal growth, fertility, sexuality, risk taking, sporting ability, aggression, autism and cardiovascular disease.

Our aim was to determine if a relationship exists between digit ratio and breast cancer predisposition in humans and mice.

We enrolled 195 women in the study; 50 controls, 54 diagnosed with breast cancer younger than 40 , and 91 diagnosed older than 40 years. Photocopies of palmar aspects of right hands were obtained and digits measured independently by two persons using vernier calipers, with mean value used. 24 female athymic nude $\mathrm{Balb} / \mathrm{C}$ mice were injected with subcutaneous T47d cell mass and implanted with oestradiol pellets. Tumor development was monitored and once sacrificed, animal right hind limb was amputated, fixed and measured using digital software.

Our results show the digit ratio of the control group was 0.976 , breast cancer group over 40 was 0.98 and younger than 40 was 1.00. Digit ratio was a statistically significant predictor of breast cancer predisposition in the under 40 group $(p=0.000)$, and over 40 group $(p=0.003)$ compared to controls. There was no significant difference in ratio between both mice groups $(p=0.420)$. 
In conclusion, digit ratio (2d:4d) is a predictor of breast cancer predisposition in humans but not in mice.

Conflict of interest: None

Disclosures: None

\section{ABDOMINAL AORTIC ANEURYSMS MECHANICAL PROPERTY QUANTIFICATION USING ACOUSTIC RADIATION FORCE IMPULSE}

\author{
A. Tierney ${ }^{1}$, D. Dumont ${ }^{1}$, A. Callanan ${ }^{2}$, T. M. McGloughlin ${ }^{2}$ \\ Duke University, North Carolina, USA ${ }^{1}$, Centre for Applied \\ Biomedical Engineering Research (CABER), Department of \\ Mechanical and Aeronautical Engineering, Materials and Surface \\ Science Institute (MSSI), University of Limerick, Ireland ${ }^{2}$
}

The elastic properties of major arteries are of interest from both a physiological and patho-physiological point of view. An Abdominal Aortic Aneurysm (AAA) is a focal balloonlike dilation of the infrarenal aortic segment, greater than $50 \%$ of its normal diameter. The pathogenesis of AAA formation is characterised by a destruction of elastin and collagen in the arterial wall. These pathological changes are generally correlated with changes in tissue stiffness. Acoustic Radiation Force Impulse (ARFI) Imaging is a methodology which provides information about the local mechanical properties of bodily tissue. This study investigates the applicability of ARFI Imaging to indicate the mechanical properties of in vivo AAA.

An artificial aneurysm was created on the excised porcine aorta using elastase infusion and balloon dilation. The stiffness of the arterial wall was expressed in terms of the ARFI displacement and Hudetz incremental elastic modulus, Einc. There was very similar trends in the ex vivo aneurysm displacement versus Einc relationship, which allowed an average behaviour to be determined. Using the average behaviour of the ex vivo models, an estimation of the in vivo Einc could be calculated from ARFI displacements.

Average pressure-diameter relationships taken from literature were applied to the in vivo systole diameter to allow calculation of possible Einc for the in vivo case.

Comparison of the ARFI Einc estimation to the calculated Einc demonstrated a very close relationship, which demonstrates the possibility of using ARFI to quantify in vivo mechanical properties. This may provide a valuable insight into the AAA properties and provide an additional diagnostic aid.

Conflict of interest: None

Disclosures: None

\section{SERUM ANTIBIOTIC PROPHYLAXIS FOR TRANSRECTAL ULTRASOUND GUIDED BIOPSY OF PROSTATE - A PROSPECTIVE COMPARATIVE STUDY OF TWO REGIMES INVOLVING >1000 MEN}

G. Nason, R.P. Manecksha, I.M. Cullen, E. McEvoy, T.E.D.

McDermott, R. Flynn, R. Grainger, J.A. Thornhill

Department of Urology, Adelaide and Meath incorporating the National Children's Hospital, Dublin 24, Ireland

Introduction: Transrectal ultrasound guided biopsy of prostate (TRUS biopsy) is associated with a 5\% infection risk and $0.5 \%$ risk of septicaemia. While antibiotic prophylaxis is standard care, consensus regarding the optimum regime is less clear in the literature. We aimed to compare sepsis rates for two antibiotic prophylaxis regimes.

Methods: In 2008, 558 men had a TRUS biopsy receiving $400 \mathrm{mg}$ Ofloxacin stat at the time of biopsy and $200 \mathrm{mg}$ twice daily for 3 days thereafter. From January 2009, 625 men had a TRUS biopsy with Ofloxacin $200 \mathrm{mg}$ prescribed twice daily for 5 days but commencing $24 \mathrm{~h}$ prior to biopsy. All patients had 10-12 core biopsies using $18 \mathrm{G}$ needles. Symptomatic urinary tract infections and septicaemic incidence rates were quantified at follow up of results. Laboratory results concerning offending pathogens and antibiotic sensitivities were obtained.

Results: $20 / 558$ (3.6\%) of men had infective episodes in 2008 compared with $9 / 625(1.4 \%)$ in 2009. In 2008, of the 20 septic episodes, 8 had positive urine cultures, 6 had positive blood cultures with 4 having both blood and urine cultures positive and 5 with no growth isolated. In 2009, of the 9 infective episodes, 5 had positive urine cultures, 4 had a positive blood culture with 2 having both cultures positive and 2 with no growth isolated. $5 / 8$ men with positive cultures in 2008 isolated a ciprofloxacin-resistant $E$. coli and 2/8 men had $E$. coli sensitive only to Meropenem. In 2009, 2/3 positive cultures isolated resistant E. coli strains.

Conclusions: Commencing ofloxacin prophylaxis $24 \mathrm{~h}$ prior to TRUS biopsy was associated with a relative risk reduction in septic episodes of $39 \%$ in our institution. Furthermore, we are seeing an emergence of quinolone-resistant and multi-resistant E.coli.

Conflict of interests: None

Disclosures: None

\section{SITE DISTRIBUTION OF MELANOMA. A RETROSPECTIVE STUDY EXAMINING THE RELATIONSHIP BETWEEN SITE OF CUTANEOUS MELANOMA AND OCCUPATIONAL EXPOSURE TO SUNLIGHT}

\author{
T. Burke, S. Potter, D. Joyce, J. McCann, J. Kelly, A. Hussey, \\ P. Regan, M. Kerin
}

Department of Plastic, Reconstructive and Aesthetic Surgery, Department of General Surgery, University Hospital, Galway, Ireland

It is widely recognised that Melanoma is linked to sun exposure. Certain areas of the body are more chronically sun exposed, particularly the head and neck region. Certain occupations due to their nature result in greater exposure than others.

This retrospective study aims to compare clinical and prognostic factors of Melanomas at varying anatomical sites in order to detect differences, which may be associated to occupational sunlight exposure.

A retrospective analysis was carried out on 186 patients treated surgically for cutaneous Melanoma at University Hospital Galway. The records were examined and patients subdivided according to high occupational exposure (HOE) and limited occupational exposure (LOE). The clinical and histopathological data of those with HOE were then compared with that of patients with LOE. Univariate and multivariate analysis was applied to detect differences between $\mathrm{HOE}$ patients and LOE patients.

HOE accounted for $26.3 \%$ (49/186) of the cohort. The most common site of Melanoma for HOE patients was the head and neck (55\% 27/49) and the back for melanoma of LOE patients (36.5\% 50/ 137). The most common subtype is Lentigo Maligna in those with HOE and Superficial Spreading in those with LOE. HOE patients were older (mean age at diagnosis 63 vs. 51 years), predominantly 
male (92 vs. $34 \%$ ) and presented with slightly thicker tumours (1.8 vs. $1.72 \mathrm{~mm})$.

HOE Melanoma patients display distinct clinical and pathological features. Understanding the role of occupational exposure is vital for public awareness regarding sun protection.

Conflict of interest: None

Disclosures: None

\section{EFFECT OF PREVIOUS PERCUTANEOUS CORONARY INTERVENTION ON CARDIAC SURGERY-A SINGLE-CENTRE STUDY}

\author{
L. Sutton ${ }^{1}$, S. Early ${ }^{1}$, M. Tolan ${ }^{1}$, V. Young ${ }^{1}$, J. Moriarty ${ }^{2}$, \\ E. McGovern ${ }^{1}$ \\ Departments of Cardiac Surgery ${ }^{1}$ and Anaesthesia ${ }^{2}$, \\ St James's University Hospital
}

Introduction: The risk-factor profile of the cardiac surgery patient has changed radically in recent years, due to ever-increasing interventional cardiology procedures and patient factors, resulting in prolonged ICU length-of-stay and increasing pressure on finite ICU facilities.

Aim: Identification of patients who had a PCI prior to cardiac surgery and its effect on patient's peri-operative management and outcome; including operative intervention and post operative ICU length of stay. Methods: Prospective data entry of all patients undergoing cardiac surgery in a single centre between 2000 and 2008 was reviewed $(n=4,146)$. Data recorded included previous PCI, patient demographics, co-morbidities, procedure and post-operative complications. Results: $8 \%$ of cardiac surgery patients had previous PCI $(n=316)$. $9 \%$ of these patients were undergoing their second cardiac operation as opposed to $4 \%$ of patients with no previous PCI $(p<0.01)$. Patients with previous PCI were more likely to have hypercholesterolaemia, have unstable angina, and have an ejection fraction of 30 $49 \%$. These patients were also more likely to require emergency surgery and a pre-operative IABP.

Conclusions: While patients with previous PCI did not have a prolonged ICU stay they were at greater risk of a second operation, emergency surgery and the necessity for a pre-operative IABP. These patients exhibited significant pre-operative risk factors such as angina status CCS4 and a fair (30-49\%) ejection fraction. With cost effective efficient use of health care being a necessity for both clinician and policy-makers alike, the cost implications of these patients, requiring multiple operations, is an increasing point of concern.

Conflict of interest: None

Disclosures: None

\section{THE PREVALENCE OF PSA 'SELF-TESTING' AMONG CONSULTANT UROLOGISTS}

\author{
N.F. Davis, B.B. McGuire, H.D. Flood
}

Department of Urology, Mid-Western Regional Hospital, Limerick, Ireland

Introduction: Prostate specific antigen (PSA) has been used since 1986 as a biologic marker for prostate cancer. Despite widespread use among urologists, their opinion on PSA as a screening tool remains divided. Level-1 evidence demonstrating mortality benefits that outweigh the cost of overtreatment and overdiagnosis is lacking. This study aims to assess the prevalence of PSA 'self-testing' among Consultant Urologists
Methods: An anonymous online survey was emailed to 390 male consultant urologists worldwide with the following three questions:

1. Have you ever had your PSA checked?

2. Do you check your PSA annually?

3. How old are you?

One follow up email was sent to encourage non responders.

Results: Of the 390 male consultant urologists emailed 82 responded $(21 \%) .57 .3 \%(n=47)$ have not previously had their PSA tested and $42.7 \%(n=35)$ have previously had their PSA checked on at least one occasion, with $19.5 \%(n=16)$ of all respondents checking their PSA annually.

The mean age of respondents was 53.51 years and the prevalence of PSA self-testing was age related (Table 1).

Table 1 Prevalence of 'PSA self-testing' among Consultant Urologists according to age

\begin{tabular}{lllll}
\hline Age & $<50$ & $51-60$ & $61-65$ & $>65$ \\
\hline Percentage & $24 \%$ & $42.2 \%$ & $52.6 \%$ & $100 \%$ \\
Number & $6 / 25$ & $17 / 36$ & $10 / 19$ & $2 / 2$ \\
\hline
\end{tabular}

Conclusion: This study demonstrates the reservations among Consultant Urologists regarding PSA as a screening tool for prostate cancer. This is emphasized by the fact that only $42.7 \%$ (35/82) of male urologists surveyed (mean age, 53 years) have had their PSA tested with only $19.5 \%(16 / 82)$ checking their PSA annually.

Conflict of interest: None

Disclosures: None

\section{DAY-CASE LAPOROSCOPIC SURGERY: HOW CAN WE IMPROVE?}

E. Ibrahim, M. Salama, A. Lockley, M. Nolan, N. Kinsella, I. Ahmed Our Lady's Hospital, Navan, Co. Meath

Introduction: The Irish Health Service Reform (DOH\&C 2003) focuses on financial, professional and organizational accountability and responsibility. Utilizing more cost effective Day surgery therefore makes for prudent financial management. In the USA and Canada, day surgery accounts for nearly $90 \%$ of all surgery performed (Castoro et al. 2007) however in Ireland fewer than $30 \%$ of procedures are day cases (PA Consulting Group 2007). A review of the systems and processes already in place is essential.

Aim: To determine the efficiency of our day case laparoscopic surgery and identify areas for improvement.

Methods: A retrospective review of the records of 969 patients who underwent elective laparoscopic surgery during the last 5 years within our institute, to identify the percentage performed as day cases. These procedures included: Laparoscopic inguinal hernia repair (97 Pts.), laparoscopic cholecystectomy (30 Pts), diagnostic Laparoscopy (1 Pt) and gynaecological laparoscopic procedures (137 Pts). The review would focus on the outcome, safety and cost effectiveness, in addition to the rate of readmission within 2 days, and within 5 days. We could then compare with current standards.

Results: Of the 969 elective laparoscopic procedures performed, 265 (27.3\%) were day cases. Among those, 241 Pts. (91\%) were discharged home the same day, and 24 Pts (9\%) were admitted over night. Admissions included 4 Pts post hernia repair, 6 Pts post lap cholecystectomy and 14 Pts post lap gynaecological procedures. The principal causes for over night admission were: pain $37.5 \%$, minor 
haemorrhage $20 \%$, nausea $12.5 \%$ and urinary retention $8.3 \%$. The rate of readmission was $0.7 \%$ within 5 days (1 Pt. post lap. hernia admitted at day 2 for urinary retention and 1 Pt. post lap. cholecystectomy at day 4 for port-site infection). The cost of a day case versus overnight stay is EU.362 versus EU.964.14.

Conclusion: Day surgery is the gold standard approach for elective laparoscopic procedures, and should be considered the norm. Funding should be secured and redistribution of existing fund should be considered to extend Day Unit hours, and improve training and staffing levels. This will increase day case services and produce long-term cost benefits to both our Health Service and those it serves.

Conflict of interest: None

Disclosures: None

\section{SURGICAL SERVICES, A VICTIM OF CRIMINAL MINDS}

\author{
J.G. Hogan, M. McCafferty, P. Keeling \\ James Connolly Memorial Hospital, Blanchardstown, Dublin 15, \\ Ireland
}

Introduction: Surgical admissions as a result of crime related trauma is a significant burden on hospital expenditure. These patients are particularly costly requiring prolonged hospital stays, operative procedures and multidisciplinary input. Consequences of these costs are seen in all surgical departments-day ward closure, loss of theatre time, cancellations of elective procedures and overcrowded clinics Aims:

(1) Document the number of crime related injuries in 1 year.

(2) Describe the degree of violence and types in injuries encountered.

(3) Calculate the cumulative number of inpatient days and total cost.

Methods: 102 cases from 01/01/2008 to 31/12/2008 were reviewed on an individual basis. The type of crime, weapons used, resulting injuries and operative procedures performed were documented. We also recorded age, gender, nationality, length of stay, private or public, number requiring theatre and other indeterminate costs (number of CTs, ambulance call outs, OPD review). We estimate a cumulative cost based on the hospitals billing policy.

Results: 102 admissions in 2008, with decreasing numbers through 2006 (200 admissions), 2007 (180 admissions) and 2008. 83\% were male the majority in their thirties. Average cost per patient per day was 970 euro, with a total of 308 inpatient days totalling a cost of 299,000 euro. Weapons encountered include guns, knives, pitch forks, knuckledusters etc. Procedures performed include laparotomy, thoracotomy, lung and liver lobectomies.

Conclusion: Crime continues to burden the hospital service. It contributes significantly to loss of services and the magnitude of the problem will only rise as the economy declines.

Conflict of interest: None

Disclosures: None

\section{COMPARING CLINICIAN AND HIPE BASED DISCHARGE SUMMARY CODING AND ITS IMPACT ON FUNDING WITHIN A UNIVERSITY TEACHING HOSPITAL SURGICAL DEPARTMENT}

R. Murphy, T. McVeigh, P. Waters, G.T. O’Donoghue, M. Kerin
Department of Endocrine and Breast Surgery, University College Hospital Galway, Newcastle Road, Galway, Ireland

Introduction: Hospital in-patient enquiry (HIPE) uses medical notes and discharge summaries to collect inpatient clinical and epidemiological data. The economic social and research institute allocates hospital budgets for service planning according to this data 1 .

The aim of this study was to retrospectively evaluate discharge summaries to maximise coding accuracy and investigate budgetary shortfalls.

Methods: A retrospective study was carried out on 100 consecutive Breast and Vascular Surgery UCHG Electronic Discharge Summaries.

Primary and secondary diagnosis, procedures performed and length of stay were re-coded by clinical personnel and funding totals re-calculated.

Results: No difference in primary diagnosis was noted, whereas a $25.8 \%$ increase in Breast secondary diagnosis and a $67.3 \%$ increase in Vascular secondary diagnosis following clinician EDS recoding was recorded.

$62 \%$ of EDS had some diagnosis inaccuracy and a $48 \%$ inaccuracy in procedure date/length of stay was noted.

These led to a total vascular funding shortfall of $€ 231,102$.

Discussion: In times of economic scrutiny it is imperative to exploit patient discharge summary accuracy. The absence of formalised discharge summary training for clinicians identifies an embarrassing lack of financial awareness which if not rectified will continue to adversely affect resource allocation.

References:

(1) Wiley M et al. Irish Journal of Medical Science, Vol. 174, No. 2, June 2005

Conflict of interest: None

Disclosures: None

\section{ASSESSMENT OF SUITABILITY OF RECTAL CANCER FOR A LAPAROSCOPIC APPROACH-A SINGLE CENTRE EXPERIENCE OF 122 PATIENTS}

D.W. Good, J.M. O’Riordan, D. Moran, F.B.V. Keane, E. Eguare, D.S. O'Riordain, P. Neary

Department of Colorectal surgery, AMNCH, Tallaght, Dublin 24, Ireland

Introduction: Debate persists regarding the feasibility of laparoscopic resection for rectal cancer because of the long learning curve and questionable oncological safety.

Aim: Determine the short-term outcomes of patients undergoing laparoscopic rectal cancer surgery.

Methods: Patients undergoing surgery for rectal cancer between June 2005 and September 2009 were reviewed using a prospective colorectal cancer database. Rectal cancer was defined as a tumour with a lower margin at

Results: One hundred and twenty-two patients underwent surgery for rectal cancer during this time. One hundred and ten patients had laparoscopic resection, five of these were converted to open (conversion rate $4.5 \%$ ) and twelve had planned open. One hundred and five patients had an anterior resection. Median node retrieval was 10 (7-14) for the laparoscopic group and 12 (9-12) for the open group ( $p=n s)$. Median length of stay was 10 days (range 6-14) for the laparoscopic group and 20 days (range 10-24) in the open group $(p=0.03)$. The positive circumferential margin rate was $3.3 \%$ in the laparoscopic group compared with $5.9 \%$ in the open group. The wound infection rate was $3 \%$ with 
laparoscopy and $18 \%$ with open surgery $(p=0.04)$. The clinical anastomotic leak rate for the laparoscopic group was $4.8 \%$.

Conclusions: $86 \%$ of rectal cancers can undergo a laparoscopic resection with decreased hospital stay, wound infection rate and equivalent oncological outcomes compared with open surgery. Laparoscopic surgery is the standard of care for rectal cancer in our institution. Conflict of interest: None

Disclosures: None

\section{CHYLOTHORAX AND CONGENITAL CARDIAC SURGERY; A SINGLE CENTRE STUDY OF 452 PAEDIATRIC PATIENTS}

S. Halpenny, S. Early, C. McMahon, A.E. Wood, M.J. Redmond, D. Mannion, L. Nolke

Departments of Surgery and Anaesthesia, Our Lady's Hospital for Sick Children, Crumlin, Dublin, Ireland

Introduction: Chylothorax is a significant and potentially lifethreatening complication that can arise after cardiac surgery for congenital cardiac defects. Treatment strategies include nutritional modification, somatostatin analogues, total parentral nutrition (TPN) and surgical intervention. Controversy exists regarding the most effective treatment. The aim of this study was to investigate the incidence of post operative chylothorax and to examine the success rate of current therapeutic interventions in a paediatric population post cardiac surgery.

Methods: All children who underwent cardiac surgery in 2008 were enrolled in the study. Data was collected on patients who developed a chylothorax. Data recorded included patient age, gender, weight and age at operation, congenital syndromes, cardiac anomaly, cardiac operation performed (including thymectomy), left internal jugular line(LIJL)placement, chest drain insertion, length of ICU stay, total length of stay and treatment instigated for chylothorax

Results: 462 cardiac surgery procedures were performed in 2008. 59 patients developed post-operative chylothorax $(12.8 \%)$. The majority of these patients had repair of atrioventricular septal defect (AVSD) (48\%), all of whom had Trisomy $21.68 \%$ of children had a LIJL. Treatment was successful with nutritional modification, somatostatin and/or TPN therapy in all but one case which required bethadine pleurodesis. No patient required thoracic duct ligation.

Conclusion: These data demonstrate the effectiveness of nutritional modification combined with somatostatin analogues and TPN and that the requirement for surgical intervention is extremely rare in this patient cohort. These data also highlight several interesting results associated with the development of chylothorax in a paediatric population which warrant further study.

Conflict of interest: None

Disclosures: None

\section{INVESTIGATION OF POTENTIAL ROLE OF CCL5 IN BREAST CANCER PROGRESSION}

\author{
M. C. Hartmann, R. M. Dwyer, M. Costello, M. J. Kerin \\ Department of Surgery, National University of Ireland, Galway, \\ Ireland
}

CCL5/RANTES has a role in inflammation and has been implicated in breast cancer progression. The aim of this study was to determine circulating and tissue levels of CCL5 in breast cancer patients, and investigate a potential relationship with TGF $\beta 1$. Circulating CCL5 was quantified using ELISA in breast cancer patients $(n=110)$ and controls $(n=69)$. Tumour tissue samples $(n=44)$ were available on a subset of these patients and reduction mammoplasty tissues served as controls $(n=16)$. RQ PCR was carried out targeting CCL5, CCR5, TGF $\beta 1$ and TGF $\beta$ Receptor II (TGF $\beta$ RII). Within the breast cancer cohort, patients with Basal subtype tumours had significantly higher circulating CCL5 $(77.72 \pm 20.52 \mathrm{ng} / \mathrm{ml})$ than Luminal A subtype $(47.85 \pm 3.77 \mathrm{ng} / \mathrm{ml}, p<0.01)$. Patients with node negative disease had higher levels of CCL5 $(61.49 \pm 7.48 \mathrm{ng} /$ $\mathrm{ml})$ than those with $\leq 2$ lymph nodes positive $(40.96 \pm 6.34 \mathrm{ng} / \mathrm{ml}$, $p<0.05$ ). As the number of positive nodes increased, CCL5 increased and was significantly higher in patients with $\geq 10$ nodes positive $(p<0.05)$. This trend has previously been associated with circulating TGF $\beta 1$, and further investigation revealed a positive correlation between circulating CCL5 and TGF $\beta 1 \quad(n=138$, $r=0.44, p<0.0001)$. Expression of CCL5 and CCR5 in tumour tissue was significantly higher than normal tissue $(p<0.05)$, with a positive correlation observed between the ligand and its receptor ( $n=60, r=0.46, p<0.0001)$. TGF $\beta 1$ expression was significantly higher in tumour compared to normal tissue $(p<0.001)$. The data presented supports a role for CCL5 in breast cancer, particularly in relation to nodal status. Further investigation of a link with TGF $\beta 1$ is warranted.

Conflict of interest: None

Disclosures: None

\section{PATTERNS OF DISEASE RECURRENCE IN AN IRISH BREAST CANCER COHORT}

\author{
S. Lewis, D.P. McCartan, Z.M. AlHilli, A.D.K. Hill \\ Department of Surgery, Beaumont Hospital, Dublin 9, Ireland
}

Data from the National Cancer Registry has shown improved 5 year survival rates in breast cancer patients treated between 2000 and 2004. However, distant metastases remain the main cause of death in patients with breast cancer. The objective of this study was to examine patterns of disease recurrence in all patients treated for early breast cancer over a 10 year period at Beaumont hospital.

Data was obtained from a retrospective database for all patients who underwent surgery for breast cancer from 1996 to 2006 inclusive. Patients with metastatic disease at presentation were excluded. Median length of follow up in those without disease recurrence was 5.4 years. Tumours were categorised according to molecular subtype. Recurrences were classified as local or systemic events.

From 625 patients in total, $186(30 \%)$ recurrence events were recorded at a median time of 2.6 years from diagnosis. Rates of recurrence were highest and time to disease recurrence were shortest in the basal (44\%, 2.2 years) and Erbb2 (40\%, 2.4 years) subgroups with the lowest recurrence rates and longest disease free survival seen in the luminal A (22\%, 4.1 years) group ( $p<0.001$ for $\chi^{2}$ recurrence rate and ANOVA for time to recurrence). Bone was the most commonly identified site of first distant metastases $(39 \%)$ followed by lung (29\%).

The prognostic impact of the molecular subtypes of breast cancer is evident in this Irish cohort. In an era of enhanced therapeutic options, new molecular insights will further enhance our understanding of breast cancer metastasis.

Conflict of interest: None

Disclosures: None 


\section{COLONIC INTERPOSITION: THE BEAUMONT EXPERIENCE}

\author{
J.C. Bolger, E. Myers, P. Broe \\ Department of Surgery, Beaumont Hospital, Beaumont, Dublin 9, \\ Ireland
}

Introduction: Oesophagectomy with lymphadenectomy remains the optimal therapeutic option in resectable oesophageal cancer, and furthermore has a role to play in oesophageal failure secondary to benign oesophageal disease [1]. The preferred primary conduit for replacement is a gastric tube. We reserve colonic interposition for reconstruction following primary conduit failure [2], or where the stomach is unsuitable for use as a conduit.

Methods: 270 patients underwent oesophagectomy in Beaumont between 2001 and 2009, of whom 17 underwent colonic interpositions. We reviewed their sex, age, length of stay, histology, indication for colonic interposition, complication rate and long-term outcome. Results: 17 patients ( 13 male, 4 female). Mean age was 58.36 years, median 59 (24-74). Mean length of stay was 65.23 days, median 32 (10-176). Indications were primary graft necrosis (8, i.e $3 \%$ of total); oesophageal dysmotility (3); primary gastric tumour with oesophageal extension (3); positive resection margins (1); oesophageal tumour with gastric extension (1); broncho-oesophageal fistula (1). Histologically: 12 adenocarcinoma; 1 signet cell carcinoma; one NSCLC and three benign disease. Wound infections occurred in six patients. Anastomotic leak occured in two patients, both proximal anastomosis. Respiratory sepsis occurred in seven patients. One developed neutropaenic sepsis, and one developed an abdominal collection. Three patients developed late anastomotic stricture, all at the colo-gastric anastomosis. Eight patients are currently alive and well. Seven died from late disease recurrence, while two died post-operatively from complications.

Conclusions: Colonic interposition can be performed both for primary conduit failure following oesophagectomy, and as primary treatment for benign oesophageal disease with acceptable morbidity and mortality.

References:

(1) Orringer MB, Marshall B, Chang AC, Lee J, Pickens A, Lau CL (2007) Two thousand transhiatal esophagectomies: changing trends, lessons learned. Ann Surg 246:363-374

(2) Wormuth JK, Heitmiller RF (2006) Esophageal conduit necrosis. Thorac Surg Clin 16(1):11-22

Conflict of interest: None

Declarations: None

\section{REVIEW OF APR IN BEAUMONT HOSPITAL 1996-2006: BENIGN DISEASE REPRESENTS AN IMPORTANT COMPONENT OF OPERATIVE EXPERIENCE}

D.R. Gough, J. Keogh, A. Hanly, N. McCawley, J. Deasy, D.A. McNamara

Department of Colorectal Surgery, Beaumont Hospital, Dublin, Ireland

Abdominoperineal resection (APR) is a technically challenging operation. The pelvic component of the operation is identical to that of total mesorectal excision, with its well-defined planes, however the perineal dissection requires detailed anatomic knowledge to correctly achieve a quality resection. Poor results of APR for cancer are widely reported prompting us to review our experience of this operation.

All patients treated with APR between 1996 and $2006(n=85)$ were reviewed. There were 63 resections for cancer and 22 for benign disease, 14 of which were for IBD. Of the 63 cancer resections, 57 were for primary adenocarcinoma, 4 were for recurrent rectal adenocarcinoma and 2 were for squamous cell carcinoma. $76 \%$ were curative resections. Most non-curative resections had metastatic disease at time of surgery. Over $80 \%$ received either neoadjuvant or adjuvant radiotherapy. Operative margins, including the circumferential margin were negative in 54 patients $(86 \%)$. Overall 3 year survival rates for curative and salvage APRs were 65 and 27\%, respectively.

Good results from APR can be achieved in a specialist unit. Abdominoperineal resections for benign disease represents a substantial component of operative experience in performing this technically difficult procedure. Separation of benign cases that warrant complex pelvic surgery from pelvic cancer surgery risks reducing the skill levels of surgeons treating cancer and cannot be supported. Conflict of interest: None Disclosures: None

\section{A "NATURAL KILLER" ROLE FOR THE OMENTUM IN TUMOUR IMMUNITY}

\author{
D.B. O' Connor ${ }^{1}$, D. O' $\mathrm{Shea}^{2}$, C. O'Farrelly ${ }^{3}$, L. Lynch $^{2,4}$, \\ D.C. Winter ${ }^{1}$
}

Surgery ${ }^{I}$, St. Vincent's University Hospital, Elm Park Dublin 4, Ireland, Obesity and Immunology ${ }^{2}$, St. Vincent's University Hospital, Dublin 4, Ireland, Trinity College ${ }^{3}$, Dublin 2, Ireland, Harvard Medical School ${ }^{4}$, Boston, USA

Tumour-infiltrating cytotoxic $\mathrm{T}$ cells are critical for an effective antitumour immune response to cancer. The greater omentum has been identified as a site of specific T lymphocyte subset accumulation

This study aimed to determine if the immune repertoire and antitumour properties of the omentum is distinct from other adipose tissues.

Omental samples from patients with colorectal cancer and age/sex matched controls were analysed by flow cytometry to characterise lymphocyte populations. Samples from subcutaneous, mesenteric and pericolic fat and blood were similarly examined. INKT (invariant natural killer $\mathrm{T}$ cells) and Natural Killer cells (CD3 + NK) were isolated from omentum and blood by magnetic bead separation and their cytotoxicity against autologous cancer cells was measured using a flow cytometric based assay. Data is presented as mean and differences between groups were assessed using Mann-Whitney test.

The omentum had a higher concentration of lymphocytes than other adipose tissue. (Omentum: $24.6 \times 103 / \mathrm{g}$, Mesenteric: $12.7 \times 103 / \mathrm{g}$, Pericolic: $4.7 \times 103 / \mathrm{g}$, Subcut: $8.73 \times 103 / \mathrm{g}$ ). In particular, iNKT and CD3 + NK subtypes were found at 14 -fold and fivefold higher concentrations respectively, in omentum than other fat depots and at much higher concentrations compared to peripheral blood (100-fold and 20fold, respectively). Omental iNKT $(p=0.004)$ and CD3 + NK ( $p=0.001)$, but not other T cells, were significantly depleted in colorectal cancer patients $(n=8)$ versus controls $(n=8)$. Both killed autologous tumour cells, but not uninvolved colon.

Distinct cytotoxic immune cells accumulate in the omentum but are specifically depleted in colorectal cancer and may play an important role in tumour immunity.

Conflict of interest: None

Disclosures: None 


\section{WHY ROUTINE ICU ADMISSION AFTER ELECTIVE OPEN INFRARENAL ABDOMINAL AORTIC ANEURYSM (AAA) REPAIR IS NO LONGER EVIDENCE BASED PRACTICE}

\author{
D. Ryan, G. McGreal
}

Department of Vascular Surgery, Mercy University Hospital, Grenville Place, Cork, Ireland

Background: Elective Open Infrarenal Abdominal Aortic Aneurysm (AAA) repair is major surgery performed on high risk patients. Routine ICU admission postoperatively is the current accepted standard of care in Ireland. Evidence based medicine has shown that the majority of elective infrarenal AAA patients can be safely admitted to a high dependency unit (HDU) for postoperative management, sparing valuable and costly ICU resources [1]

Aim: To determine, 'could the high risk patients that subsequently necessitated ICU admission postoperatively have been reliably identified preoperatively?'

Methods: A retrospective analysis of all elective open infrarenal AAA repairs in our institution over a 3 year period was performed. The Estimation of Physiological and Surgical Stress (E-PASS) model was used as our risk stratification tool for predicting postoperative morbidity. Renal function was also considered as a predictor of outcome, independent of the E-PASS

Results: $80 \%(n=16)$ were admitted to ICU. Only $30 \%(n=6)$ of the total study population necessitated intensive care. There were nine complications in seven patients in our study. The E-PASS comprehensive risk score (CRS)/Surgical stress score (SSS) were found to be significantly associated with the presence of a complication $(p=0.009) /(p=0.032)$ respectively. Serum creatinine $(p=0.013)$ was similarly significantly associated with the presence of a complication.

Conclusions: The E-PASS model possessing increasing external validity is an effective risk stratification tool in safely deciding the appropriate level of postoperative care for elective infrarenal AAA repairs.

(1) Lawlor DK, Lovell MB, DeRose G, Forbes TL, Harris KA (2004) Is intensive care necessary after elective abdominal aortic aneurysm repair? Can J Surg 47(5):359-363.

Conflict of interest: None

Disclosures: None

\section{SURGICAL PROCEDURES AND THE INTERNET. THE CHALLENGE OF ENSURING PATIENTS ARE ACCURATELY INFORMED}

\author{
E.K. Walsh, F. Hand, J.O. Murphy, M.R. Kell \\ Eccles Breast Screening Unit, Mater Misericordiae University \\ Hospital, BreastCheck, Eccles Street, Dublin 7, Ireland
}

\begin{abstract}
Aims: Breast augmentation is one of the most commonly performed aesthetic surgical procedures. For optimal surgical outcome, patients need to be fully informed of the procedure and have realistic short and long-term expectations. The Internet is a major source of this information; however it is unregulated and often misleading. We aimed to assess the quality of information obtained by patients using the foremost search engines and search terms.
\end{abstract}

Methods: Using the three leading search engines Google, Yahoo and Bing, we searched for the most popular relevant terms; "breast augmentation", "breast enlargement", "breast implants" and "breast enhancement". We scored the accuracy of the information obtained from the top 30 ranked websites for each search. Websites were scored for their description of the procedure, postoperative complications and long-term issues based on the Medicines and Healthcare products Regulatory Agency (MHRA) patient information leaflet.

Results: 183 unique websites were identified and scored out of 68 . Websites found using Google scored higher than Bing or Yahoo (mean score 22.0 vs. 19.7 and 18.6, $p<0.01$ ). Searching for the term "breast augmentation" or "breast implants" produced higher scoring websites than "breast enlargement" or "breast enhancement" (mean scores 28.0 and 27.6 vs. 14.2 and 10.7, $p<0.01$ ). There was poor correlation between website ranking by search engines and website's score $\left(R^{2}=0.011\right)$. Seven websites contained incorrect information and 55 were irrelevant, scoring 0 out of 68 .

Conclusions: Ensuring our patients are well informed is a challenge for surgeons. Comprehensive information on surgical procedures can be found on the internet. However, this can be difficult to locate, even using the most popular search terms and leading search engines. Directing patients to better-quality websites may enhance their understanding prior to surgery and improve satisfaction.

Conflict of interest: None

Disclosures: None

\section{RADIATION SAFETY AWARENESS... OR NOT?}

M. Murphy, Z. Martin, G.C. O’Brien, N. Cloete, M.P. Colgan, S.N. Haider, P. Madhavan, S.M. O’Neill, D.J. Moore

Department of Vascular and Endovascular Surgery, St James's Hospital, Dublin 8, Ireland

Investigations involving ionising radiation are an accepted and fundamental part of medical practice in spite of the small but definite risk to patients' health. Despite advances in imaging technology, education provision in the field of radiation safety is scarce.

We compiled a questionnaire containing 30 questions relating to the doses of radiation associated with some of the more commonly ordered radiological investigations and also general radiation safety awareness. Hospital doctors were randomly asked to fill out the questionnaire without prior notice. Correct answers to the questions were obtained from the European Commission's guidelines for Radiation Protection 118.

100 doctors completed the questionnaire. Nobody answered all the questions correctly. The average number of correct answers was 16 (54\%) and this was only slightly better in those who had completed a radiation protection course $18(58 \%)$.

\begin{tabular}{lll}
\hline & $n$ & $\%$ Correct answers \\
\hline Consultants & 16 & 61.57 \\
Registrars & 32 & 56.45 \\
SHO's & 23 & 51.59 \\
Interns & 29 & 53.1 \\
\hline
\end{tabular}

Three percent and $22 \%$ respectively thought that ultrasound and MRI exposed patients to ionising radiation. Twenty-one percent did not know that PET exposes patients to ionising radiation and $42 \%$ underestimated the actual radiation exposure. $60 \%$ underestimated the radiation exposure for a $\mathrm{CT}$ abdomen and pelvis. Only $10 \%$ of people 
knew that the 3 most important factors in reducing radiation exposure were time, distance and shielding.

There is a lack of awareness of the degree of exposure to ionising radiation for common investigations at all grades. Education in radiation safety awareness should be mandatory for all clinicians who are permitted to request investigations, which expose patients to ionising radiation.

Conflict of interest: None

Disclosures: None

\section{THE USE OF SEQUENTIAL COMPRESSION BIOMECHANICAL DEVICE IN CRITICAL LIMB ISCHEMIA PATIENTS WITH UN-RE- CONSTRUCTABLE PERIPHERAL VASCULAR DISEASE. A 5 YEARS EXPERIENCE IN A TERTIARY REFERRAL VASCULAR CENTRE}

\author{
N. Hamada, S. Sultan \\ University College Hospital Galway (UCHG), Western Vascular \\ Institute (WVI), Newcastle, Galway, Ireland
}

The aim of our study is to find out the long term outcome of the use of the SCBD as an alternative treatment for patients with CLI who are unfit for revascularisation or due to severe co-morbidity scores and expected poor intervention outcome.

Patients were assessed on an intention to treat basis. Compositeprimary endpoints were limb-salvage, sustained clinical improvement, rest-pain resolution, and 90-day mortality. Second endpoints were hemodynamic outcomes with increase in popliteal artery flow and toe pressure, ulcer healing, QTWIST, Qualy and costeffectiveness.
From 2004 to 2009 we reviewed more than 4500 patients with PVD. 707 of them presented with CLI, 518 had one or more intervention for their PVD, while 189 were not candidates for surgery. Only 170 patients had joined the SCBD programme for 3 months at $3 \mathrm{~h}$ bi-daily. We matched controlled 75 primary amputations.

Demographic data was recorded according to the International Classification of Disease code. Imaging was primarily by Duplex Ultrasound Arterial Mapping.

Sustained clinical improvement was reported in $68 \%$ of patients. Sustained hemodynamic improvement were noted with; Mean toe pressure increase from 39.9 to $55.42 \mathrm{mmHg}$ post 12 months of treatment with mean difference in Toe Pressure of $15.49 \mathrm{mmHg}$ (95\%CI $=8.06-22.92) P=0.0001$

30 day mortality was $99.4 \%$. Mean Amputation free survival rate was 18 months with limb salvage rate at 5 years of $94 \%$. Freedom from MACE at 5 years was $62.5 \%$. All cause survival was $68.4 \%$ at 5 years.

The primary amputation rate has decreased by One-Third From $33 \%$ in the Period 1989-2000, to $21 \%$ since introduction of subintimal angioplasty in 2001 and to less than $6 \%$ per year since patients were applied for SCBD in 2004.

We managed 170 SCBD patients at one-fourth of the cost of 75 primary amputations in 62 patients with enhanced QUALY and superior QTWiST.

SCBD has superior limb salvage, ameliorated amputation free survival, reduced length of hospital stay and provided rapid relief of rest pain without any intervention in patients with limited life expectancy. SCBD is a valuable tool in the armamentarium of the vascular physicians dealing with CLI with Unreconstructable PVD and improve the quality of life at a community level.

Conflict of interest: None

Disclosures: None 